DEPARTMENT OF THE INTERIOR

U.S. GEOLOGICAL SURVEY

\title{
POROSITY, DEPTH, AND THERMAL-MATURITY DATA \\ FOR SANDSTONES OF THE ANADARKO BASIN, OKLAHOMA, AND OTHER SELECTED LOCATIONS IN THE NORTHERN HEMISPHERE
}

by Timothy C. Hester ${ }^{1}$ and James W. Schmoker ${ }^{2}$

Open-File Report 93-230

This report is preliminary and has not been reviewed for conformity with U.S. Geological Survey editorial standards and stratigraphic nomenclature. Any use of trade, product, or firm names is for descriptive purposes only and does not imply endorsement by the U.S. Government.

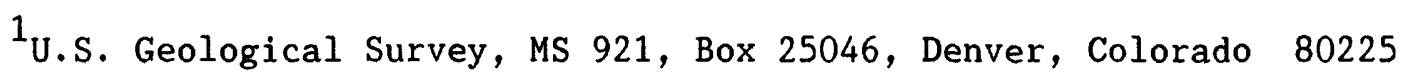

2U.S. Geological Survey, MS 960, Box 25046, Denver, Colorado 80225 
Introduction and Description of Tables................ 1

Table 1A Description of Box-Diagram Porosity Data Set....... 7

Table 1B Box-Diagram Porosity Percentiles............... 13

Table 2A List of Anadarko Basin Wells from which Log Porosity Data Were Obtained..................... 22

Table 2B Anadarko Basin Well-Log Porosity Data............ 23

Table 3A List of Anadarko Basin 0il and (or) Gas Reservoirs from which Hydrocarbon-Reservoir Porosity Data Were obtained........................... 36

Table 3B Anadarko Basin Hydrocarbon-Reservoir Porosity Data... 40 References

General............................ 42

Box-Diagram Porosity Data Set (Tables $1 A$ and 1B).... 42 Anadarko Basin Hydrocarbon-Reservoir Porosity Data Set (Tables $3 A$ and $3 B$ ) ................... 45

Interpretive Papers Based on the Data of this Report. 46 


\section{INTRODUCTION}

This report is a compilation of sandstone-porosity, depth, and thermal maturity $\left(R_{0}\right)$ data collected by the authors over several years, from many published and unpublished sources. Additional information, such as data locations and source, age of unit sampled, and references, is supplied where applicable. For well data, the operator, lease, field, and reservoir names, and formation thicknesses are also included.

The porosity data are from core-plug measurements, well logs, or thin-section point counts. $R$ data are averages of direct measurements or estimated values using empirical $R$-depth curves. These data represent a comprehensive sampling of sandstones of diverse ages, geologic settings, diagenetic facies, and thermal histories, from numerous basins in the Northern Hemisphere (fig. 1). Thus, the porosity-R data presented here provide a preliminary reference framework of sandstones in general with which to compare local sandstone porosity data or to predict regional trends. These data are the basis for the interpretive papers cited in this report.

The data presented here are grouped into sets 1,2 , and 3 ; each set consists of tables $A$ and $B$. The "A" tables locate, describe, and (or) identify the numerical data of the "B" tables, by using a cross reference key found in the first column of each table. In regard to size, format, geographic scope, and data sources and measurement methods, etc., the 3 data sets differ considerably. Although the data tables are for the most part self explanatory, several aspects warrant further attention. Therefore, each set is discussed individually in the following paragraphs.

\section{TABLES 1A AND 1B}

Table 1, the largest of the three data sets, represents sandstones from numerous basins in the Northern Hemisphere (fig.1) and many sources, both published and unpublished. The vast majority of individual porosity measurements (more than 8,000), from which the data of Table 1 are derived, are helium-porosimeter measurements of 1-inch core-plugs. Well-log and thin-section point-count data are also included, where noted.

The porosity data of Table 1 are not individual measurements, but are percentiles of a porosity distribution. Each porosity distribution is made from a group (suite) of individual measurements from a specific geologic interval and thermal-maturity setting. Each measurement suite is assigned a single $R_{0}$ value (usually the average of measured values for that suite), and is described in terms of "box-diagram percentiles" (fig. 2; Cleveland, 1985) and the single highest porosity measurement. The percentiles, when plotted in the form of a box-diagram histogram (fig. 2), graphically represent the porosity distribution.

The reason for this type of presentation is twofold. First, because the number of porosity measurements for each suite varies significantly, some suites would carry more statistical weight simply because they are represented by more measurements (Schmoker and Hester, 1990). Second, by reporting porosity measurements as a distribution rather than as a single 


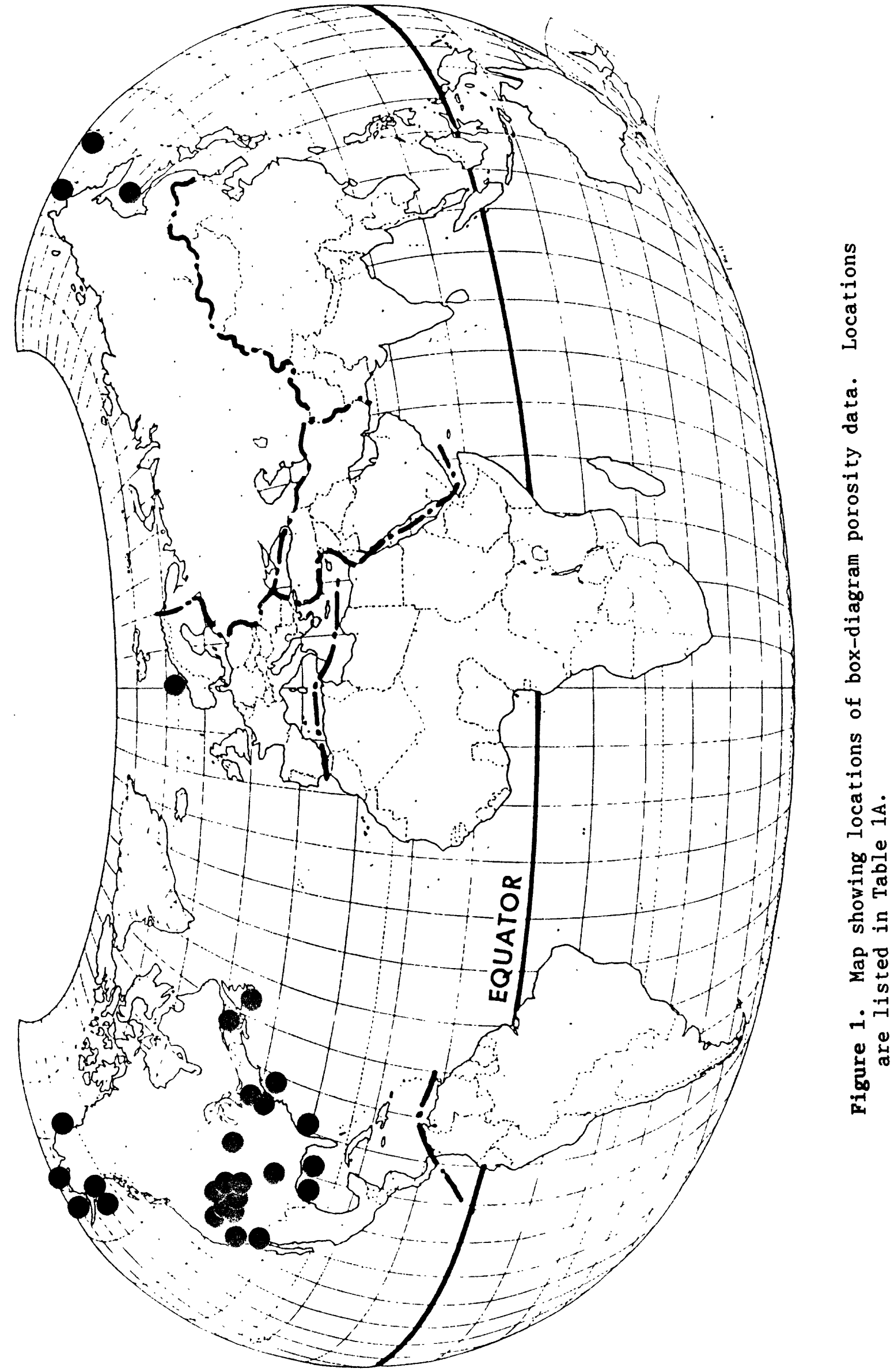



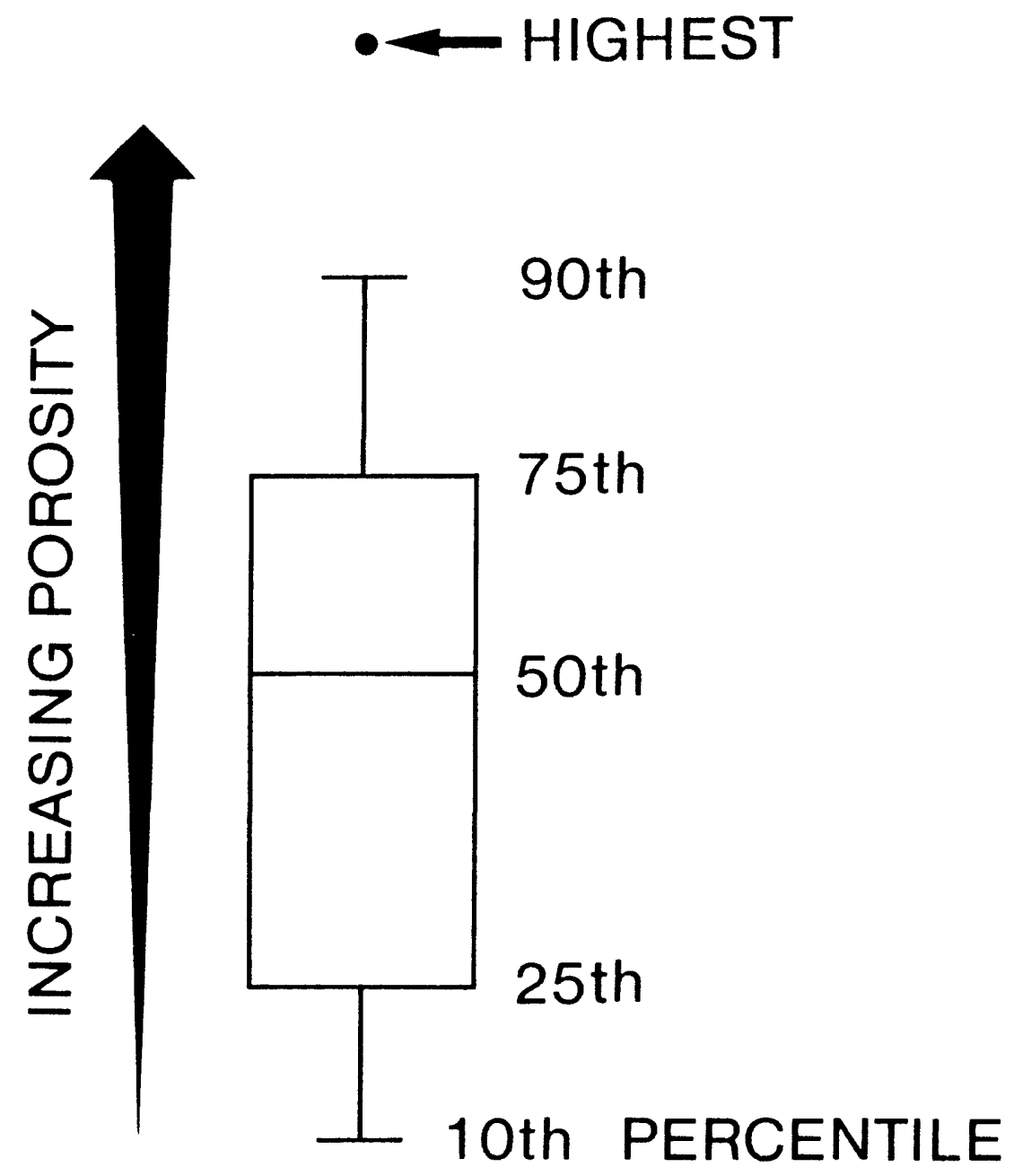

Figure 2. Box diagram (described by Cleveland 1985) illustrating data format of Table $1_{\text {th }}$ Porgsity distribution at each location is represented by $10^{\text {th }}, 25^{\mathrm{th}}, 50^{\mathrm{th}}, 75^{\mathrm{th}}$, and $90^{\mathrm{th}}$ porosity percentiles, and by single highest porosity measurement. 
value, much information about porosity variability is retained. Porosity trends can then be treated as a function of thermal maturity (or depth) while also displaying the variability of each measurement suite (Schmoker and Higley, 1991). The source of the data, and the age and location of the sampled interval(s) are given in Table $1 \mathrm{~A}$. The $10 \mathrm{th}, 25 \mathrm{th}, 50 \mathrm{th}$, $75 \mathrm{th}$, and $90 \mathrm{th}$, percentiles of the porosity distribution, the single highest porosity measurement, and the number of porosity measurements for each suite are given in Table 1B.

\section{TABLES 2A AND 2B}

Table 2 consists exclusively of well-log data from the central and southern Anadarko basin. These porosity data consist of more than 800 individual measurements representing more than 7,000 net $\mathrm{ft}$ of Paleozoic-age sandstone from 33 well locations in the central and southern Anadarko basin (fig. 3 ).

Sandstone is identified in each well, using compensated-neutron and formation-density logs run on limestone matrix, and is then subdivided into intervals of uniform log character. The neutron and density porosity of each interval ( 4 or more $\mathrm{ft}(1.2 \mathrm{~m}$ ) thick) is averaged (fig. 4 ), and its true porosity determined using standard neutron-density crossplots. To exclude shaley sandstones from the data set, the shift of true porosity toward the "shale point" of the neutron-density crossplot is allowed only 2 porosity units.

$R_{0}$ values are calculated for each sandstone interval using an empirical $R$-depth relationship developed for the Anadarko basin by Schmoker (1986). Well information is given in Table $2 \mathrm{~A}$; porosity, $\mathrm{R}_{0}$, depth, unit thickness, and age are given in Table 2B.

\section{TABLES 3A AND 3B}

Table 3 consists of porosity measurements of 105 Paleozoic-age sandstone oil and gas reservoirs of the Anadarko basin, Oklahoma (fig. 3). These data are from published oil- and gas-field compilations referenced in this report.

$R$ values are calculated as in the previous data set (Schmoker, 1986). Field and reservoir names, and approximate locations are given in Table $3 \mathrm{~A}$; porosity, depth, age, and $R_{0}$ are given in Table $3 B$. 


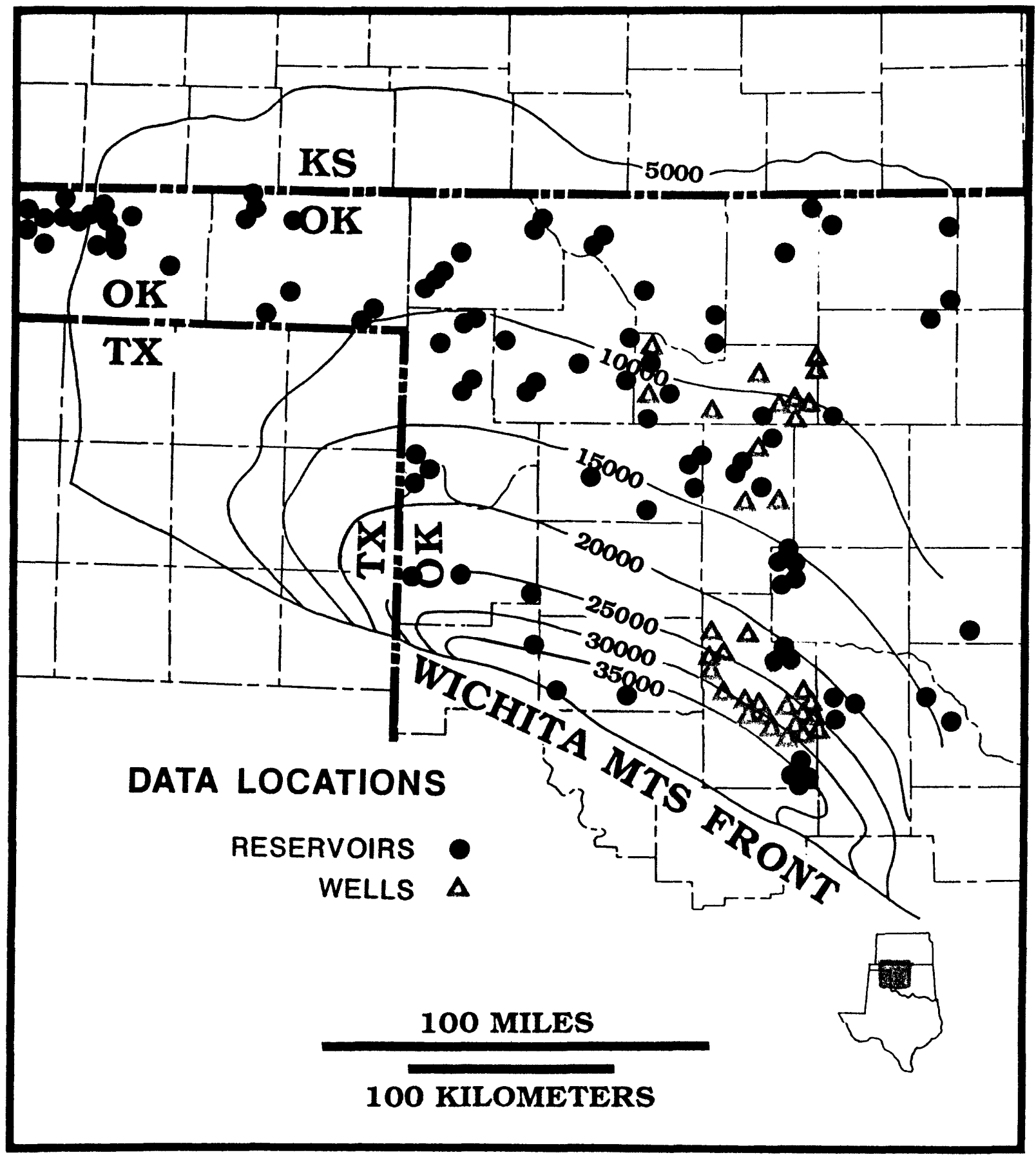

Figure 3. Map showing Anadarko basin total-sediment isopachs ( $f t$ ) and data locations. Well locations are listed in Table 2A. Reservoir locations are listed in Table $3 \mathrm{~A}$. 


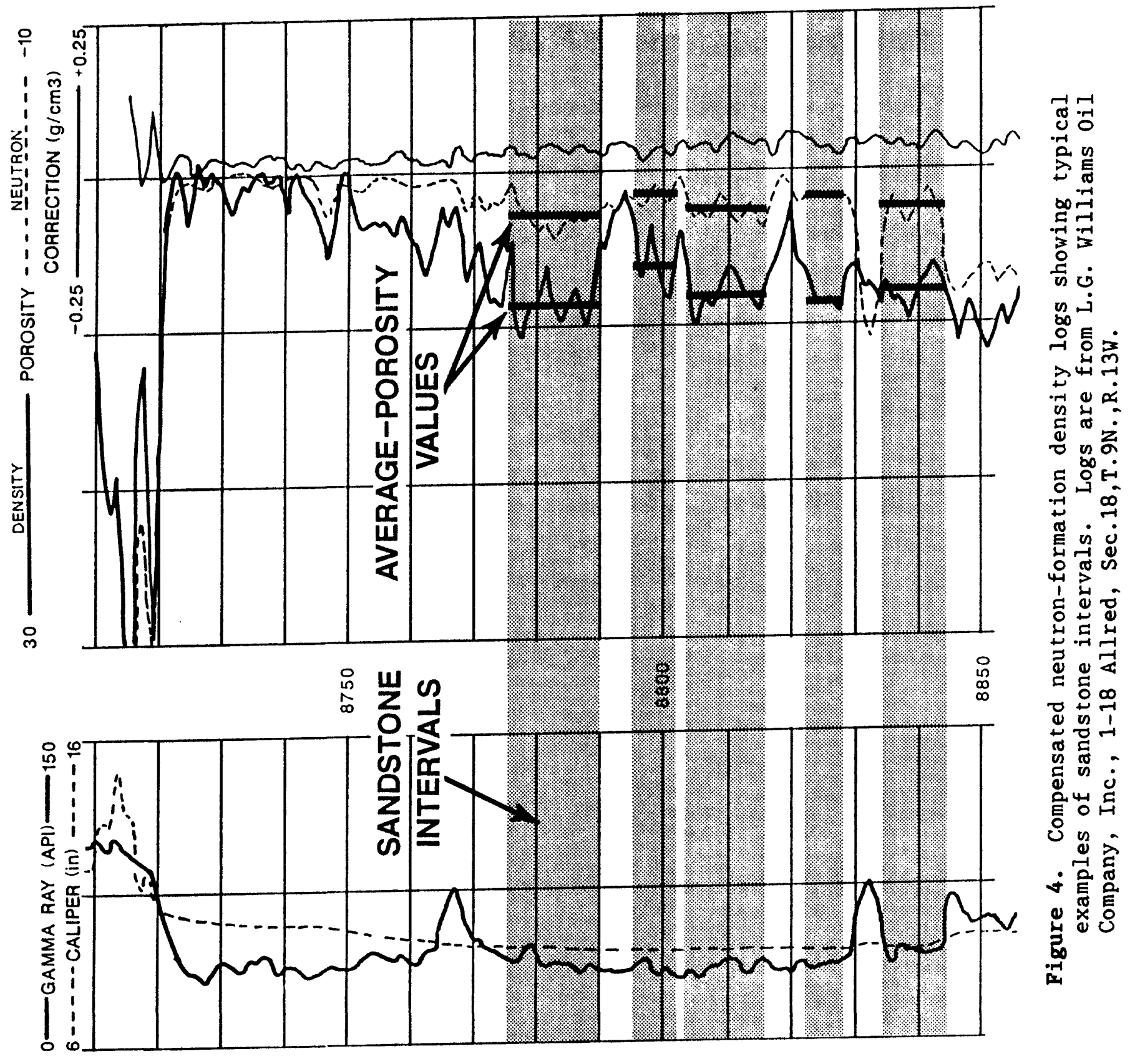


Table 1A. DESCRIPTION OF BOX-DIAGRAM POROSITY DATA SET

[ ${ }^{1}$ Data-identification number refers to table $1 B ;{ }^{2}$ core-plug measurements unless otherwise noted; ${ }^{3}$ see reference list.]

\begin{tabular}{|c|c|c|c|}
\hline $\begin{array}{l}\text { Data } \\
\text { ID }\end{array}$ & Identification $^{2}$ (source $^{3}$ ) & Age & Location \\
\hline 1 & $\begin{array}{l}\text { El Paso Natural Gas well, } \\
\text { 1-Wagon Wheel (authors' } \\
\text { data) }\end{array}$ & $\begin{array}{l}\text { Tertiary and } \\
\text { Cretaceous }\end{array}$ & $\begin{array}{l}\text { Green River basin, } \\
\text { Wyoming, Sec.5, } \\
\text { T.30N.,R.108W. }\end{array}$ \\
\hline 2 & $\begin{array}{l}\text { Dakota Sandstone, Bridger Lake } \\
\text { field (Ben E. Law, 1987, } \\
\text { written commun.) }\end{array}$ & Cretaceous & $\begin{array}{l}\text { North flank of Uinta } \\
\text { Mountains, Utah }\end{array}$ \\
\hline 3 & $\begin{array}{l}\text { J sandstone (Schmoker and } \\
\text { Higley, 1991) }\end{array}$ & Cretaceous & $\begin{array}{l}\text { Denver basin, } \\
\text { Colorado }\end{array}$ \\
\hline 4 & $\begin{array}{l}\text { COST GE-1 well (Ama to and } \\
\text { Bebout, 1978; Scholle, 1979) }\end{array}$ & Cretaceous & $\begin{array}{l}\text { Georgia embayment, } \\
\text { offshore eastern } \\
\text { Florida }\end{array}$ \\
\hline 5 & CosT B-2 well (Scholle, 1977) & Cretaceous & $\begin{array}{l}\text { Baltimore Canyon } \\
\text { Trough, of fshore } \\
\text { New Jersey }\end{array}$ \\
\hline 6 & $\begin{array}{l}\text { Unspecified sandstones from } \\
\text { several wells (method } \\
\text { unknown, interpreted and } \\
\text { modified by Bostick, 1979) }\end{array}$ & $\begin{array}{l}\text { Cenozoic and } \\
\text { Cretaceous }\end{array}$ & $\begin{array}{l}\text { Northern Sakhal in } \\
\text { Island, USSR }\end{array}$ \\
\hline $7 a$ & Nugget Sands tone & $\begin{array}{c}\text { Jurassic and } \\
\text { Triassic }\end{array}$ & $\begin{array}{l}\text { Overthrust belt, } \\
\text { northern Utah }\end{array}$ \\
\hline b & Tuscarora Sandstone & Silurian & $\begin{array}{l}\text { Valley and Ridge } \\
\text { Province, } \\
\text { Pennsylvania }\end{array}$ \\
\hline$c$ & $\begin{array}{l}\text { Bromide Formation } \\
\text { ( } 7 \mathrm{a}, \mathrm{b} \text {, and c are all } \\
\text { point-count data, } \\
\text { Houseknecht, 1988) }\end{array}$ & Ordovician & $\begin{array}{l}\text { Central oklahoma } \\
\text { Platform }\end{array}$ \\
\hline 8 & $\begin{array}{l}\text { Canning Formation (Kenneth } \mathrm{J} \text {. } \\
\text { Bird, 1987, written } \\
\text { commun.) }\end{array}$ & $\begin{array}{l}\text { Cretaceous } \\
\text { and early } \\
\text { Tertiary }\end{array}$ & North Slope, Alaska \\
\hline
\end{tabular}

9 Thomson sand (Kenneth J. Bird, Cretaceous North Slope, Alaska 1987, written commun.) 
Table 1A. (continued)

\begin{tabular}{|c|c|c|c|}
\hline $\begin{array}{l}\text { Data } \\
\underline{\text { ID }}\end{array}$ & Identification $^{2}$ (source $\left.^{3}\right)$ & Age & Location \\
\hline 10 & $\begin{array}{l}\text { Mobile-Texaco Citnalta I-59 } \\
\text { well (sonic-log data, } \\
\text { Schmidt and McDonald, 1979; } \\
\text { Issler, 1984) }\end{array}$ & $\begin{array}{l}\text { Cretaceous } \\
\text { and } \\
\text { Jurassic }\end{array}$ & $\begin{array}{l}\text { Scotian Shelf, } \\
\text { offshore eastern } \\
\text { Canada }\end{array}$ \\
\hline 11 & $\begin{array}{l}\text { Almond Formation (Ben E. Law, } \\
\text { 1988, written commun.) }\end{array}$ & Cretaceous & $\begin{array}{l}\text { Green River basin, } \\
\text { Wyoming }\end{array}$ \\
\hline 12 & COST 1 well (Turner, 1983a) & $\begin{array}{l}\text { 01igocene and } \\
\text { Eocene }\end{array}$ & Norton Sound, Alaska \\
\hline 13 & COST 2 well (Turner, 1983b) & $\begin{array}{l}\text { 0ligocene and } \\
\text { Eocene }\end{array}$ & Norton Sound, Alaska \\
\hline 14 & CoST 1 well (Turner, 1984a) & Miocene & $\begin{array}{l}\text { Navarin basin, Bering } \\
\text { Sea, Alaska }\end{array}$ \\
\hline 15 & COST 1 well (Turner, 1984b) & $\begin{array}{l}\text { Pliocene and } \\
\text { Eocene }\end{array}$ & $\begin{array}{l}\text { St. George basin, } \\
\text { Bering Sea, Alaska }\end{array}$ \\
\hline 16 & COST 2 well (Turner, 1984c) & $\begin{array}{l}\text { Pliocene and } \\
\text { Eocene; } \\
\text { Jurassic }\end{array}$ & $\begin{array}{l}\text { St. George basin, } \\
\text { Bering Sea, Alaska }\end{array}$ \\
\hline 17 & $\begin{array}{l}\text { COST } 1 \text { well (Wills and others, } \\
\text { 1978; Magoon, 1986) }\end{array}$ & $\begin{array}{l}\text { Cretaceous- } \\
\text { Jurassic }\end{array}$ & $\begin{array}{l}\text { Lower Cook Inlet, } \\
\text { Alaska }\end{array}$ \\
\hline 18 & $\begin{array}{l}\text { Kodiak Shelf Stratigraphic } \\
\text { Test Wells } 1 \text { and } 2 \text { (Turner, } \\
\text { 1987) }\end{array}$ & Eocene & $\begin{array}{l}\text { Kodiak Shelf, western } \\
\text { Gulf of Alaska }\end{array}$ \\
\hline 19 & COST 1 well (Turner, 1988) & $\begin{array}{l}\text { Miocene, } \\
\text { 0ligocene, } \\
\text { and Eocene }\end{array}$ & $\begin{array}{c}\text { North Aleutian Shelf, } \\
\text { Bering Sea, Alaska }\end{array}$ \\
\hline 20 & $\begin{array}{l}\text { Hibernia Formation (point- } \\
\text { count data, Brown and } \\
\text { others, 1989) }\end{array}$ & $\begin{array}{l}\text { Cretaceous } \\
\text { and } \\
\text { Jurassic }\end{array}$ & $\begin{array}{l}\text { Jeanne d'Arc basin, } \\
\text { Grand Banks of } \\
\text { Newfoundland }\end{array}$ \\
\hline 21 & $\begin{array}{l}\text { Norphlet Formation (point- } \\
\text { count data, McBride and } \\
\text { others, 1987) }\end{array}$ & Jurassic & $\begin{array}{l}\text { Gulf Coast basin, } \\
\text { Mississippi and } \\
\text { Alabama }\end{array}$ \\
\hline 22 & $\begin{array}{l}\text { Norphlet Formation (Honda, } \\
\text { 1981) }\end{array}$ & Jurassic & $\begin{array}{l}\text { Gulf Coast basin, } \\
\text { Alabama }\end{array}$ \\
\hline
\end{tabular}


Table 1A. (continued)

\begin{tabular}{|c|c|c|c|}
\hline $\begin{array}{l}\text { Data }_{1} \\
\text { ID }\end{array}$ & Identification $^{2}$ (source $\left.^{3}\right)$ & Age & Location \\
\hline 23 & $\begin{array}{l}\text { Paleogene Arkoses (highest } \\
\text { and average values only, } \\
\text { Helmold and Van de Kamp, } \\
\text { 1984) }\end{array}$ & $\begin{array}{l}\text { 0ligocene, } \\
\text { Eocene, and } \\
\text { Paleocene }\end{array}$ & $\begin{array}{l}\text { Santa Ynez Mountains, } \\
\text { California }\end{array}$ \\
\hline 24 & $\begin{array}{l}\text { Cerro Prieto geothermal field } \\
\text { (density-log data, Barker, } \\
\text { 1979, Lyons and Van de Kamp, } \\
\text { 1980) }\end{array}$ & $\begin{array}{l}\text { Pleis tocene } \\
\text { and } \\
\text { Pliocene }\end{array}$ & $\begin{array}{l}\text { Colorado River delta, } \\
\text { Baja California, } \\
\text { Mexico }\end{array}$ \\
\hline 25 & $\begin{array}{l}\text { Norphlet Formation (highest } \\
\text { values only, Dixon and } \\
\text { others, 1989) }\end{array}$ & Jurassic & $\begin{array}{l}\text { Gulf Coast basin, } \\
\text { southwest Alabama } \\
\text { and vicinity }\end{array}$ \\
\hline 26 & $\begin{array}{l}\text { Amoco M.G. 1-Eischeid well } \\
\text { (authors' log data) } \\
\text { Co., Iowa, Sec.6, } \\
\text { T.83N.,R.35W. }\end{array}$ & $\begin{array}{l}\text { Cambrian and } \\
\text { Precambrian }\end{array}$ & $\begin{array}{l}\text { Midcontinent Rift } \\
\text { System, Carroll }\end{array}$ \\
\hline 27 & $\begin{array}{l}\text { Upper Minnelusa Formation } \\
\text { (authors' log data) }\end{array}$ & Permian & $\begin{array}{l}\text { West Mellott field, } \\
\text { Powder River basin, } \\
\text { Wyoming, T.52N., } \\
\text { R.68W. }\end{array}$ \\
\hline 28 & $\begin{array}{l}\text { Fangst Group of Heidrum field } \\
\text { (Harris, 1989) }\end{array}$ & Jurassic & $\begin{array}{l}\text { Hal tenbanken area, } \\
\text { offshore, } \\
\text { mid-Norway }\end{array}$ \\
\hline 29 & $\begin{array}{l}\text { Well H-22, Venture field } \\
\text { (mixed log and core data, } \\
\text { Mudford and Best, 1989) }\end{array}$ & $\begin{array}{l}\text { Cretaceous } \\
\text { and } \\
\text { Jurassic }\end{array}$ & $\begin{array}{l}\text { Scotian Shelf (Sable } \\
\text { Island), offshore } \\
\text { eastern Canada }\end{array}$ \\
\hline 30 & $\begin{array}{l}\text { Upper Minnelusa, B sand } \\
\text { (James, 1989) }\end{array}$ & Permian & $\begin{array}{l}\text { Hawk Point field, } \\
\text { Powder River basin, } \\
\text { Wyoming }\end{array}$ \\
\hline 31 & $\begin{array}{l}\text { Upper Minnelusa (point-count } \\
\text { data, Schenk, 1990, written } \\
\text { commun.) }\end{array}$ & Permian & $\begin{array}{l}\text { Deep Reno area, } \\
\text { Powder River basin, } \\
\text { Wyoming }\end{array}$ \\
\hline 32 & $\begin{array}{l}\text { Tuscher and Farrer Formations, } \\
\text { Exxon, 1-Wilkin Ridge well }\end{array}$ & Cretaceous & $\begin{array}{l}\text { Central Uinta basin, } \\
\text { Utah }\end{array}$ \\
\hline
\end{tabular}


Table 1A. (continued)

\section{$\underline{\text { ID }}_{1}^{\text {Identification }^{2} \text { (source }^{3} \text { ) }}$ \\ 33a Green River Formation \\ b Weber Formation \\ c Wasatch Formation \\ d Castlegate Sandstone \\ e Mesaverde Group \\ (33a, b, c, d, and e, are \\ all authors' data; Anders, 1990)}

Age

Location

34 Miocene sandstones (mixed log and core data, Taylor, 1990)

35 Louden Properties, Good, Lahr, and Kaufman well (Streib, 1981; Laughrey and Harper, 1986)

36a Castlegate Sandstone

b Blackhawk Formation (36a and $b$ are from Exxon Production Research Company 2-Sego Canyon well;

Wendlandt and Bhuyan, 1990; R.C. Johnson, 1990, written commun.)

37a Wasatch Formation

b Tuscher Formation

c Neslen Formation

d Blackhawk Formation

(37a, b, c, and d are all from Colorado Interstate Gas Exploration, 21-Natural Buttes well; J.K. Pittman, 1990, written commun.)
Eocene

Pennsylvanian and Permian

Eocene

Cretaceous

Cretaceous

Miocene

\author{
Miocene
}

Devonian

Devonian

Cretaceous

Cretaceous

Tertiary and Cretaceous

Tertiary and Cretaceous

Tertiary and Cretaceous

Tertiary and Cretaceous
Uinta basin, Utah

Uinta basin, Utah

Uinta basin, Utah

Uinta basin, Utah

Uinta basin, Utah

\section{Corsair trend, Brazos area, offshore Texas Gulf Coast \\ Appalachian basin, Pennsylvania}

Book Cliffs area, Grand County, Utah Book Cliffs area, Grand County, Utah

\author{
Uinta basin, Utah \\ Uinta basin, Utah \\ Uinta basin, Utah \\ Uinta basin, Utah
}


Table 1A. (continued)

\section{$\underline{\text { ID }}^{\text {ata }_{1}} \quad$ Identification $^{2}$ (source $^{3}$ ) \\ 38 Green River Formation of Pariette Bench field (Pitman and others, 1982) \\ 39 Mesaverde Group, Pacific Gas Trans. Co., 1-7 Federal- Natural well (authors' data) \\ 40 Green River-Wasatch Formations, Havenstride $0 i l$ Co., 72X-22B well (authors' data)}

41 Cozzette sandstone member of Iles Formation, marine lower part of Mesaverde Group,

Celeron 0 il and Gas Co., 35-1 Porter Mountain Federal well (authors' data;

Johnson and Nuccio, 1986)

42 Mancos "B", CER Corp., PTS 22-12 Federal well (authors' data; Johnson and Nuccio, 1986)

43 Non-marine upper part of Mesaverde Group, Twin Arrow, 4-14 $\mathrm{C}$ and $\mathrm{K}$ well (authors' data; Johnson and Nuccio, 1986)

44 Non-marine upper part of Mesaverde Group, CER Geonuclear, RBE-01 and RB-MHF-3 wells (authors' data; Hansley and Johnson, 1980; Johnson and Nuccio, 1986)
Age

Location

Eocene

Uinta basin, Utah

Cretaceous

$$
\begin{aligned}
& \text { Uinta basin, Uintah } \\
& \text { Co., Utah, Sec.7, } \\
& \text { T.11S.,R.21E. }
\end{aligned}
$$

Eocene

Cretaceous

Piceance Creek basin, Mesa Co., Colorado, Sec. 35, T.9S., R.92W.

Cretaceous

Cretaceous

Cretaceous

Piceance Creek basin, Rio Blanco Co., Colorado, Sec.14, T.3S.,R.98W., and Sec.11,T.3S., R.98W. , respectively 
Table 1A. (continued)
${ }_{\text {ID }}^{\text {Data }} 1$ Identification ${ }^{2}$ (source $^{3}$ )
45a Cozzette sandstone
b Cocoran sands tone
c Rollins sands tone
(45a, b, and c are all
marine lower part of
Mesaverde Group, CER Corp.,
MWX wells 1,2 , and 3;
authors' data; Bostick
and Freeman, 1984)
46 Non-marine upper part of Mesaverde Group, CER Corp. MWX wells 1,2 , and 3
(authors' data; Bostick and Freeman, 1984)
47 Ellesmerian sequence (sonic- log porosity, Van de Kamp, 1988)
48 Sussex Sandstone Member of Cody Shale (Debra K. Higley, 1991, written commun.)

Age

Location

Cretaceous

Cretaceous

Cretaceous

Cretaceous

Jurassic, Triassic, and

Permian

Cretaceous
Piceance Creek basin Piceance Creek basin Piceance Creek basin (all wells are in Garfield Co., Colorado, Sec.34, T.6S.,R.94W.

Piceance Creek basin, Garfield Co., Colorado, Sec.34, T.6S., R. 94W.

North Slope of Alaska

Powder River basin, Wyoming 
Table 1B. BOX-DIAGRAM POROSITY PBRCENTILES

$\left[^{1}\right.$ Data-identification number from table $1 \mathrm{~A} ;{ }^{2}$ single highest porosity measurement; ${ }^{3} \mathrm{ft} \times 1000 ;{ }^{4}$ equivalent vitrinite reflectance $(\%) ;{ }^{5}$ number of porosity measurements.]

\begin{tabular}{|c|c|c|c|c|c|c|c|c|c|}
\hline $\begin{array}{l}\text { Data } \\
\underline{\text { ID }}^{1}\end{array}$ & 10 th & $\begin{array}{l}\text { Poros: } \\
25 \text { th } \\
\end{array}$ & $\begin{array}{l}\text { yer } \\
50 \text { th } \\
\end{array}$ & $\begin{array}{l}\text { atiles } \\
75 \text { th }\end{array}$ & 90th & High $^{2}$ & Depth $^{3}$ & $\underline{\mathrm{R}}_{\theta}^{4}$ & $\begin{array}{c}\text { Data } \\
\text { Points }\end{array}$ \\
\hline 1 & 7.5 & 8.7 & 11.5 & 16.5 & 18.2 & 20.4 & 5.0 & 0.23 & 48 \\
\hline 1 & 4.4 & 5.4 & 7.2 & 10.8 & 13.4 & 13.8 & 7.1 & 0.52 & 17 \\
\hline 1 & 6.7 & 9.1 & 11.2 & 12.4 & 13.4 & 16.0 & 7.3 & 0.56 & 44 \\
\hline 1 & 5.1 & 8.2 & 11.0 & 12.5 & 13.7 & 14.9 & 8.1 & 0.66 & 75 \\
\hline 1 & 4.3 & 5.3 & 6.9 & 9.0 & 10.6 & 13.6 & 8.9 & 0.78 & 76 \\
\hline 1 & 5.7 & 7.3 & 8.7 & 10.2 & 11.2 & 13.0 & 10.2 & 0.96 & 93 \\
\hline 1 & 3.2 & 3.7 & 4.8 & 6.8 & 8.4 & 10.8 & 11.0 & 1.07 & 72 \\
\hline 1 & 4.3 & 5.2 & 6.3 & 7.9 & 10.5 & 12.8 & 13.1 & 1.37 & 84 \\
\hline 1 & 1.4 & 2.1 & 3.2 & 4.4 & 5.1 & 5.4 & 14.9 & 1.62 & 9 \\
\hline 1 & 3.0 & 3.6 & 4.2 & 4.7 & 5.0 & 6.4 & 16.1 & 1.78 & 33 \\
\hline 2 & 4.5 & 6.6 & 9.8 & 13.2 & 15.3 & 20.5 & 15.5 & 0.59 & 326 \\
\hline 3 & 10.5 & 12.0 & 14.5 & 16.5 & 18.0 & 20.1 & 5.8 & 0.59 & 26 \\
\hline 3 & 10.0 & 12.5 & 18.0 & 20.5 & 22.0 & 25.0 & 5.0 & 0.41 & 32 \\
\hline 3 & 14.0 & 16.0 & 20.5 & 21.0 & 21.5 & 22.1 & 5.1 & 0.45 & 9 \\
\hline 3 & 12.5 & 16.0 & 19.5 & 21.0 & 23.0 & 23.5 & 4.8 & 0.50 & 8 \\
\hline 3 & 10.0 & 12.5 & 17.0 & 19.0 & 21.0 & 22.9 & 5.9 & 0.52 & 60 \\
\hline 3 & 17.0 & 18.0 & 19.5 & 21.0 & 22.5 & 22.6 & 5.3 & 0.53 & 18 \\
\hline 3 & 8.0 & 10.0 & 15.5 & 19.5 & 20.0 & 20.3 & 5.8 & 0.57 & 9 \\
\hline 3 & 6.5 & 9.5 & 13.5 & 17.5 & 18.0 & 21.7 & 6.2 & 0.57 & 18 \\
\hline 3 & 7.0 & 10.5 & 13.5 & 19.5 & 21.0 & 24.4 & 6.4 & 0.58 & 18 \\
\hline 3 & 11.5 & 15.0 & 16.5 & 18.0 & 19.5 & 23.6 & 5.1 & 0.61 & 24 \\
\hline 3 & 13.5 & 14.0 & 15.0 & 16.0 & 18.0 & 19.0 & 5.6 & 0.61 & 10 \\
\hline 3 & 18.0 & 20.0 & 23.0 & 26.0 & 26.5 & 28.0 & 4.3 & 0.62 & 25 \\
\hline 3 & 9.0 & 13.5 & 16.5 & 18.5 & 19.5 & 21.2 & 6.5 & 0.62 & 21 \\
\hline 3 & 11.0 & 12.0 & 13.5 & 14.5 & 15.0 & 15.8 & 6.8 & 0.62 & 15 \\
\hline 3 & 13.5 & 16.0 & 18.0 & 20.5 & 23.0 & 24.9 & 5.6 & 0.63 & 32 \\
\hline 3 & 14.5 & 16.0 & 18.0 & 20.0 & 21.5 & 24.8 & 6.3 & 0.63 & 97 \\
\hline 3 & 8.0 & 8.5 & 11.0 & 12.5 & 16.0 & 18.6 & 7.5 & 0.64 & 19 \\
\hline 3 & 8.0 & 9.5 & 10.5 & 12.0 & 13.5 & 16.4 & 7.9 & 0.65 & 47 \\
\hline 3 & 5.5 & 10.5 & 14.0 & 16.0 & 17.5 & 19.7 & 6.2 & 0.67 & 41 \\
\hline 3 & 9.5 & 11.0 & 12.0 & 13.5 & 14.0 & 15.3 & 6.5 & 0.80 & 45 \\
\hline 3 & 6.0 & 8.0 & 9.5 & 11.0 & 11.5 & 12.0 & 7.4 & 0.80 & 50 \\
\hline 3 & 5.0 & 6.5 & 8.5 & 9.0 & 11.5 & 11.5 & 7.4 & 0.81 & 21 \\
\hline 3 & 10.5 & 11.0 & 12.0 & 13.0 & 14.0 & 15.8 & 7.3 & 0.82 & 45 \\
\hline 3 & 5.5 & 7.5 & 10.0 & 11.0 & 11.5 & 11.7 & 7.8 & 0.82 & 12 \\
\hline
\end{tabular}


Table 1B. (continued)

\begin{tabular}{|c|c|c|c|c|c|c|c|c|c|}
\hline $\begin{array}{r}\text { Data } \\
\text { ID1 }\end{array}$ & $10 \mathrm{th}$ & $\begin{array}{c}\text { Porosi } \\
25 \mathrm{th}\end{array}$ & $\begin{array}{l}\text { Perc } \\
50 \text { th } \\
\end{array}$ & $\begin{array}{l}\text { tiles } \\
75 \text { th }\end{array}$ & 90 th & $\mathrm{High}^{2}$ & Depth $^{3}$ & $\underline{R}_{0}^{4}$ & $\begin{array}{c}\text { Data } \\
\text { Points }\end{array}$ \\
\hline $\begin{array}{l}3 \\
3 \\
3 \\
3 \\
3\end{array}$ & $\begin{array}{l}5.5 \\
7.5 \\
6.0 \\
5.0 \\
4.5\end{array}$ & $\begin{array}{r}7.0 \\
10.0 \\
7.0 \\
7.0 \\
6.0\end{array}$ & $\begin{array}{r}9.5 \\
12.5 \\
7.5 \\
8.5 \\
7.0\end{array}$ & $\begin{array}{r}12.5 \\
15.0 \\
8.5 \\
9.5 \\
10.5\end{array}$ & $\begin{array}{r}14.5 \\
18.5 \\
9.0 \\
10.0 \\
14.5\end{array}$ & $\begin{array}{r}18.6 \\
20.8 \\
9.1 \\
11.1 \\
15.3\end{array}$ & $\begin{array}{l}8.3 \\
6.8 \\
8.7 \\
7.5 \\
7.8\end{array}$ & $\begin{array}{l}0.82 \\
0.68 \\
0.90 \\
1.07 \\
1.10\end{array}$ & $\begin{array}{l}45 \\
37 \\
10 \\
28 \\
30\end{array}$ \\
\hline $\begin{array}{l}3 \\
3 \\
4 \\
4 \\
4\end{array}$ & $\begin{array}{l}2.2 \\
2.6 \\
6.2 \\
4.7 \\
4.2\end{array}$ & $\begin{array}{r}3.5 \\
5.0 \\
11.5 \\
6.2 \\
6.1\end{array}$ & $\begin{array}{r}6.0 \\
7.5 \\
18.5 \\
12.0 \\
9.5\end{array}$ & $\begin{array}{r}8.0 \\
9.5 \\
23.3 \\
18.2 \\
13.7\end{array}$ & $\begin{array}{r}9.5 \\
11.0 \\
25.6 \\
19.9 \\
15.8\end{array}$ & $\begin{array}{l}10.6 \\
15.5 \\
29.5 \\
21.0 \\
18.5\end{array}$ & $\begin{array}{r}7.6 \\
8.0 \\
8.4 \\
9.5 \\
10.6\end{array}$ & $\begin{array}{l}1.14 \\
1.14 \\
0.43 \\
0.62 \\
0.75\end{array}$ & $\begin{array}{l}57 \\
54 \\
44 \\
34 \\
33\end{array}$ \\
\hline $\begin{array}{l}5 \\
5 \\
5 \\
5 \\
5\end{array}$ & $\begin{array}{r}22.6 \\
22.1 \\
11.2 \\
6.1 \\
8.4\end{array}$ & $\begin{array}{r}28.2 \\
30.8 \\
16.5 \\
8.7 \\
12.5\end{array}$ & $\begin{array}{l}32.8 \\
33.2 \\
18.9 \\
13.0 \\
19.0\end{array}$ & $\begin{array}{l}35.8 \\
34.2 \\
22.8 \\
17.3 \\
23.9\end{array}$ & $\begin{array}{l}39.7 \\
37.9 \\
26.6 \\
23.8 \\
27.6\end{array}$ & $\begin{array}{l}41.0 \\
38.0 \\
29.3 \\
28.0 \\
32.5\end{array}$ & $\begin{array}{l}5.5 \\
6.5 \\
8.5 \\
9.2 \\
9.7\end{array}$ & $\begin{array}{l}0.40 \\
0.43 \\
0.49 \\
0.52 \\
0.54\end{array}$ & $\begin{array}{l}13 \\
11 \\
42 \\
32 \\
38\end{array}$ \\
\hline $\begin{array}{l}5 \\
5 \\
5 \\
5 \\
5\end{array}$ & $\begin{array}{r}16.3 \\
6.6 \\
4.8 \\
4.4 \\
5.4\end{array}$ & $\begin{array}{r}16.7 \\
11.3 \\
8.4 \\
7.2 \\
6.6\end{array}$ & $\begin{array}{r}18.0 \\
12.5 \\
10.3 \\
11.1 \\
8.2\end{array}$ & $\begin{array}{r}20.0 \\
14.0 \\
12.0 \\
13.0 \\
9.6\end{array}$ & $\begin{array}{l}20.6 \\
19.5 \\
19.4 \\
14.7 \\
10.6\end{array}$ & $\begin{array}{l}21.0 \\
19.5 \\
20.0 \\
16.4 \\
11.0\end{array}$ & $\begin{array}{l}10.5 \\
11.5 \\
12.6 \\
13.6 \\
14.7\end{array}$ & $\begin{array}{l}0.56 \\
0.60 \\
0.66 \\
0.73 \\
0.80\end{array}$ & $\begin{array}{r}8 \\
16 \\
16 \\
40 \\
17\end{array}$ \\
\hline $\begin{array}{l}6 \\
6 \\
6 \\
6 \\
6\end{array}$ & $\begin{array}{r}17.9 \\
17.3 \\
11.2 \\
6.2 \\
7.1\end{array}$ & $\begin{array}{r}20.2 \\
19.8 \\
13.5 \\
8.2 \\
8.4\end{array}$ & $\begin{array}{l}22.5 \\
23.5 \\
18.0 \\
12.0 \\
11.5\end{array}$ & $\begin{array}{l}23.8 \\
26.8 \\
23.5 \\
20.2 \\
20.5\end{array}$ & $\begin{array}{l}29.4 \\
27.8 \\
24.8 \\
21.9 \\
23.7\end{array}$ & $\begin{array}{l}33.0 \\
34.0 \\
28.0 \\
29.0 \\
27.0\end{array}$ & $\begin{array}{l}--- \\
--- \\
--- \\
--- \\
---\end{array}$ & $\begin{array}{l}0.30 \\
0.35 \\
0.39 \\
0.44 \\
0.50\end{array}$ & $\begin{array}{r}9 \\
13 \\
26 \\
22 \\
23\end{array}$ \\
\hline $\begin{array}{l}6 \\
6 \\
6 \\
7 a \\
b\end{array}$ & $\begin{array}{l}5.6 \\
4.6 \\
3.0 \\
1.6 \\
0.6\end{array}$ & $\begin{array}{l}7.8 \\
6.2 \\
4.8 \\
3.3 \\
1.7\end{array}$ & $\begin{array}{r}11.0 \\
8.8 \\
6.5 \\
5.6 \\
3.6\end{array}$ & $\begin{array}{r}17.0 \\
12.8 \\
8.5 \\
8.2 \\
6.0\end{array}$ & $\begin{array}{r}22.2 \\
13.8 \\
11.8 \\
10.3 \\
8.5\end{array}$ & $\begin{array}{l}25.0 \\
15.0 \\
13.0 \\
13.0 \\
18.4\end{array}$ & $\begin{array}{r}- \\
- \\
-11.7 \\
11.0\end{array}$ & $\begin{array}{l}0.55 \\
0.61 \\
0.66 \\
1.04 \\
1.55\end{array}$ & $\begin{array}{r}26 \\
13 \\
11 \\
69 \\
111\end{array}$ \\
\hline $\begin{array}{l}8^{c} \\
8 \\
8 \\
9\end{array}$ & $\begin{array}{r}0.2 \\
13.6 \\
10.8 \\
10.8 \\
16.4\end{array}$ & $\begin{array}{r}0.5 \\
14.5 \\
13.2 \\
12.2 \\
19.5\end{array}$ & $\begin{array}{r}0.9 \\
17.0 \\
16.0 \\
18.1 \\
23.5\end{array}$ & $\begin{array}{r}1.7 \\
19.5 \\
18.5 \\
19.8 \\
25.0\end{array}$ & $\begin{array}{r}2.6 \\
21.4 \\
20.4 \\
22.2 \\
26.2\end{array}$ & $\begin{array}{r}5.5 \\
21.6 \\
23.1 \\
22.8 \\
26.6\end{array}$ & $\begin{array}{r}0.1 \\
11.6 \\
12.6 \\
11.7 \\
13.8\end{array}$ & $\begin{array}{l}4.50 \\
0.57 \\
0.60 \\
0.66 \\
0.61\end{array}$ & $\begin{array}{r}88 \\
12 \\
26 \\
17 \\
8\end{array}$ \\
\hline
\end{tabular}


Table 1B. (continued)

\begin{tabular}{|c|c|c|c|c|c|c|c|c|c|}
\hline $\begin{array}{r}\text { Data } \\
\text { ID1 }\end{array}$ & 10 th & $\begin{array}{c}\text { Porosi } \\
25 \text { th } \\
\end{array}$ & $\begin{array}{r}\text { Perc } \\
50 \text { th } \\
\end{array}$ & $\begin{array}{l}\text { tiles } \\
75 \text { th } \\
\end{array}$ & 90 th & $\underline{\mathrm{High}}^{2}$ & $\underline{\text { Depth }}^{3}$ & $\underline{R}_{0}^{4}$ & $\begin{array}{c}\text { Data } \\
\text { Points }\end{array}$ \\
\hline $\begin{array}{r}9 \\
10 \\
10 \\
10 \\
10\end{array}$ & $\begin{array}{l}12.0 \\
24.8 \\
22.6 \\
17.7 \\
10.5\end{array}$ & $\begin{array}{l}13.5 \\
29.6 \\
24.3 \\
20.0 \\
14.6\end{array}$ & $\begin{array}{l}14.7 \\
31.7 \\
26.0 \\
21.7 \\
15.1\end{array}$ & $\begin{array}{l}15.8 \\
33.3 \\
33.8 \\
22.5 \\
16.0\end{array}$ & $\begin{array}{l}19.0 \\
34.4 \\
34.2 \\
25.3 \\
18.5\end{array}$ & $\begin{array}{l}21.8 \\
35.5 \\
34.5 \\
26.0 \\
19.0\end{array}$ & $\begin{array}{r}12.9 \\
5.5 \\
7.1 \\
8.3 \\
9.8\end{array}$ & $\begin{array}{l}0.72 \\
0.34 \\
0.40 \\
0.46 \\
0.53\end{array}$ & $\begin{array}{l}10 \\
13 \\
11 \\
12 \\
10\end{array}$ \\
\hline $\begin{array}{l}10 \\
10 \\
10 \\
11 \\
11\end{array}$ & $\begin{array}{r}11.6 \\
6.5 \\
5.3 \\
3.4 \\
4.7\end{array}$ & $\begin{array}{r}13.3 \\
7.4 \\
6.5 \\
4.1 \\
8.2\end{array}$ & $\begin{array}{r}15.1 \\
9.7 \\
7.5 \\
4.9 \\
10.2\end{array}$ & $\begin{array}{r}16.8 \\
14.7 \\
8.2 \\
5.9 \\
11.4\end{array}$ & $\begin{array}{r}20.4 \\
20.4 \\
10.7 \\
7.4 \\
12.0\end{array}$ & $\begin{array}{r}23.5 \\
21.0 \\
11.5 \\
7.9 \\
12.4\end{array}$ & $\begin{array}{r}11.0 \\
12.4 \\
13.8 \\
9.9 \\
13.1\end{array}$ & $\begin{array}{l}0.60 \\
0.69 \\
0.79 \\
0.82 \\
1.30\end{array}$ & $\begin{array}{r}11 \\
11 \\
8 \\
13 \\
44\end{array}$ \\
\hline $\begin{array}{l}11 \\
11 \\
11 \\
11 \\
11\end{array}$ & $\begin{array}{r}8.2 \\
12.4 \\
3.1 \\
2.5 \\
11.1\end{array}$ & $\begin{array}{r}9.2 \\
14.2 \\
3.9 \\
4.4 \\
12.8\end{array}$ & $\begin{array}{r}10.3 \\
17.2 \\
4.7 \\
5.6 \\
14.1\end{array}$ & $\begin{array}{r}12.1 \\
18.2 \\
6.3 \\
6.5 \\
15.2\end{array}$ & $\begin{array}{r}13.3 \\
18.7 \\
7.7 \\
7.0 \\
16.0\end{array}$ & $\begin{array}{r}14.0 \\
18.9 \\
10.0 \\
7.6 \\
17.1\end{array}$ & $\begin{array}{r}12.4 \\
7.5 \\
9.1 \\
11.9 \\
7.0\end{array}$ & $\begin{array}{l}1.20 \\
0.60 \\
0.80 \\
1.60 \\
0.59\end{array}$ & $\begin{array}{l}39 \\
13 \\
23 \\
30 \\
21\end{array}$ \\
\hline $\begin{array}{l}11 \\
11 \\
11 \\
11 \\
11\end{array}$ & $\begin{array}{r}12.6 \\
2.7 \\
7.8 \\
8.0 \\
2.2\end{array}$ & $\begin{array}{r}14.1 \\
3.9 \\
10.4 \\
10.6 \\
3.1\end{array}$ & $\begin{array}{r}14.7 \\
7.0 \\
12.3 \\
13.4 \\
3.9\end{array}$ & $\begin{array}{r}15.7 \\
11.9 \\
13.4 \\
15.0 \\
4.9\end{array}$ & $\begin{array}{r}16.7 \\
14.8 \\
15.8 \\
17.5 \\
5.8\end{array}$ & $\begin{array}{r}17.5 \\
17.7 \\
16.1 \\
19.2 \\
6.2\end{array}$ & $\begin{array}{l}6.8 \\
6.6 \\
8.4 \\
6.9 \\
8.6\end{array}$ & $\begin{array}{l}0.71 \\
0.73 \\
0.70 \\
0.75 \\
0.84\end{array}$ & $\begin{array}{l}32 \\
92 \\
25 \\
40 \\
17\end{array}$ \\
\hline $\begin{array}{l}11 \\
11 \\
11 \\
11 \\
11\end{array}$ & $\begin{array}{l}5.3 \\
1.5 \\
5.3 \\
3.2 \\
1.6\end{array}$ & $\begin{array}{l}6.1 \\
3.1 \\
6.2 \\
4.2 \\
2.2\end{array}$ & $\begin{array}{l}7.7 \\
3.6 \\
6.6 \\
6.2 \\
3.0\end{array}$ & $\begin{array}{l}9.6 \\
4.2 \\
7.1 \\
9.1 \\
4.8\end{array}$ & $\begin{array}{r}10.6 \\
5.0 \\
7.7 \\
12.0 \\
7.4\end{array}$ & $\begin{array}{r}11.0 \\
5.7 \\
9.1 \\
16.3 \\
8.1\end{array}$ & $\begin{array}{r}11.0 \\
9.1 \\
12.5 \\
10.6 \\
14.3\end{array}$ & $\begin{array}{l}0.76 \\
0.80 \\
1.64 \\
1.12 \\
1.34\end{array}$ & $\begin{array}{l}21 \\
20 \\
63 \\
91 \\
29\end{array}$ \\
\hline $\begin{array}{l}11 \\
11 \\
11 \\
11 \\
11\end{array}$ & $\begin{array}{r}4.5 \\
15.6 \\
20.1 \\
17.3 \\
7.0\end{array}$ & $\begin{array}{r}6.8 \\
16.9 \\
20.9 \\
18.2 \\
9.3\end{array}$ & $\begin{array}{r}8.5 \\
20.2 \\
22.2 \\
20.2 \\
11.4\end{array}$ & $\begin{array}{r}9.6 \\
21.7 \\
23.5 \\
21.5 \\
12.5\end{array}$ & $\begin{array}{l}10.5 \\
22.6 \\
24.0 \\
23.4 \\
13.1\end{array}$ & $\begin{array}{l}11.5 \\
23.2 \\
25.6 \\
23.9 \\
15.2\end{array}$ & $\begin{array}{r}13.7 \\
4.5 \\
4.5 \\
4.8 \\
9.0\end{array}$ & $\begin{array}{l}1.64 \\
0.67 \\
0.57 \\
0.68 \\
0.65\end{array}$ & $\begin{array}{l}15 \\
26 \\
23 \\
18 \\
90\end{array}$ \\
\hline $\begin{array}{l}11 \\
11 \\
12 \\
13 \\
13\end{array}$ & $\begin{array}{r}14.3 \\
10.3 \\
7.8 \\
14.9 \\
8.4\end{array}$ & $\begin{array}{r}14.8 \\
12.4 \\
8.9 \\
17.2 \\
11.2\end{array}$ & $\begin{array}{r}17.5 \\
13.5 \\
9.8 \\
20.8 \\
13.0\end{array}$ & $\begin{array}{l}20.6 \\
14.9 \\
10.8 \\
21.9 \\
15.2\end{array}$ & $\begin{array}{l}21.4 \\
15.9 \\
11.5 \\
28.1 \\
16.6\end{array}$ & $\begin{array}{l}21.4 \\
16.7 \\
12.3 \\
28.5 \\
17.5\end{array}$ & $\begin{array}{r}5.8 \\
4.6 \\
10.9 \\
7.0 \\
8.3\end{array}$ & $\begin{array}{l}0.62 \\
0.60 \\
0.69 \\
0.41 \\
0.47\end{array}$ & $\begin{array}{r}13 \\
13 \\
68 \\
9 \\
14\end{array}$ \\
\hline
\end{tabular}


Table 1B. (continued)

\begin{tabular}{|c|c|c|c|c|c|c|c|c|c|}
\hline $\begin{array}{r}\text { Data } \\
\text { ID1 }\end{array}$ & 10 th & $\begin{array}{r}\text { Poros } \\
25 \text { th } \\
\end{array}$ & $\begin{array}{r}\text { Perc } \\
50 \text { th } \\
\end{array}$ & $\begin{array}{l}\text { itiles } \\
75 \text { th }\end{array}$ & 90 th & $\mathrm{High}^{2}$ & Depth $^{3}$ & $\underline{R}_{\theta}^{4}$ & $\begin{array}{c}\text { Data } \\
\text { Points }\end{array}$ \\
\hline $\begin{array}{l}13 \\
14 \\
15 \\
15 \\
15\end{array}$ & $\begin{array}{r}7.6 \\
28.9 \\
30.8 \\
35.5 \\
24.7\end{array}$ & $\begin{array}{r}9.6 \\
30.1 \\
32.2 \\
36.4 \\
26.2\end{array}$ & $\begin{array}{l}11.0 \\
32.5 \\
33.6 \\
37.1 \\
28.4\end{array}$ & $\begin{array}{l}11.8 \\
35.4 \\
42.2 \\
38.2 \\
29.9\end{array}$ & $\begin{array}{l}12.5 \\
37.1 \\
43.9 \\
40.2 \\
31.1\end{array}$ & $\begin{array}{l}12.8 \\
37.3 \\
47.1 \\
40.8 \\
36.6\end{array}$ & $\begin{array}{r}13.4 \\
4.3 \\
3.4 \\
4.1 \\
5.7\end{array}$ & $\begin{array}{l}1.25 \\
0.33 \\
0.46 \\
0.50 \\
0.58\end{array}$ & $\begin{array}{r}31 \\
9 \\
48 \\
15 \\
101\end{array}$ \\
\hline $\begin{array}{l}15 \\
15 \\
15 \\
15 \\
16\end{array}$ & $\begin{array}{r}5.3 \\
16.2 \\
14.6 \\
4.1 \\
34.8\end{array}$ & $\begin{array}{r}12.7 \\
17.5 \\
15.6 \\
8.9 \\
36.8\end{array}$ & $\begin{array}{l}27.2 \\
20.5 \\
17.6 \\
13.2 \\
37.7\end{array}$ & $\begin{array}{l}30.8 \\
22.3 \\
19.0 \\
15.5 \\
38.8\end{array}$ & $\begin{array}{l}31.1 \\
23.4 \\
20.2 \\
20.9 \\
39.5\end{array}$ & $\begin{array}{l}33.8 \\
24.5 \\
22.7 \\
25.0 \\
41.3\end{array}$ & $\begin{array}{r}6.8 \\
7.9 \\
9.7 \\
10.3 \\
4.1\end{array}$ & $\begin{array}{l}0.64 \\
0.72 \\
0.85 \\
0.92 \\
0.27\end{array}$ & $\begin{array}{l}22 \\
53 \\
50 \\
11 \\
26\end{array}$ \\
\hline $\begin{array}{l}16 \\
16 \\
16 \\
16 \\
16\end{array}$ & $\begin{array}{r}24.9 \\
18.3 \\
14.0 \\
7.6 \\
13.5\end{array}$ & $\begin{array}{r}27.6 \\
18.7 \\
21.6 \\
9.6 \\
15.6\end{array}$ & $\begin{array}{l}28.8 \\
22.9 \\
22.4 \\
17.2 \\
18.6\end{array}$ & $\begin{array}{l}29.9 \\
24.0 \\
22.7 \\
18.4 \\
20.6\end{array}$ & $\begin{array}{l}30.5 \\
25.9 \\
23.8 \\
18.9 \\
21.4\end{array}$ & $\begin{array}{l}30.6 \\
26.2 \\
24.6 \\
23.3 \\
22.0\end{array}$ & $\begin{array}{r}5.2 \\
7.6 \\
8.5 \\
9.5 \\
10.1\end{array}$ & $\begin{array}{l}0.30 \\
0.37 \\
0.40 \\
0.43 \\
0.47\end{array}$ & $\begin{array}{r}9 \\
12 \\
14 \\
13 \\
40\end{array}$ \\
\hline $\begin{array}{l}16 \\
16 \\
16 \\
16 \\
16\end{array}$ & $\begin{array}{r}11.3 \\
12.6 \\
6.6 \\
4.5 \\
1.9\end{array}$ & $\begin{array}{r}12.4 \\
13.1 \\
10.5 \\
5.0 \\
2.6\end{array}$ & $\begin{array}{r}13.2 \\
13.6 \\
12.2 \\
6.7 \\
3.4\end{array}$ & $\begin{array}{r}13.6 \\
13.8 \\
12.9 \\
8.7 \\
3.9\end{array}$ & $\begin{array}{r}14.8 \\
14.1 \\
13.5 \\
9.4 \\
5.0\end{array}$ & $\begin{array}{r}15.9 \\
14.5 \\
14.3 \\
10.2 \\
5.0\end{array}$ & $\begin{array}{l}10.6 \\
11.8 \\
12.5 \\
14.2 \\
14.6\end{array}$ & $\begin{array}{l}0.50 \\
0.57 \\
0.61 \\
0.74 \\
0.79\end{array}$ & $\begin{array}{r}32 \\
27 \\
36 \\
18 \\
9\end{array}$ \\
\hline $\begin{array}{l}17 \\
17 \\
17 \\
17 \\
18\end{array}$ & $\begin{array}{l}8.6 \\
2.4 \\
0.6 \\
2.0 \\
2.0\end{array}$ & $\begin{array}{r}10.4 \\
2.8 \\
1.4 \\
2.3 \\
3.7\end{array}$ & $\begin{array}{r}12.8 \\
3.2 \\
2.2 \\
2.6 \\
5.4\end{array}$ & $\begin{array}{r}17.7 \\
3.5 \\
2.9 \\
3.3 \\
5.9\end{array}$ & $\begin{array}{r}19.4 \\
4.5 \\
3.2 \\
3.9 \\
6.2\end{array}$ & $\begin{array}{r}19.5 \\
6.7 \\
3.4 \\
4.4 \\
6.6\end{array}$ & $\begin{array}{r}5.4 \\
7.1 \\
9.3 \\
12.4 \\
8.1\end{array}$ & $\begin{array}{l}0.39 \\
0.45 \\
0.55 \\
0.69 \\
0.58\end{array}$ & $\begin{array}{l}15 \\
27 \\
17 \\
31 \\
32\end{array}$ \\
\hline $\begin{array}{l}18 \\
19 \\
19 \\
19 \\
19\end{array}$ & $\begin{array}{l}17.4 \\
34.3 \\
26.5 \\
24.4 \\
26.8\end{array}$ & $\begin{array}{l}19.2 \\
34.4 \\
28.5 \\
25.8 \\
29.3\end{array}$ & $\begin{array}{l}20.3 \\
34.9 \\
30.0 \\
27.7 \\
30.7\end{array}$ & $\begin{array}{l}23.2 \\
35.4 \\
31.4 \\
31.6 \\
31.2\end{array}$ & $\begin{array}{l}25.4 \\
36.2 \\
33.2 \\
32.8 \\
31.6\end{array}$ & $\begin{array}{l}25.9 \\
36.7 \\
33.6 \\
33.4 \\
31.8\end{array}$ & $\begin{array}{r}10.3 \\
5.2 \\
6.0 \\
8.1 \\
8.6\end{array}$ & $\begin{array}{l}0.54 \\
0.32 \\
0.34 \\
0.38 \\
0.40\end{array}$ & $\begin{array}{r}9 \\
9 \\
17 \\
20 \\
16\end{array}$ \\
\hline $\begin{array}{l}19 \\
19 \\
19 \\
19 \\
20\end{array}$ & $\begin{array}{r}10.0 \\
6.4 \\
2.0 \\
7.3 \\
4.9\end{array}$ & $\begin{array}{r}17.2 \\
13.8 \\
6.9 \\
7.4 \\
10.4\end{array}$ & $\begin{array}{r}17.8 \\
18.2 \\
9.3 \\
8.3 \\
14.8\end{array}$ & $\begin{array}{l}19.3 \\
19.7 \\
10.7 \\
12.4 \\
17.2\end{array}$ & $\begin{array}{l}21.0 \\
21.8 \\
11.2 \\
13.1 \\
20.3\end{array}$ & $\begin{array}{l}29.0 \\
24.1 \\
12.5 \\
13.6 \\
22.0\end{array}$ & $\begin{array}{l}10.0 \\
11.1 \\
15.4 \\
16.7 \\
12.5\end{array}$ & $\begin{array}{l}0.45 \\
0.50 \\
0.82 \\
1.02 \\
0.55\end{array}$ & $\begin{array}{r}35 \\
10 \\
12 \\
8 \\
37\end{array}$ \\
\hline
\end{tabular}


Table 1B. (continued)

\begin{tabular}{|c|c|c|c|c|c|c|c|c|c|}
\hline $\begin{array}{r}\text { Da ta } \\
\text { ID1 }\end{array}$ & 10 th & $\begin{array}{l}\text { Porosi } \\
25 \text { th }\end{array}$ & $\begin{array}{r}\text { Perc } \\
50 \text { th } \\
\end{array}$ & $\begin{array}{l}\text { tiles } \\
75 \text { th }\end{array}$ & 90 th & $\mathrm{High}^{2}$ & Depth $^{3}$ & $\underline{R}_{\theta}^{4}$ & $\begin{array}{c}\text { Data } \\
\text { Points } \\
\end{array}$ \\
\hline $\begin{array}{l}21 \\
21 \\
21 \\
21 \\
22\end{array}$ & $\begin{array}{l}1.0 \\
7.1 \\
0.7 \\
1.3 \\
7.2\end{array}$ & $\begin{array}{l}2.2 \\
9.8 \\
0.9 \\
3.5 \\
8.2\end{array}$ & $\begin{array}{r}6.8 \\
10.8 \\
1.3 \\
10.5 \\
9.8\end{array}$ & $\begin{array}{r}9.4 \\
11.5 \\
3.5 \\
13.0 \\
11.8\end{array}$ & $\begin{array}{l}15.0 \\
14.9 \\
18.7 \\
19.7 \\
14.4\end{array}$ & $\begin{array}{l}16.0 \\
15.0 \\
19.0 \\
20.0 \\
16.9\end{array}$ & $\begin{array}{l}16.2 \\
19.9 \\
21.4 \\
22.4 \\
18.6\end{array}$ & $\begin{array}{l}1.56 \\
2.02 \\
2.25 \\
2.40 \\
1.66\end{array}$ & $\begin{array}{r}26 \\
12 \\
8 \\
8 \\
27\end{array}$ \\
\hline $\begin{array}{l}22 \\
22 \\
23 \\
23 \\
23\end{array}$ & $\begin{array}{r}10.2 \\
7.0 \\
- \\
- \\
-\end{array}$ & $\begin{array}{l}11.3 \\
10.5 \\
---- \\
---- \\
----\end{array}$ & $\begin{array}{l}13.1 \\
12.4 \\
22.1 \\
16.1 \\
14.4\end{array}$ & $\begin{array}{l}13.9 \\
13.9 \\
---- \\
---- \\
----\end{array}$ & $\begin{array}{l}15.0 \\
15.1 \\
--- \\
---\end{array}$ & $\begin{array}{l}15.4 \\
18.0 \\
34.0 \\
26.4 \\
21.3\end{array}$ & $\begin{array}{l}18.5 \\
18.5 \\
-\ldots- \\
-\cdots \\
-\end{array}$ & $\begin{array}{l}1.64 \\
1.64 \\
0.29 \\
0.42 \\
0.65\end{array}$ & $\begin{array}{r}32 \\
240 \\
--- \\
--- \\
---\end{array}$ \\
\hline $\begin{array}{l}23 \\
23 \\
23 \\
23 \\
23\end{array}$ & $\begin{array}{l}---- \\
---- \\
---- \\
---- \\
----\end{array}$ & $\begin{array}{l}---- \\
---- \\
---- \\
---- \\
----\end{array}$ & $\begin{array}{r}13.7 \\
7.7 \\
8.3 \\
3.8 \\
2.6\end{array}$ & $\begin{array}{l}---- \\
---- \\
---- \\
---- \\
----\end{array}$ & $\begin{array}{l}---- \\
---- \\
---- \\
---- \\
----\end{array}$ & $\begin{array}{r}32.9 \\
9.3 \\
12.3 \\
7.2 \\
6.7\end{array}$ & $\begin{array}{l}--- \\
--- \\
--- \\
--- \\
----\end{array}$ & $\begin{array}{l}0.35 \\
0.59 \\
0.73 \\
1.80 \\
2.58\end{array}$ & $\begin{array}{l}--- \\
--- \\
--- \\
--- \\
---\end{array}$ \\
\hline $\begin{array}{l}23 \\
23 \\
23 \\
23 \\
23\end{array}$ & $\begin{array}{l}--- \\
--- \\
---- \\
--- \\
----\end{array}$ & $\begin{array}{l}---- \\
---- \\
---- \\
---- \\
----\end{array}$ & $\begin{array}{r}9.4 \\
11.0 \\
3.9 \\
1.5 \\
2.5\end{array}$ & $\begin{array}{l}---- \\
---- \\
---- \\
---- \\
----\end{array}$ & $\begin{array}{l}---- \\
---- \\
---- \\
---- \\
----\end{array}$ & $\begin{array}{r}10.7 \\
12.3 \\
5.5 \\
1.7 \\
3.1\end{array}$ & $\begin{array}{l}--- \\
--- \\
--- \\
--- \\
----\end{array}$ & $\begin{array}{l}0.86 \\
0.30 \\
0.80 \\
1.50 \\
2.50\end{array}$ & $\begin{array}{l}--- \\
--- \\
--- \\
--- \\
---\end{array}$ \\
\hline $\begin{array}{l}24 \\
24 \\
24 \\
24 \\
24\end{array}$ & $\begin{array}{r}12.6 \\
6.1 \\
18.9 \\
26.1 \\
21.1\end{array}$ & $\begin{array}{r}13.8 \\
7.2 \\
26.2 \\
27.8 \\
23.8\end{array}$ & $\begin{array}{l}15.7 \\
10.7 \\
29.5 \\
28.8 \\
25.0\end{array}$ & $\begin{array}{l}18.2 \\
12.0 \\
31.4 \\
29.3 \\
26.8\end{array}$ & $\begin{array}{l}19.2 \\
13.4 \\
38.1 \\
30.2 \\
27.4\end{array}$ & $\begin{array}{l}24.0 \\
14.5 \\
39.0 \\
30.5 \\
28.0\end{array}$ & $\begin{array}{l}6.4 \\
8.3 \\
5.4 \\
3.6 \\
4.5\end{array}$ & $\begin{array}{l}2.00 \\
2.35 \\
2.85 \\
0.45 \\
0.56\end{array}$ & $\begin{array}{l}34 \\
24 \\
34 \\
16 \\
12\end{array}$ \\
\hline $\begin{array}{l}24 \\
24 \\
24 \\
25 \\
25\end{array}$ & $\begin{array}{l}33.9 \\
33.7 \\
32.6 \\
- \\
-\end{array}$ & $\begin{array}{l}34.4 \\
34.4 \\
33.5 \\
- \\
-\end{array}$ & $\begin{array}{l}36.0 \\
35.9 \\
36.0 \\
--- \\
---\end{array}$ & $\begin{array}{l}37.4 \\
36.9 \\
37.2 \\
- \\
-\end{array}$ & $\begin{array}{l}38.2 \\
37.6 \\
38.3 \\
- \\
- \\
---\end{array}$ & $\begin{array}{l}40.0 \\
38.0 \\
39.0 \\
27.6 \\
22.9\end{array}$ & $\begin{array}{r}0.9 \\
2.0 \\
3.0 \\
12.5 \\
13.6\end{array}$ & $\begin{array}{l}0.31 \\
0.40 \\
0.50 \\
0.74 \\
0.81\end{array}$ & $\begin{array}{r}18 \\
18 \\
12 \\
--- \\
---\end{array}$ \\
\hline $\begin{array}{l}25 \\
25 \\
25 \\
25 \\
25\end{array}$ & $\begin{array}{l}--- \\
---- \\
--- \\
--- \\
----\end{array}$ & $\begin{array}{l}---- \\
---- \\
---- \\
---- \\
----\end{array}$ & $\begin{array}{l}---- \\
---- \\
---- \\
---- \\
----\end{array}$ & $\begin{array}{l}---- \\
---- \\
---- \\
---- \\
----\end{array}$ & $\begin{array}{l}--- \\
---- \\
---- \\
---- \\
----\end{array}$ & $\begin{array}{l}23.7 \\
19.5 \\
16.6 \\
24.0 \\
20.0\end{array}$ & $\begin{array}{l}14.9 \\
15.3 \\
15.5 \\
15.6 \\
15.7\end{array}$ & $\begin{array}{l}0.94 \\
1.16 \\
1.18 \\
1.19 \\
1.20\end{array}$ & $\begin{array}{l}--- \\
--- \\
--- \\
--- \\
---\end{array}$ \\
\hline
\end{tabular}


Table 1B. (continued)

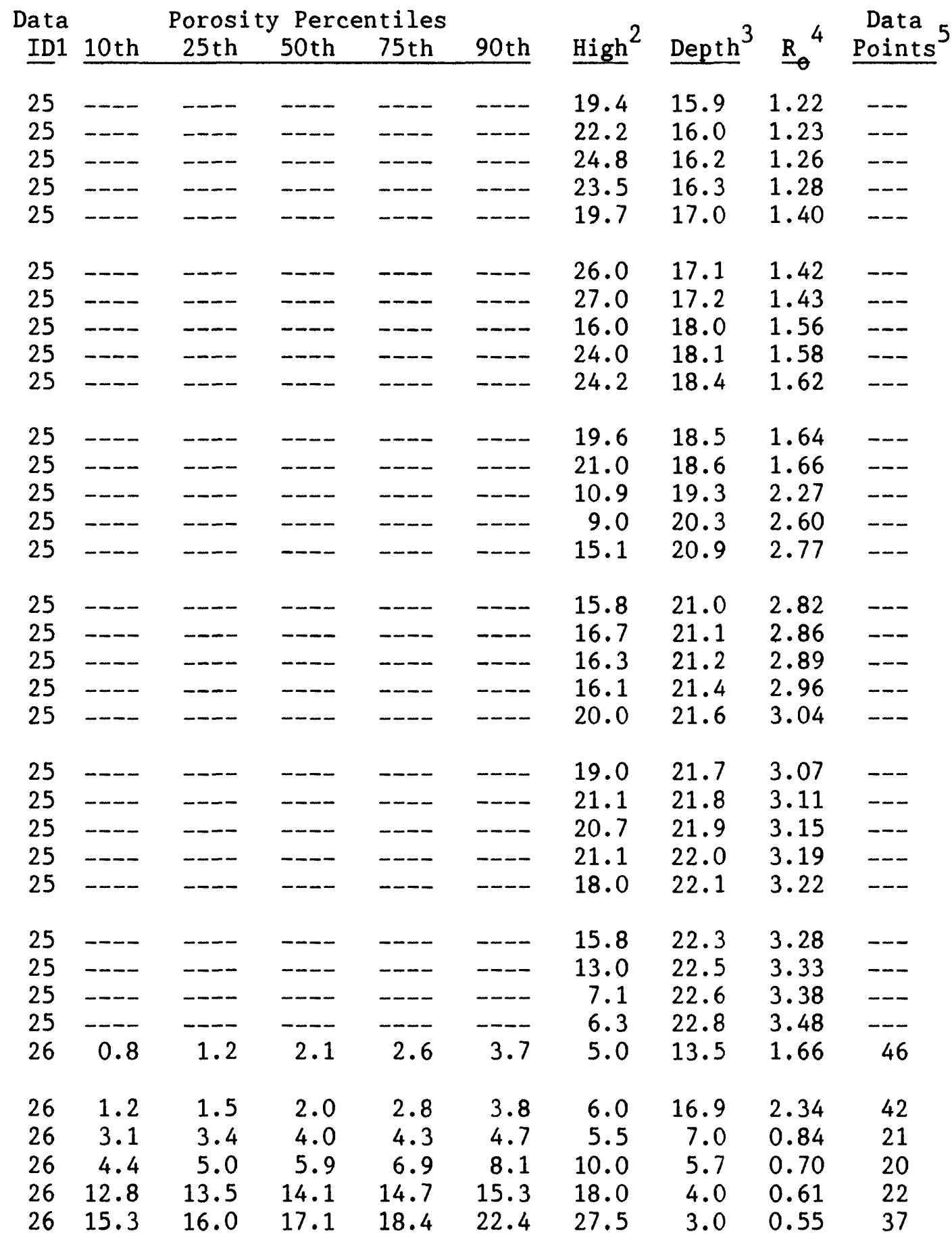


Table 1B. (continued)

\begin{tabular}{|c|c|c|c|c|c|c|c|c|c|}
\hline $\begin{array}{r}\text { Data } \\
\text { ID1 }\end{array}$ & $10 \mathrm{th}$ & $\begin{array}{c}\text { Porosi } \\
25 \mathrm{th}\end{array}$ & $\begin{array}{l}\text { Perc } \\
50 \text { th }\end{array}$ & $\begin{array}{l}\text { tiles } \\
75 \mathrm{th}\end{array}$ & 90 th & $\mathrm{High}^{2}$ & Depth $^{3}$ & $\mathrm{R}^{4}$ & $\begin{array}{c}\text { Data } \\
\text { Points }\end{array}$ \\
\hline $\begin{array}{l}27 \\
28 \\
29 \\
29 \\
29\end{array}$ & $\begin{array}{r}7.0 \\
26.8 \\
24.4 \\
22.8 \\
18.5\end{array}$ & $\begin{array}{l}10.0 \\
30.1 \\
25.0 \\
24.5 \\
21.5\end{array}$ & $\begin{array}{l}12.0 \\
32.6 \\
29.5 \\
27.5 \\
23.0\end{array}$ & $\begin{array}{l}17.5 \\
34.5 \\
30.0 \\
28.5 \\
24.2\end{array}$ & $\begin{array}{l}21.5 \\
35.9 \\
30.6 \\
30.2 \\
24.7\end{array}$ & $\begin{array}{l}--- \\
39.0 \\
31.0 \\
31.0 \\
25.0\end{array}$ & $\begin{array}{l}7.2 \\
8.1 \\
6.3 \\
7.8 \\
8.8\end{array}$ & $\begin{array}{l}0.59 \\
0.40 \\
0.40 \\
0.45 \\
0.50\end{array}$ & $\begin{array}{r}20 \\
87 \\
8 \\
8 \\
10\end{array}$ \\
\hline $\begin{array}{l}29 \\
29 \\
29 \\
29 \\
29\end{array}$ & $\begin{array}{r}14.2 \\
6.1 \\
9.3 \\
10.5 \\
13.3\end{array}$ & $\begin{array}{r}16.2 \\
9.7 \\
9.8 \\
13.2 \\
15.9\end{array}$ & $\begin{array}{l}17.5 \\
12.5 \\
11.2 \\
16.1 \\
19.5\end{array}$ & $\begin{array}{l}18.8 \\
18.5 \\
11.9 \\
18.2 \\
22.6\end{array}$ & $\begin{array}{l}21.5 \\
22.9 \\
13.1 \\
20.5 \\
23.8\end{array}$ & $\begin{array}{l}22.0 \\
23.5 \\
14.0 \\
21.0 \\
25.0\end{array}$ & $\begin{array}{l}10.7 \\
12.8 \\
14.4 \\
15.5 \\
16.1\end{array}$ & $\begin{array}{l}0.58 \\
0.71 \\
0.80 \\
0.90 \\
0.95\end{array}$ & $\begin{array}{r}14 \\
11 \\
9 \\
25 \\
27\end{array}$ \\
\hline $\begin{array}{l}29 \\
29 \\
29 \\
30 \\
31\end{array}$ & $\begin{array}{r}12.9 \\
9.0 \\
10.0 \\
4.3 \\
0.4\end{array}$ & $\begin{array}{r}16.1 \\
9.9 \\
10.8 \\
6.2 \\
1.0\end{array}$ & $\begin{array}{r}18.2 \\
11.5 \\
11.7 \\
9.0 \\
2.9\end{array}$ & $\begin{array}{r}21.2 \\
14.6 \\
13.2 \\
10.4 \\
9.8\end{array}$ & $\begin{array}{l}24.6 \\
15.9 \\
14.0 \\
11.5 \\
14.6\end{array}$ & $\begin{array}{l}26.0 \\
16.0 \\
14.5 \\
18.0 \\
23.0\end{array}$ & $\begin{array}{l}16.4 \\
17.1 \\
17.6 \\
11.4 \\
15.2\end{array}$ & $\begin{array}{l}0.99 \\
1.04 \\
1.10 \\
0.92 \\
1.50\end{array}$ & $\begin{array}{r}38 \\
11 \\
10 \\
8 \\
37\end{array}$ \\
\hline $\begin{array}{l}32 \\
32 \\
32 \\
33 a \\
a\end{array}$ & $\begin{array}{l}2.8 \\
4.6 \\
1.2 \\
1.1 \\
5.3\end{array}$ & $\begin{array}{l}5.1 \\
5.7 \\
1.5 \\
2.1 \\
7.0\end{array}$ & $\begin{array}{l}5.6 \\
6.5 \\
2.0 \\
3.0 \\
9.6\end{array}$ & $\begin{array}{r}6.2 \\
7.0 \\
2.8 \\
4.5 \\
11.2\end{array}$ & $\begin{array}{r}6.7 \\
7.7 \\
4.5 \\
6.6 \\
12.8\end{array}$ & $\begin{array}{r}6.9 \\
8.0 \\
4.9 \\
7.8 \\
18.5\end{array}$ & $\begin{array}{r}10.3 \\
10.8 \\
11.2 \\
8.2 \\
5.6\end{array}$ & $\begin{array}{l}0.74 \\
0.84 \\
0.94 \\
0.68 \\
0.49\end{array}$ & $\begin{array}{r}15 \\
55 \\
10 \\
24 \\
138\end{array}$ \\
\hline $\begin{array}{l}a \\
a \\
a \\
a \\
b\end{array}$ & $\begin{array}{l}5.9 \\
1.2 \\
2.3 \\
3.9 \\
2.0\end{array}$ & $\begin{array}{l}8.0 \\
2.2 \\
2.9 \\
6.0 \\
2.4\end{array}$ & $\begin{array}{l}8.7 \\
2.8 \\
3.7 \\
9.3 \\
2.8\end{array}$ & $\begin{array}{r}10.0 \\
3.7 \\
6.0 \\
13.9 \\
3.2\end{array}$ & $\begin{array}{r}10.8 \\
4.8 \\
9.6 \\
16.5 \\
3.5\end{array}$ & $\begin{array}{r}11.3 \\
5.2 \\
10.8 \\
22.2 \\
4.2\end{array}$ & $\begin{array}{r}5.3 \\
5.3 \\
5.3 \\
5.4 \\
14.6\end{array}$ & $\begin{array}{l}0.48 \\
0.48 \\
0.48 \\
0.45 \\
1.70\end{array}$ & $\begin{array}{r}28 \\
12 \\
24 \\
213 \\
33\end{array}$ \\
\hline $\begin{array}{l}b \\
c \\
d \\
e \\
e\end{array}$ & $\begin{array}{l}9.6 \\
2.1 \\
7.8 \\
1.5 \\
2.8\end{array}$ & $\begin{array}{r}11.0 \\
2.8 \\
8.4 \\
2.0 \\
5.0\end{array}$ & $\begin{array}{r}12.6 \\
4.8 \\
9.4 \\
2.4 \\
6.6\end{array}$ & $\begin{array}{r}13.4 \\
9.5 \\
11.2 \\
4.8 \\
7.7\end{array}$ & $\begin{array}{r}13.9 \\
11.4 \\
11.8 \\
7.6 \\
8.4\end{array}$ & $\begin{array}{r}15.2 \\
17.9 \\
12.0 \\
9.1 \\
9.6\end{array}$ & $\begin{array}{r}3.3 \\
5.5 \\
8.2 \\
19.3 \\
8.3\end{array}$ & $\begin{array}{l}0.48 \\
0.58 \\
0.91 \\
2.40 \\
0.83\end{array}$ & $\begin{array}{r}16 \\
121 \\
35 \\
13 \\
45\end{array}$ \\
\hline $\begin{array}{l}34 \\
34 \\
34 \\
34 \\
34\end{array}$ & $\begin{array}{r}16.4 \\
12.4 \\
12.4 \\
7.0 \\
8.3\end{array}$ & $\begin{array}{r}22.8 \\
15.8 \\
13.9 \\
9.2 \\
11.5\end{array}$ & $\begin{array}{l}24.2 \\
19.0 \\
17.0 \\
12.8 \\
12.8\end{array}$ & $\begin{array}{l}27.2 \\
20.1 \\
19.1 \\
17.9 \\
14.5\end{array}$ & $\begin{array}{l}30.0 \\
21.6 \\
22.6 \\
20.7 \\
16.9\end{array}$ & $\begin{array}{l}30.5 \\
22.5 \\
25.5 \\
21.5 \\
18.5\end{array}$ & $\begin{array}{r}7.8 \\
10.5 \\
12.4 \\
13.3 \\
14.2\end{array}$ & $\begin{array}{l}0.40 \\
0.58 \\
0.78 \\
0.92 \\
1.10\end{array}$ & $\begin{array}{r}9 \\
9 \\
19 \\
25 \\
16\end{array}$ \\
\hline
\end{tabular}


Table 1B. (continued)

\begin{tabular}{|c|c|c|c|c|c|c|c|c|c|}
\hline $\begin{array}{r}\text { Da ta } \\
\text { ID1 }\end{array}$ & $10 \mathrm{th}$ & $\begin{array}{r}\text { Poros } \\
25 \text { th } \\
\end{array}$ & $\begin{array}{r}\text { Perc } \\
50 \text { th } \\
\end{array}$ & $\begin{array}{c}\text { tiles } \\
75 \text { th }\end{array}$ & 90 th & $\mathrm{High}^{2}$ & Depth $^{3}$ & $\underline{R}_{\theta}^{4}$ & $\begin{array}{c}\text { Data } \\
\text { Points } \\
\end{array}$ \\
\hline $\begin{array}{l}34 \\
34 \\
34 \\
34 \\
34\end{array}$ & $\begin{array}{r}5.9 \\
4.8 \\
8.6 \\
9.0 \\
13.2\end{array}$ & $\begin{array}{r}11.5 \\
8.8 \\
10.6 \\
11.2 \\
16.2\end{array}$ & $\begin{array}{l}14.2 \\
12.0 \\
12.9 \\
15.7 \\
21.2\end{array}$ & $\begin{array}{l}16.8 \\
14.4 \\
14.2 \\
19.8 \\
25.9\end{array}$ & $\begin{array}{l}18.3 \\
15.4 \\
15.1 \\
21.6 \\
27.4\end{array}$ & $\begin{array}{l}20.5 \\
16.5 \\
17.5 \\
24.5 \\
28.5\end{array}$ & $\begin{array}{l}14.8 \\
15.6 \\
16.0 \\
16.4 \\
17.0\end{array}$ & $\begin{array}{l}1.22 \\
1.40 \\
1.54 \\
1.65 \\
1.85\end{array}$ & $\begin{array}{l}24 \\
13 \\
21 \\
39 \\
33\end{array}$ \\
\hline $\begin{array}{r}35 \\
36 a \\
b \\
37 a \\
b\end{array}$ & $\begin{array}{r}1.5 \\
9.4 \\
14.3 \\
2.4 \\
1.8\end{array}$ & $\begin{array}{r}4.2 \\
15.1 \\
18.1 \\
3.0 \\
5.5\end{array}$ & $\begin{array}{r}6.5 \\
18.2 \\
19.9 \\
3.8 \\
6.5\end{array}$ & $\begin{array}{r}8.7 \\
20.6 \\
21.3 \\
4.7 \\
8.0\end{array}$ & $\begin{array}{r}9.9 \\
21.6 \\
22.2 \\
5.4 \\
10.2\end{array}$ & $\begin{array}{r}10.0 \\
22.4 \\
23.3 \\
5.4 \\
10.8\end{array}$ & $\begin{array}{l}2.8 \\
0.7 \\
0.7 \\
4.5 \\
6.4\end{array}$ & $\begin{array}{l}1.85 \\
0.56 \\
0.57 \\
0.56 \\
0.75\end{array}$ & $\begin{array}{r}35 \\
33 \\
33 \\
11 \\
8\end{array}$ \\
\hline $\begin{array}{l}\quad \begin{array}{l}c \\
d \\
38 \\
38 \\
39\end{array}\end{array}$ & $\begin{array}{l}2.7 \\
3.2 \\
5.2 \\
6.8 \\
5.4\end{array}$ & $\begin{array}{l}3.8 \\
4.2 \\
6.6 \\
8.8 \\
6.5\end{array}$ & $\begin{array}{r}5.8 \\
7.0 \\
10.2 \\
9.9 \\
7.5\end{array}$ & $\begin{array}{r}6.8 \\
9.2 \\
12.9 \\
14.2 \\
8.2\end{array}$ & $\begin{array}{r}8.6 \\
10.3 \\
14.7 \\
16.2 \\
9.6\end{array}$ & $\begin{array}{r}9.0 \\
10.3 \\
15.8 \\
16.2 \\
10.3\end{array}$ & $\begin{array}{l}7.5 \\
8.5 \\
4.9 \\
4.9 \\
7.0\end{array}$ & $\begin{array}{l}0.83 \\
0.95 \\
0.47 \\
0.47 \\
0.79\end{array}$ & $\begin{array}{l}14 \\
14 \\
37 \\
15 \\
24\end{array}$ \\
\hline $\begin{array}{l}39 \\
40 \\
41 \\
41 \\
41\end{array}$ & $\begin{array}{l}4.4 \\
7.2 \\
8.2 \\
5.2 \\
3.0\end{array}$ & $\begin{array}{l}5.6 \\
8.8 \\
8.4 \\
5.8 \\
3.5\end{array}$ & $\begin{array}{r}8.2 \\
11.5 \\
8.6 \\
6.8 \\
4.2\end{array}$ & $\begin{array}{r}8.7 \\
14.6 \\
9.1 \\
7.5 \\
5.0\end{array}$ & $\begin{array}{r}9.1 \\
16.2 \\
9.8 \\
8.5 \\
5.3\end{array}$ & $\begin{array}{r}9.2 \\
16.6 \\
9.9 \\
9.3 \\
5.4\end{array}$ & $\begin{array}{l}7.7 \\
6.2 \\
8.2 \\
8.2 \\
8.2\end{array}$ & $\begin{array}{l}0.87 \\
0.62 \\
1.33 \\
1.33 \\
1.33\end{array}$ & $\begin{array}{r}19 \\
15 \\
9 \\
20 \\
24\end{array}$ \\
\hline $\begin{array}{l}42 \\
42 \\
42 \\
42 \\
43\end{array}$ & $\begin{array}{r}4.6 \\
3.7 \\
4.6 \\
4.7 \\
13.0\end{array}$ & $\begin{array}{r}4.9 \\
3.9 \\
4.9 \\
4.8 \\
16.6\end{array}$ & $\begin{array}{r}5.1 \\
4.7 \\
5.1 \\
5.0 \\
19.4\end{array}$ & $\begin{array}{r}5.5 \\
5.5 \\
5.4 \\
5.3 \\
21.2\end{array}$ & $\begin{array}{r}5.7 \\
5.6 \\
5.6 \\
5.5 \\
22.5\end{array}$ & $\begin{array}{r}5.9 \\
5.6 \\
5.7 \\
7.3 \\
24.6\end{array}$ & $\begin{array}{r}11.7 \\
11.7 \\
11.8 \\
11.8 \\
1.1\end{array}$ & $\begin{array}{l}1.80 \\
1.80 \\
1.80 \\
1.80 \\
0.54\end{array}$ & $\begin{array}{l}26 \\
12 \\
12 \\
17 \\
50\end{array}$ \\
\hline $\begin{array}{l}44 \\
44 \\
45 a \\
b \\
c\end{array}$ & $\begin{array}{l}4.2 \\
5.0 \\
3.5 \\
2.2 \\
3.8\end{array}$ & $\begin{array}{l}5.4 \\
6.1 \\
5.6 \\
3.9 \\
5.1\end{array}$ & $\begin{array}{l}6.9 \\
8.1 \\
6.2 \\
4.6 \\
5.9\end{array}$ & $\begin{array}{l}8.0 \\
9.0 \\
6.9 \\
5.3 \\
7.4\end{array}$ & $\begin{array}{r}9.5 \\
11.1 \\
7.6 \\
5.9 \\
7.9\end{array}$ & $\begin{array}{r}10.9 \\
12.4 \\
8.9 \\
6.0 \\
8.0\end{array}$ & $\begin{array}{l}6.2 \\
6.0 \\
7.9 \\
8.1 \\
7.5\end{array}$ & $\begin{array}{l}0.97 \\
0.93 \\
2.08 \\
2.16 \\
1.89\end{array}$ & $\begin{array}{l}25 \\
29 \\
81 \\
15 \\
13\end{array}$ \\
\hline $\begin{array}{l}46 \\
46 \\
46 \\
46 \\
46\end{array}$ & $\begin{array}{l}2.8 \\
5.6 \\
4.2 \\
4.3 \\
4.0\end{array}$ & $\begin{array}{l}4.6 \\
6.1 \\
4.6 \\
6.0 \\
5.1\end{array}$ & $\begin{array}{l}5.7 \\
6.9 \\
5.0 \\
6.9 \\
6.4\end{array}$ & $\begin{array}{l}6.9 \\
7.4 \\
5.6 \\
7.6 \\
7.9\end{array}$ & $\begin{array}{l}7.7 \\
8.6 \\
6.0 \\
8.3 \\
9.1\end{array}$ & $\begin{array}{r}9.5 \\
9.7 \\
8.1 \\
9.2 \\
11.1\end{array}$ & $\begin{array}{l}4.3 \\
4.6 \\
4.7 \\
4.8 \\
4.9\end{array}$ & $\begin{array}{l}0.88 \\
0.93 \\
0.96 \\
1.00 \\
1.02\end{array}$ & $\begin{array}{l}51 \\
26 \\
23 \\
28 \\
74\end{array}$ \\
\hline
\end{tabular}


Table 1B. (continued)

\begin{tabular}{|c|c|c|c|c|c|c|c|c|c|}
\hline $\begin{array}{r}\text { Data } \\
\text { ID1 }\end{array}$ & $10 \mathrm{th}$ & $\begin{array}{r}\text { Poros } \\
25 \text { th } \\
\end{array}$ & $\begin{array}{r}\text { Perc } \\
50 \text { th } \\
\end{array}$ & $\begin{array}{l}\text { tiles } \\
75 \mathrm{th} \\
\end{array}$ & 90 th & $\mathrm{High}^{2}$ & Depth $^{3}$ & $\underline{\mathrm{R}}^{4}$ & $\begin{array}{r}\text { Dat } \\
\text { Poir } \\
\end{array}$ \\
\hline $\begin{array}{l}46 \\
46 \\
46 \\
46 \\
46\end{array}$ & $\begin{array}{l}5.9 \\
6.6 \\
4.8 \\
5.0 \\
4.7\end{array}$ & $\begin{array}{l}7.0 \\
7.3 \\
5.2 \\
6.6 \\
5.7\end{array}$ & $\begin{array}{l}8.1 \\
8.1 \\
6.1 \\
7.4 \\
6.8\end{array}$ & $\begin{array}{l}9.0 \\
9.2 \\
7.3 \\
8.5 \\
7.6\end{array}$ & $\begin{array}{r}10.7 \\
9.6 \\
7.8 \\
9.7 \\
8.5\end{array}$ & $\begin{array}{r}11.1 \\
9.6 \\
8.1 \\
11.0 \\
9.3\end{array}$ & $\begin{array}{l}5.0 \\
5.1 \\
5.6 \\
5.7 \\
5.8\end{array}$ & $\begin{array}{l}1.04 \\
1.05 \\
1.21 \\
1.23 \\
1.30\end{array}$ & 2 \\
\hline $\begin{array}{l}46 \\
46 \\
46 \\
46 \\
46\end{array}$ & $\begin{array}{l}6.0 \\
4.8 \\
3.5 \\
5.2 \\
6.0\end{array}$ & $\begin{array}{l}6.4 \\
6.8 \\
5.2 \\
6.3 \\
7.2\end{array}$ & $\begin{array}{l}7.3 \\
7.8 \\
6.8 \\
7.1 \\
9.6\end{array}$ & $\begin{array}{r}8.4 \\
8.2 \\
7.6 \\
7.7 \\
10.5\end{array}$ & $\begin{array}{r}8.9 \\
10.0 \\
8.2 \\
8.0 \\
11.3\end{array}$ & $\begin{array}{r}9.3 \\
11.6 \\
8.7 \\
9.1 \\
12.2\end{array}$ & $\begin{array}{l}6.1 \\
6.3 \\
6.4 \\
6.5 \\
7.1\end{array}$ & $\begin{array}{l}1.36 \\
1.42 \\
1.46 \\
1.49 \\
1.73\end{array}$ & \\
\hline $\begin{array}{l}46 \\
47 \\
47 \\
47 \\
47\end{array}$ & $\begin{array}{r}4.8 \\
- \\
- \\
-\end{array}$ & $\begin{array}{r}6.2 \\
- \\
- \\
- \\
-\end{array}$ & $\begin{array}{r}7.0 \\
--- \\
--- \\
---\end{array}$ & $\begin{array}{r}7.7 \\
- \\
- \\
- \\
-\end{array}$ & $\begin{array}{r}8.4 \\
--- \\
---- \\
----\end{array}$ & $\begin{array}{r}8.6 \\
14.0 \\
23.0 \\
21.0 \\
28.0\end{array}$ & $\begin{array}{r}7.3 \\
--- \\
--\end{array}$ & $\begin{array}{l}1.80 \\
0.30 \\
0.34 \\
0.40 \\
0.41\end{array}$ & 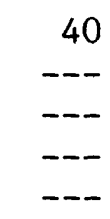 \\
\hline $\begin{array}{l}47 \\
47 \\
47 \\
47 \\
47\end{array}$ & $\begin{array}{l}--- \\
-- \\
--- \\
---\end{array}$ & $\begin{array}{l}--- \\
--- \\
--- \\
--- \\
---\end{array}$ & $\begin{array}{l}--- \\
--- \\
--- \\
--- \\
---\end{array}$ & $\begin{array}{l}--- \\
--- \\
--- \\
--- \\
---\end{array}$ & $\begin{array}{l}--- \\
---- \\
---- \\
---- \\
----\end{array}$ & $\begin{array}{l}28.6 \\
27.0 \\
31.0 \\
32.0 \\
35.0\end{array}$ & $\begin{array}{l}--- \\
---- \\
---- \\
---- \\
----\end{array}$ & $\begin{array}{l}0.42 \\
0.45 \\
0.47 \\
0.53 \\
0.55\end{array}$ & $\begin{array}{c}-- \\
-- \\
-- \\
-- \\
--\end{array}$ \\
\hline $\begin{array}{l}47 \\
47 \\
47 \\
47 \\
47\end{array}$ & $\begin{array}{l}--- \\
--- \\
-- \\
--\end{array}$ & $\begin{array}{l}-- \\
--- \\
--- \\
--\end{array}$ & $\begin{array}{l}--- \\
---- \\
---- \\
---- \\
---\end{array}$ & $\begin{array}{l}--- \\
---- \\
---- \\
---- \\
----\end{array}$ & $\begin{array}{l}--- \\
--- \\
---- \\
---- \\
----\end{array}$ & $\begin{array}{l}28.0 \\
14.0 \\
17.0 \\
14.0 \\
13.0\end{array}$ & $\begin{array}{l}--- \\
--- \\
--- \\
--- \\
----\end{array}$ & $\begin{array}{l}0.56 \\
0.58 \\
0.60 \\
0.64 \\
0.65\end{array}$ & $\begin{array}{c}-- \\
-- \\
-- \\
-- \\
--\end{array}$ \\
\hline $\begin{array}{l}47 \\
47 \\
47 \\
47 \\
47\end{array}$ & $\begin{array}{l}-- \\
- \\
-- \\
-- \\
-\end{array}$ & $\begin{array}{l}-- \\
-\cdots \\
--- \\
--- \\
--\end{array}$ & $\begin{array}{l}--- \\
---- \\
---- \\
---- \\
----\end{array}$ & $\begin{array}{l}--- \\
--- \\
--- \\
--- \\
---\end{array}$ & $\begin{array}{l}--- \\
--- \\
--- \\
--- \\
--\end{array}$ & $\begin{array}{r}9.0 \\
13.0 \\
14.0 \\
8.0 \\
7.0\end{array}$ & $\begin{array}{l}--- \\
--- \\
--- \\
--- \\
---\end{array}$ & $\begin{array}{l}0.68 \\
0.70 \\
0.72 \\
0.80 \\
0.82\end{array}$ & $\begin{array}{c}-- \\
-- \\
-- \\
--\end{array}$ \\
\hline $\begin{array}{l}47 \\
47 \\
48 \\
48 \\
48\end{array}$ & $\begin{array}{r}10.5 \\
5.7 \\
3.8\end{array}$ & $\begin{array}{r}11.5 \\
8.2 \\
4.9\end{array}$ & $\begin{array}{r}13.1 \\
11.4 \\
6.3\end{array}$ & $\begin{array}{r}14.0 \\
14.0 \\
8.1\end{array}$ & $\begin{array}{r}15.4 \\
16.8 \\
9.8\end{array}$ & $\begin{array}{l}18.0 \\
13.5 \\
17.3 \\
21.8 \\
13.9\end{array}$ & $\begin{array}{r}7.2 \\
8.1 \\
10.0\end{array}$ & $\begin{array}{l}1.60 \\
2.00 \\
0.52 \\
0.58 \\
0.76\end{array}$ & $\begin{array}{r}43 \\
225 \\
364\end{array}$ \\
\hline
\end{tabular}


TABLE 2A. LIST OF ANADARKO BASIN VELLS FROM VHICH LOG POROSITY DATA UERE OBTAINED. [ ${ }^{1}$ Well number refers to table 2B; ${ }^{2}$ location (section, township, and range), operator, and well name are as shown on well-log headers.]

$\underline{\text { Well }}^{1} \quad \underline{\text { Location }}^{2}$

Sec. 21, T.8N., R. 12W. Sec. 1, T.7N., R.12W.

Sec. 24, T. 10N. , R. 13W.

Sec. 32, T.8N., R. $9 \mathrm{~W}$.

Sec.29, T.7N., R. 9W.

Sec. 25, T.7N., R. 11W.

Sec.18,T.9N.,R.13W.

Sec.19, T.10N., R.13W.

Sec. 10, T. 8 N. , R.13W.

Sec. 6, T. 7N. , R. 9W.

Sec. 18, T.8N. ,R.9W.

Sec. 28, T. 8N. , R.11W.

Sec. 33, T. 8N. , R.10W.

Sec.13, T. 7N. , R. 10W.

Sec. 26, T.7N., R.9W.

Sec. 4, T. 7N. , R. 11W.

Sec. 10, T. 7N. , R.9W.

Sec. 26, T. 7N. , R. 10W.

Sec. 19, T.11N., R.13W.

Sec. 25, T.11N., R. 12W.

Sec. 10, T.16N., R. 12W.

Sec. 7, T. 16N., R.10W.

Sec. 4,T.18N. , R.11W.

Sec. 34, T.22N., R. 9W.

Sec. 3, T. 21N., R. 9W.

Sec.19,T.20N. , R. 9W.

Sec.16,T.21N., R.11W.

Sec. 31, T. 20N. , R. 13W.

Sec. 25, T. 20N. , R. 10W.

Sec. 36, T.20N., R. 10W.

Sec. 28, T. 20N., R.10W.

Sec. 21, T.22N. , R. 16W.

Sec.16, T.20N., R.16W.
Operator $^{2}$

Sohio Petroleum

Sohio Petroleum

Helmerich and Payne

Sohio Petroleum

Shell 0 il

Helmerich and Payne

L.G. Williams Inc

Hadson Petroleum Corp

Dyco Petroleum Corp

Cotton Petroleum Corp

Cotton Petroleum Corp

GHK

Sanguine LTD

Shell 0 il

Sanguine LTD

Sohio Petroleum

Cotton Petroleum Corp

Davis 0il

Lear Pet. Expl. Inc

Cotton Petroleum Corp

Davis 0il

Bogert 0 il

Bogert 0il

Arapaho Petroleum

Berry Petroleum

Western Pacific Pet.

Ladd Petroleum Corp

Nobel Operating Inc

Bogert 0il

Cuesta Energy Corp

Prime Energy Corp

Shell 0il

TXO Production Corp
Well Name ${ }^{2}$

1-21 Stockton

1-1 Cay

1 Phifer

1-32 Harper

1-29 Bruer

1-25 Charles Adams

1-18 Allred

1-19 Adams

1-10 Moses Caley

1 Mary

1-A Cox

1-28 Didier

1 Griffitis

1-13 Moore

1 Mae West

1-4 Nikkel

1-10 Kvasnica

1-26 J.D. Miles

1-19 Horn

1-A Dorsey

1 Pickett

1-7 Bernhard t

1-4 Henry

2-34 Cottons

1-3 Perry

1-1 Patterson

4 Shiddell

2 Sholters

1-25 Frank

1-36 Seelke

1-28 Bierig

2-21 Foster

1-A Hoskin 
Table 2B. ANADARKO BASIN VELL-LOG POROSITY DATA

[ ${ }^{1}$ Well number from table $2 \mathrm{~A} ;{ }^{2} \mathrm{ft} ;{ }^{3}$ thickness of data interval ( $\mathrm{ft}$ ); ${ }^{4} \mathrm{log}-$ derived porosity (\%); 5 equivalent vitrinite reflectance (\%); 6 age code (1=Lower Paleozoic, 2=Pennsylvanian, 3=Permian).]

\begin{tabular}{|c|c|c|c|c|c|c|c|c|c|c|c|}
\hline $\mathrm{We}^{1}{ }^{1}$ & Depth ${ }^{2}$ & $\mathrm{Dz}^{3}$ & $\mathrm{POR}^{4}$ & $\underline{R}_{0}^{5}$ & $\underline{A G}^{6}$ & $\mathrm{Well}^{1}$ & Depth $^{2}$ & $\mathrm{DZ}^{3}$ & $\mathrm{POR}^{4}$ & $\underline{R}_{0}^{5}$ & $\underline{A G}^{6}$ \\
\hline 1 & 7531 & 5 & 11.0 & 0.77 & 2 & 2 & 10956 & 8 & 4.2 & 1.07 & 2 \\
\hline 1 & 7608 & 6 & 2.8 & 0.78 & 2 & 2 & 10988 & 18 & 2.0 & 1.08 & 2 \\
\hline 1 & 8658 & 12 & 6.0 & 0.86 & 2 & 2 & 11196 & 16 & 6.0 & 1.10 & 2 \\
\hline 1 & 8672 & 4 & 5.6 & 0.86 & 2 & 2 & 11982 & 8 & 6.0 & 1.19 & 2 \\
\hline 1 & 8696 & 4 & 6.0 & 0.86 & 2 & 2 & 12048 & 4 & 4.5 & 1.19 & 2 \\
\hline 1 & 8700 & 4 & 7.6 & 0.86 & 2 & 2 & 13338 & 5 & 5.1 & 1.35 & 2 \\
\hline 1 & 8704 & 12 & 6.2 & 0.86 & 2 & 2 & 18626 & 16 & 4.8 & 2.27 & 2 \\
\hline 1 & 8716 & 8 & 6.8 & 0.86 & 2 & 2 & 18642 & 8 & 4.1 & 2.27 & 2 \\
\hline 1 & 8736 & 10 & 6.0 & 0.87 & 2 & 2 & 18686 & 14 & 6.3 & 2.28 & 2 \\
\hline 1 & 8746 & 8 & 5.2 & 0.87 & 2 & 2 & 18726 & 4 & 2.6 & 2.29 & 2 \\
\hline 1 & 8754 & 10 & 6.0 & 0.87 & 2 & 2 & 18950 & 10 & 4.4 & 2.34 & 2 \\
\hline 1 & 8770 & 6 & 5.8 & 0.87 & 2 & 2 & 18966 & 8 & 3.8 & 2.34 & 2 \\
\hline 1 & 9586 & 6 & 5.0 & 0.94 & 2 & 2 & 18977 & 7 & 3.0 & 2.34 & 2 \\
\hline 1 & 9595 & 5 & 6.0 & 0.94 & 2 & 2 & 18986 & 12 & 7.3 & 2.35 & 2 \\
\hline 1 & 11056 & 12 & 4.5 & 1.08 & 2 & 2 & 19080 & 12 & 3.0 & 2.37 & 2 \\
\hline 1 & 11068 & 4 & 8.0 & 1.09 & 2 & 2 & 19099 & 4 & 3.4 & 2.37 & 2 \\
\hline 1 & 11074 & 6 & 6.2 & 1.09 & 2 & 2 & 19102 & 6 & 4.7 & 2.37 & 2 \\
\hline 1 & 12668 & 4 & 5.2 & 1.27 & 2 & 2 & 19108 & 12 & 3.0 & 2.37 & 2 \\
\hline 1 & 12672 & 8 & 7.3 & 1.27 & 2 & 2 & 19120 & 8 & 4.2 & 2.38 & 2 \\
\hline 1 & 12698 & 12 & 7.3 & 1.27 & 2 & 2 & 19128 & 10 & 4.6 & 2.38 & 2 \\
\hline 1 & 15062 & 4 & 6.8 & 1.60 & 2 & 2 & 19736 & 8 & 4.8 & 2.52 & 2 \\
\hline 1 & 18792 & 4 & 3.0 & 2.30 & 2 & 2 & 19750 & 4 & 5.0 & 2.53 & 2 \\
\hline 1 & 18800 & 4 & 3.0 & 2.30 & 2 & 2 & 19754 & 4 & 4.3 & 2.53 & 2 \\
\hline 1 & 19116 & 6 & 3.0 & 2.38 & 2 & 2 & 20280 & 4 & 4.0 & 2.66 & 2 \\
\hline 1 & 19618 & 10 & 8.0 & 2.50 & 2 & 2 & 20298 & 4 & 4.8 & 2.67 & 2 \\
\hline 1 & 19628 & 8 & 6.5 & 2.50 & 2 & 3 & 5136 & 10 & 18.1 & 0.61 & 4 \\
\hline 1 & 20806 & 6 & 4.2 & 2.80 & 2 & 3 & 5182 & 6 & 17.0 & 0.61 & 4 \\
\hline 1 & 20852 & 6 & 5.1 & 2.81 & 2 & 3 & 5294 & 16 & 17.1 & 0.62 & 4 \\
\hline 1 & 20866 & 6 & 7.9 & 2.82 & 2 & 3 & 5364 & 10 & 17.2 & 0.62 & 4 \\
\hline 2 & 5454 & 12 & 24.8 & 0.63 & 2 & 3 & 6190 & 12 & 16.0 & 0.68 & 2 \\
\hline 2 & 6003 & 4 & 8.3 & 0.66 & 2 & 3 & 6410 & 4 & 13.7 & 0.69 & 2 \\
\hline 2 & 7246 & 12 & 11.5 & 0.75 & 2 & 3 & 6424 & 10 & 14.8 & 0.69 & 2 \\
\hline 2 & 7894 & 8 & 13.1 & 0.80 & 2 & 3 & 6442 & 6 & 15.0 & 0.69 & 2 \\
\hline 2 & 8930 & 4 & 5.3 & 0.88 & 2 & 3 & 6448 & 6 & 11.8 & 0.69 & 2 \\
\hline 2 & 10950 & 6 & 2.5 & 1.07 & 2 & 3 & 7176 & 8 & 11.9 & 0.74 & 2 \\
\hline
\end{tabular}


Table 2B. (continued)

\begin{tabular}{|c|c|c|c|c|c|c|c|c|c|c|c|}
\hline $\mathrm{Well}^{1}$ & Depth $^{2}$ & $\underline{\mathrm{DZ}}^{3}$ & $\underline{P O R}^{4}$ & $\underline{R}_{\theta}^{5}$ & $\underline{A G}^{6}$ & Well $^{1}$ & Depth ${ }^{2}$ & $\mathrm{DZ}^{3}$ & $\mathrm{POR}^{4}$ & $\underline{R}_{\theta}^{5}$ & $\underline{A G}^{6}$ \\
\hline 3 & 7184 & 12 & 13.5 & 0.74 & 2 & 4 & 8818 & 10 & 7.2 & 0.87 & 2 \\
\hline 3 & 8420 & 10 & 4.9 & 0.84 & 2 & 4 & 8828 & 10 & 4.9 & 0.87 & 2 \\
\hline 3 & 8436 & 8 & 5.1 & 0.84 & 2 & 4 & 9118 & 4 & 12.0 & 0.90 & 2 \\
\hline 3 & 9586 & 10 & 6.0 & 0.94 & 2 & 4 & 9658 & 34 & 6.5 & 0.95 & 2 \\
\hline 3 & 10442 & 10 & 1.5 & 1.02 & 2 & 4 & 9696 & 4 & 4.2 & 0.95 & 2 \\
\hline 3 & 10488 & 4 & 3.5 & 1.03 & 2 & 4 & 9704 & 8 & 4.2 & 0.95 & 2 \\
\hline 3 & 10508 & 12 & 7.6 & 1.03 & 2 & 4 & 11298 & 4 & 2.5 & 1.11 & 2 \\
\hline 3 & 10520 & 16 & 6.7 & 1.03 & 2 & 4 & 11966 & 8 & 4.0 & 1.19 & 2 \\
\hline 3 & 11410 & 6 & 6.3 & 1.12 & 2 & 4 & 12020 & 12 & 4.2 & 1.19 & 2 \\
\hline 3 & 12162 & 6 & 7.1 & 1.21 & 2 & 4 & 12034 & 4 & 3.3 & 1.19 & 2 \\
\hline 3 & 15190 & 6 & 7.2 & 1.90 & 3 & 4 & 12460 & 4 & 6.0 & 1.24 & 2 \\
\hline 3 & 15444 & 4 & 3.8 & 1.93 & 3 & 4 & 12478 & 12 & 5.1 & 1.25 & 2 \\
\hline 3 & 15476 & 8 & 4.3 & 1.93 & 3 & 4 & 12492 & 4 & 7.0 & 1.25 & 2 \\
\hline 3 & 19186 & 10 & 5.0 & 2.39 & 1 & 4 & 12556 & 10 & 6.0 & 1.26 & 2 \\
\hline 3 & 19196 & 10 & 4.0 & 2.40 & 1 & 4 & 12566 & 8 & 5.1 & 1.26 & 2 \\
\hline 3 & 19210 & 4 & 5.6 & 2.40 & 1 & 4 & 12574 & 4 & 8.6 & 1.26 & 2 \\
\hline 3 & 19214 & 8 & 4.0 & 2.40 & 1 & 4 & 12700 & 6 & 4.8 & 1.27 & 2 \\
\hline 3 & 19224 & 6 & 4.1 & 2.40 & 1 & 4 & 12870 & 4 & 4.5 & 1.29 & 2 \\
\hline 4 & 4280 & 4 & 21.8 & 0.56 & 4 & 4 & 14640 & 6 & 11.3 & 1.54 & 2 \\
\hline 4 & 4350 & 8 & 23.9 & 0.56 & 4 & 4 & 16624 & 12 & 9.0 & 1.86 & 2 \\
\hline 4 & 4362 & 6 & 23.0 & 0.57 & 4 & 4 & 16636 & 16 & 7.3 & 1.87 & 2 \\
\hline 4 & 4496 & 6 & 20.6 & 0.57 & 4 & 5 & 4172 & 4 & 24.9 & 0.56 & 4 \\
\hline 4 & 4603 & 5 & 22.8 & 0.58 & 4 & 5 & 4406 & 8 & 24.1 & 0.57 & 4 \\
\hline 4 & 4704 & 4 & 20.8 & 0.58 & 4 & 5 & 4948 & 16 & 23.1 & 0.60 & 4 \\
\hline 4 & 5132 & 8 & 20.1 & 0.61 & 4 & 5 & 4994 & 6 & 24.2 & 0.60 & 4 \\
\hline 4 & 6354 & 4 & 17.8 & 0.69 & 2 & 5 & 5330 & 6 & 20.6 & 0.62 & 4 \\
\hline 4 & 6424 & 4 & 16.7 & 0.69 & 2 & 5 & 5398 & 4 & 21.8 & 0.63 & 4 \\
\hline 4 & 6434 & 4 & 16.6 & 0.69 & 2 & 5 & 5530 & 12 & 21.5 & 0.63 & 2 \\
\hline 4 & 6732 & 14 & 18.0 & 0.71 & 2 & 5 & 5716 & 4 & 22.8 & 0.65 & 2 \\
\hline 4 & 7118 & 10 & 16.9 & 0.74 & 2 & 5 & 5810 & 8 & 21.8 & 0.65 & 2 \\
\hline 4 & 7130 & 4 & 12.5 & 0.74 & 2 & 5 & 5842 & 6 & 19.3 & 0.65 & 2 \\
\hline 4 & 7502 & 4 & 11.5 & 0.77 & 2 & 5 & 5930 & 4 & 17.8 & 0.66 & 2 \\
\hline 4 & 7650 & 16 & 15.1 & 0.78 & 2 & 5 & 5944 & 4 & 18.7 & 0.66 & 2 \\
\hline 4 & 7751 & 13 & 13.5 & 0.79 & 2 & 5 & 5950 & 10 & 16.0 & 0.66 & 2 \\
\hline 4 & 8570 & 4 & 9.0 & 0.85 & 2 & 5 & 6028 & 12 & 17.2 & 0.67 & 2 \\
\hline
\end{tabular}


Table 2B. (continued)

\begin{tabular}{|c|c|c|c|c|c|c|c|c|c|c|c|}
\hline$\underline{\text { Well }}^{1}$ & Depth $^{2}$ & $\underline{\mathrm{Dz}}^{3}$ & $\underline{P O R}^{4}$ & $\underline{R}_{\theta}^{5}$ & $\underline{A G}^{6}$ & $\mathrm{Well}^{1}$ & Depth $^{2}$ & $\mathrm{Dz}^{3}$ & $\underline{P O R}^{4}$ & $\underline{R}_{\theta}^{5}$ & $\underline{A G}^{6}$ \\
\hline 5 & 6054 & 4 & 19.2 & 0.67 & 2 & 5 & 9668 & 4 & 9.2 & 0.95 & 2 \\
\hline 5 & 6058 & 7 & 19.9 & 0.67 & 2 & 5 & 9672 & 10 & 7.0 & 0.95 & 2 \\
\hline 5 & 6134 & 8 & 19.7 & 0.67 & 2 & 5 & 9682 & 12 & 7.0 & 0.95 & 2 \\
\hline 5 & 6156 & 6 & 19.6 & 0.67 & 2 & 5 & 9694 & 10 & 7.9 & 0.95 & 2 \\
\hline 5 & 6206 & 6 & 22.3 & 0.68 & 2 & 5 & 9708 & 6 & 7.6 & 0.95 & 2 \\
\hline 5 & 6212 & 6 & 20.0 & 0.68 & 2 & 5 & 9714 & 10 & 5.8 & 0.95 & 2 \\
\hline 5 & 6289 & 7 & 21.4 & 0.68 & 2 & 5 & 9724 & 8 & 7.6 & 0.95 & 2 \\
\hline 5 & 6408 & 8 & 19.3 & 0.69 & 2 & 5 & 9732 & 6 & 4.5 & 0.95 & 2 \\
\hline 5 & 6544 & 8 & 15.2 & 0.70 & 2 & 5 & 9746 & 10 & 5.9 & 0.95 & 2 \\
\hline 5 & 6592 & 4 & 16.9 & 0.70 & 2 & 5 & 9804 & 18 & 6.3 & 0.96 & 2 \\
\hline 5 & 6746 & 5 & 2.8 & 0.71 & 2 & 5 & 10002 & 6 & 4.4 & 0.98 & 2 \\
\hline 5 & 6784 & 10 & 15.3 & 0.72 & 2 & 5 & 10008 & 12 & 5.7 & 0.98 & 2 \\
\hline 5 & 7000 & 6 & 16.8 & 0.73 & 2 & 5 & 11137 & 4 & 2.7 & 1.09 & 2 \\
\hline 5 & 7028 & 12 & 19.3 & 0.73 & 2 & 5 & 11141 & 9 & 4.0 & 1.09 & 2 \\
\hline 5 & 7070 & 8 & 1.7 & 0.74 & 2 & 5 & 11150 & 6 & 2.2 & 1.09 & 2 \\
\hline 5 & 7384 & 4 & 8.7 & 0.76 & 2 & 5 & 11161 & 6 & 5.0 & 1.10 & 2 \\
\hline 5 & 7596 & 4 & 14.5 & 0.77 & 2 & 5 & 11167 & 5 & 2.1 & 1.10 & 2 \\
\hline 5 & 7600 & 6 & 17.3 & 0.78 & 2 & 5 & 11206 & 6 & 5.6 & 1.10 & 2 \\
\hline 5 & 7630 & 6 & 16.2 & 0.78 & 2 & 5 & 11434 & 4 & 2.0 & 1.13 & 2 \\
\hline 5 & 7636 & 4 & 12.5 & 0.78 & 2 & 5 & 11436 & 6 & 6.0 & 1.13 & 2 \\
\hline 5 & 7640 & 4 & 17.8 & 0.78 & 2 & 5 & 11450 & 10 & 3.6 & 1.13 & 2 \\
\hline 5 & 7700 & 14 & 16.2 & 0.78 & 2 & 5 & 11474 & 6 & 4.6 & 1.13 & 2 \\
\hline 5 & 7714 & 6 & 14.0 & 0.78 & 2 & 5 & 12120 & 6 & 5.0 & 1.20 & 2 \\
\hline 5 & 7720 & 10 & 16.2 & 0.78 & 2 & 5 & 12152 & 8 & 4.1 & 1.21 & 2 \\
\hline 5 & 7766 & 6 & 1.7 & 0.79 & 2 & 5 & 12194 & 6 & 2.3 & 1.21 & 2 \\
\hline 5 & 8276 & 4 & 6.7 & 0.83 & 2 & 5 & 12560 & 6 & 7.2 & 1.26 & 2 \\
\hline 5 & 8588 & 4 & 3.5 & 0.85 & 2 & 5 & 12566 & 6 & 8.3 & 1.26 & 2 \\
\hline 5 & 8592 & 14 & 8.1 & 0.85 & 2 & 5 & 12950 & 6 & 4.9 & 1.30 & 2 \\
\hline 5 & 8656 & 4 & 8.0 & 0.86 & 2 & 5 & 16748 & 6 & 4.3 & 1.89 & 2 \\
\hline 5 & 8796 & 10 & 10.8 & 0.87 & 2 & 5 & 16754 & 5 & 3.2 & 1.89 & 2 \\
\hline 5 & 8806 & 10 & 11.1 & 0.87 & 2 & 5 & 16788 & 4 & 5.9 & 1.89 & 2 \\
\hline 5 & 8818 & 6 & 13.5 & 0.87 & 2 & 5 & 16796 & 6 & 4.3 & 1.90 & 2 \\
\hline 5 & 8830 & 10 & 10.1 & 0.87 & 2 & 5 & 16866 & 6 & 5.5 & 1.91 & 2 \\
\hline 5 & 9646 & 16 & 6.8 & 0.95 & 2 & 5 & 16872 & 4 & 1.7 & 1.91 & 2 \\
\hline 5 & 9664 & 4 & 7.8 & 0.95 & 2 & 5 & 16878 & 10 & 2.1 & 1.91 & 2 \\
\hline
\end{tabular}


Table 2B. (continued)

\begin{tabular}{|c|c|c|c|c|c|c|c|c|c|c|c|}
\hline Well ${ }^{1}$ & Depth ${ }^{2}$ & $\underline{\mathrm{DZ}}^{3}$ & $\underline{\mathrm{POR}}^{4}$ & $\underline{R}_{\theta}^{5}$ & $\underline{A G}^{6}$ & $\mathrm{Well}^{1}$ & Depth $^{2}$ & $\mathrm{DZ}^{3}$ & $\mathrm{POR}^{4}$ & $\mathrm{R}_{0}^{5}$ & $\underline{A G}^{6}$ \\
\hline $\begin{array}{l}5 \\
5 \\
5 \\
5 \\
5\end{array}$ & $\begin{array}{l}16896 \\
17122 \\
17204 \\
17208 \\
17214\end{array}$ & $\begin{array}{r}10 \\
6 \\
4 \\
6 \\
4\end{array}$ & $\begin{array}{l}2.0 \\
4.0 \\
2.8 \\
6.0 \\
3.5\end{array}$ & $\begin{array}{l}1.91 \\
1.96 \\
1.97 \\
1.97 \\
1.97\end{array}$ & $\begin{array}{l}2 \\
2 \\
2 \\
2 \\
2\end{array}$ & $\begin{array}{l}6 \\
6 \\
6 \\
6 \\
6\end{array}$ & $\begin{array}{l}8144 \\
9684 \\
9692 \\
9700 \\
9714\end{array}$ & $\begin{array}{r}12 \\
8 \\
6 \\
10 \\
6\end{array}$ & $\begin{array}{r}12.0 \\
6.3 \\
3.7 \\
7.0 \\
5.3\end{array}$ & $\begin{array}{l}0.82 \\
0.95 \\
0.95 \\
0.95 \\
0.95\end{array}$ & $\begin{array}{l}2 \\
2 \\
2 \\
2 \\
2\end{array}$ \\
\hline $\begin{array}{l}5 \\
5 \\
5 \\
5 \\
5\end{array}$ & $\begin{array}{l}17218 \\
17226 \\
17228 \\
17238 \\
17326\end{array}$ & $\begin{array}{r}6 \\
4 \\
10 \\
4 \\
12\end{array}$ & $\begin{array}{l}6.7 \\
3.4 \\
4.9 \\
2.9 \\
3.8\end{array}$ & $\begin{array}{l}1.98 \\
1.98 \\
1.98 \\
1.98 \\
2.00\end{array}$ & $\begin{array}{l}2 \\
2 \\
2 \\
2 \\
2\end{array}$ & $\begin{array}{l}6 \\
6 \\
6 \\
6 \\
6\end{array}$ & $\begin{array}{r}9750 \\
9878 \\
11120 \\
11186 \\
11196\end{array}$ & $\begin{array}{r}6 \\
12 \\
10 \\
10 \\
18\end{array}$ & $\begin{array}{l}5.7 \\
4.3 \\
5.0 \\
0.5 \\
5.8\end{array}$ & $\begin{array}{l}0.96 \\
0.97 \\
1.09 \\
1.10 \\
1.10\end{array}$ & $\begin{array}{l}2 \\
2 \\
2 \\
2 \\
2\end{array}$ \\
\hline $\begin{array}{l}6 \\
6 \\
6 \\
6 \\
6\end{array}$ & $\begin{array}{l}3700 \\
4796 \\
4834 \\
4940 \\
4995\end{array}$ & $\begin{array}{r}6 \\
14 \\
12 \\
12 \\
4\end{array}$ & $\begin{array}{r}4.7 \\
23.0 \\
21.2 \\
17.1 \\
20.0\end{array}$ & $\begin{array}{l}0.53 \\
0.59 \\
0.59 \\
0.60 \\
0.60\end{array}$ & $\begin{array}{l}4 \\
2 \\
2 \\
2 \\
2\end{array}$ & $\begin{array}{l}6 \\
6 \\
6 \\
6 \\
6\end{array}$ & $\begin{array}{l}11330 \\
11338 \\
11358 \\
11376 \\
11384\end{array}$ & $\begin{array}{r}4 \\
14 \\
18 \\
8 \\
10\end{array}$ & $\begin{array}{l}2.2 \\
2.0 \\
1.6 \\
2.8 \\
8.6\end{array}$ & $\begin{array}{l}1.11 \\
1.11 \\
1.12 \\
1.12 \\
1.12\end{array}$ & $\begin{array}{l}2 \\
2 \\
2 \\
2 \\
2\end{array}$ \\
\hline $\begin{array}{l}6 \\
6 \\
6 \\
6 \\
6\end{array}$ & $\begin{array}{l}5072 \\
5420 \\
5644 \\
5748 \\
5754\end{array}$ & $\begin{array}{r}8 \\
14 \\
8 \\
6 \\
6\end{array}$ & $\begin{array}{l}18.0 \\
18.0 \\
17.1 \\
20.0 \\
20.8\end{array}$ & $\begin{array}{l}0.61 \\
0.63 \\
0.64 \\
0.65 \\
0.65\end{array}$ & $\begin{array}{l}2 \\
2 \\
2 \\
2 \\
2\end{array}$ & $\begin{array}{l}6 \\
6 \\
6 \\
6 \\
6\end{array}$ & $\begin{array}{l}11394 \\
12136 \\
12834 \\
13360 \\
14260\end{array}$ & $\begin{array}{r}14 \\
8 \\
10 \\
18 \\
16\end{array}$ & $\begin{array}{l}5.1 \\
5.6 \\
5.2 \\
4.7 \\
5.1\end{array}$ & $\begin{array}{l}1.12 \\
1.20 \\
1.29 \\
1.36 \\
1.48\end{array}$ & $\begin{array}{l}2 \\
2 \\
2 \\
2 \\
2\end{array}$ \\
\hline $\begin{array}{l}6 \\
6 \\
6 \\
6 \\
6\end{array}$ & $\begin{array}{l}5760 \\
6180 \\
6188 \\
6260 \\
6308\end{array}$ & $\begin{array}{r}6 \\
8 \\
8 \\
4 \\
18\end{array}$ & $\begin{array}{r}17.1 \\
18.9 \\
17.7 \\
14.0 \\
1.7\end{array}$ & $\begin{array}{l}0.65 \\
0.68 \\
0.68 \\
0.68 \\
0.68\end{array}$ & $\begin{array}{l}2 \\
2 \\
2 \\
2 \\
2\end{array}$ & $\begin{array}{l}6 \\
6 \\
6 \\
6 \\
6\end{array}$ & $\begin{array}{l}14658 \\
18242 \\
18272 \\
18364 \\
19496\end{array}$ & $\begin{array}{r}22 \\
6 \\
10 \\
14 \\
6\end{array}$ & $\begin{array}{l}5.7 \\
3.3 \\
3.3 \\
3.7 \\
3.8\end{array}$ & $\begin{array}{l}1.54 \\
2.18 \\
2.19 \\
2.21 \\
2.47\end{array}$ & $\begin{array}{l}2 \\
2 \\
2 \\
2 \\
2\end{array}$ \\
\hline $\begin{array}{l}6 \\
6 \\
6 \\
6 \\
6\end{array}$ & $\begin{array}{l}6916 \\
7394 \\
7430 \\
7456 \\
7462\end{array}$ & $\begin{array}{r}14 \\
6 \\
8 \\
4 \\
8\end{array}$ & $\begin{array}{l}19.2 \\
14.0 \\
12.2 \\
11.4 \\
13.0\end{array}$ & $\begin{array}{l}0.73 \\
0.76 \\
0.76 \\
0.76 \\
0.76\end{array}$ & $\begin{array}{l}2 \\
2 \\
2 \\
2 \\
2\end{array}$ & $\begin{array}{l}6 \\
6 \\
6 \\
6 \\
6\end{array}$ & $\begin{array}{l}19588 \\
19616 \\
19628 \\
19686 \\
19700\end{array}$ & $\begin{array}{r}6 \\
8 \\
4 \\
14 \\
8\end{array}$ & $\begin{array}{l}4.0 \\
4.1 \\
5.0 \\
4.3 \\
5.6\end{array}$ & $\begin{array}{l}2.49 \\
2.49 \\
2.50 \\
2.51 \\
2.52\end{array}$ & $\begin{array}{l}2 \\
2 \\
2 \\
2 \\
2\end{array}$ \\
\hline $\begin{array}{l}6 \\
6 \\
6 \\
6 \\
6\end{array}$ & $\begin{array}{l}7470 \\
7480 \\
7492 \\
7644 \\
8126\end{array}$ & $\begin{array}{l}10 \\
10 \\
10 \\
12 \\
14\end{array}$ & $\begin{array}{l}15.1 \\
13.0 \\
12.3 \\
14.0 \\
13.7\end{array}$ & $\begin{array}{l}0.77 \\
0.77 \\
0.77 \\
0.78 \\
0.82\end{array}$ & $\begin{array}{l}2 \\
2 \\
2 \\
2 \\
2\end{array}$ & $\begin{array}{l}7 \\
7 \\
7 \\
7 \\
7\end{array}$ & $\begin{array}{l}6162 \\
6720 \\
6964 \\
6981 \\
7296\end{array}$ & $\begin{array}{r}8 \\
10 \\
10 \\
4 \\
12\end{array}$ & $\begin{array}{l}15.0 \\
12.3 \\
14.0 \\
14.2 \\
13.0\end{array}$ & $\begin{array}{l}0.67 \\
0.71 \\
0.73 \\
0.73 \\
0.75\end{array}$ & $\begin{array}{l}2 \\
2 \\
2 \\
2 \\
2\end{array}$ \\
\hline
\end{tabular}


Table 2B. (continued)

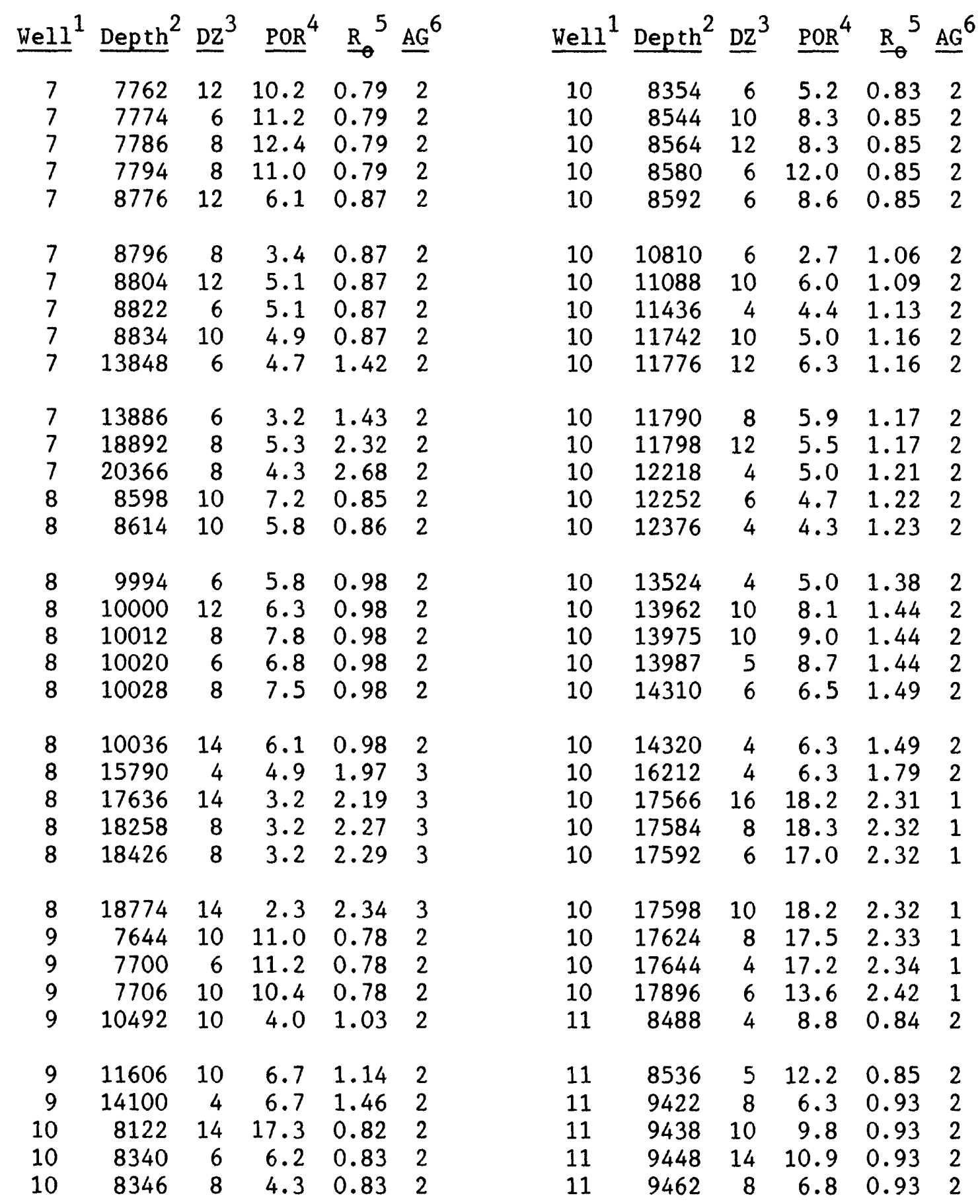


Table 2B. (continued)

\begin{tabular}{|c|c|c|c|c|c|c|c|c|c|c|c|}
\hline $\mathrm{Well}^{1}$ & Depth $^{2}$ & $\underline{\mathrm{Dz}}^{3}$ & $\mathrm{POR}^{4}$ & $\underline{R}_{0}^{5}$ & $\underline{A G}^{6}$ & $\mathrm{Well}^{1}$ & Depth $^{2}$ & $\mathrm{Dz}^{3}$ & $\underline{P O R}^{4}$ & $\underline{R}_{0}^{5}$ & $\underline{\mathrm{AG}}^{6}$ \\
\hline $\begin{array}{l}11 \\
11 \\
11 \\
11 \\
11\end{array}$ & $\begin{array}{r}9472 \\
9818 \\
10562 \\
10578 \\
11918\end{array}$ & $\begin{array}{r}4 \\
16 \\
8 \\
10 \\
8\end{array}$ & $\begin{array}{r}13.4 \\
5.0 \\
7.2 \\
5.8 \\
3.8\end{array}$ & $\begin{array}{l}0.93 \\
0.96 \\
1.03 \\
1.04 \\
1.18\end{array}$ & $\begin{array}{l}2 \\
2 \\
2 \\
2 \\
2\end{array}$ & $\begin{array}{l}12 \\
13 \\
13 \\
13 \\
13\end{array}$ & $\begin{array}{r}17842 \\
9818 \\
9834 \\
11124 \\
11140\end{array}$ & $\begin{array}{r}4 \\
8 \\
4 \\
10 \\
6\end{array}$ & $\begin{array}{r}5.5 \\
10.0 \\
11.6 \\
4.8 \\
3.7\end{array}$ & $\begin{array}{l}2.10 \\
0.96 \\
0.96 \\
1.09 \\
1.09\end{array}$ & $\begin{array}{l}2 \\
2 \\
2 \\
2 \\
2\end{array}$ \\
\hline $\begin{array}{l}11 \\
11 \\
11 \\
11 \\
11\end{array}$ & $\begin{array}{l}11928 \\
12140 \\
12144 \\
12522 \\
12546\end{array}$ & $\begin{array}{r}10 \\
4 \\
6 \\
10 \\
10\end{array}$ & $\begin{array}{l}4.5 \\
4.5 \\
7.2 \\
5.1 \\
4.7\end{array}$ & $\begin{array}{l}1.18 \\
1.21 \\
1.21 \\
1.25 \\
1.25\end{array}$ & $\begin{array}{l}2 \\
2 \\
2 \\
2 \\
2\end{array}$ & $\begin{array}{l}13 \\
13 \\
13 \\
13 \\
13\end{array}$ & $\begin{array}{l}11408 \\
12154 \\
12202 \\
12218 \\
14178\end{array}$ & $\begin{array}{r}8 \\
6 \\
10 \\
6 \\
4\end{array}$ & $\begin{array}{l}4.1 \\
4.2 \\
3.7 \\
3.7 \\
3.0\end{array}$ & $\begin{array}{l}1.12 \\
1.21 \\
1.21 \\
1.21 \\
1.47\end{array}$ & $\begin{array}{l}2 \\
2 \\
2 \\
2 \\
2\end{array}$ \\
\hline $\begin{array}{l}11 \\
11 \\
11 \\
11 \\
11\end{array}$ & $\begin{array}{l}12566 \\
12974 \\
14220 \\
15520 \\
15528\end{array}$ & $\begin{array}{r}4 \\
4 \\
4 \\
8 \\
10\end{array}$ & $\begin{array}{r}5.1 \\
6.7 \\
4.9 \\
10.8 \\
9.3\end{array}$ & $\begin{array}{l}1.26 \\
1.31 \\
1.48 \\
1.67 \\
1.68\end{array}$ & $\begin{array}{l}2 \\
2 \\
2 \\
2 \\
2\end{array}$ & $\begin{array}{l}13 \\
13 \\
13 \\
13 \\
13\end{array}$ & $\begin{array}{l}16716 \\
16742 \\
16864 \\
16904 \\
16912\end{array}$ & $\begin{array}{r}4 \\
13 \\
6 \\
8 \\
12\end{array}$ & $\begin{array}{l}5.7 \\
5.4 \\
4.2 \\
4.2 \\
3.0\end{array}$ & $\begin{array}{l}1.88 \\
1.89 \\
1.91 \\
1.92 \\
1.92\end{array}$ & $\begin{array}{l}2 \\
2 \\
2 \\
2 \\
2\end{array}$ \\
\hline $\begin{array}{l}11 \\
11 \\
11 \\
11 \\
11\end{array}$ & $\begin{array}{l}15580 \\
15596 \\
15650 \\
15662 \\
15744\end{array}$ & $\begin{array}{r}6 \\
6 \\
6 \\
14 \\
8\end{array}$ & $\begin{array}{l}7.0 \\
9.4 \\
5.0 \\
4.5 \\
5.0\end{array}$ & $\begin{array}{l}1.68 \\
1.69 \\
1.70 \\
1.70 \\
1.71\end{array}$ & $\begin{array}{l}2 \\
2 \\
2 \\
2 \\
2\end{array}$ & $\begin{array}{l}13 \\
13 \\
13 \\
13 \\
13\end{array}$ & $\begin{array}{l}17096 \\
17152 \\
17166 \\
17174 \\
17776\end{array}$ & $\begin{array}{r}18 \\
14 \\
6 \\
10 \\
4\end{array}$ & $\begin{array}{l}4.1 \\
3.0 \\
5.7 \\
3.7 \\
5.1\end{array}$ & $\begin{array}{l}1.95 \\
1.96 \\
1.97 \\
1.97 \\
2.09\end{array}$ & $\begin{array}{l}2 \\
2 \\
2 \\
2 \\
2\end{array}$ \\
\hline $\begin{array}{l}11 \\
11 \\
11 \\
11 \\
11\end{array}$ & $\begin{array}{l}15986 \\
17040 \\
17052 \\
17080 \\
17094\end{array}$ & $\begin{array}{r}12 \\
6 \\
16 \\
14 \\
18\end{array}$ & $\begin{array}{r}6.3 \\
17.0 \\
17.2 \\
17.0 \\
17.0\end{array}$ & $\begin{array}{l}1.75 \\
2.15 \\
2.16 \\
2.17 \\
2.17\end{array}$ & $\begin{array}{l}2 \\
1 \\
1 \\
1 \\
1\end{array}$ & $\begin{array}{l}13 \\
13 \\
14 \\
14 \\
14\end{array}$ & $\begin{array}{r}17780 \\
17808 \\
4118 \\
4438 \\
4534\end{array}$ & $\begin{array}{l}6 \\
5 \\
8 \\
8 \\
6\end{array}$ & $\begin{array}{r}5.3 \\
5.0 \\
23.2 \\
22.2 \\
22.6\end{array}$ & $\begin{array}{l}2.09 \\
2.09 \\
0.55 \\
0.57 \\
0.58\end{array}$ & $\begin{array}{l}2 \\
2 \\
4 \\
4 \\
4\end{array}$ \\
\hline $\begin{array}{l}11 \\
12 \\
12 \\
12 \\
12\end{array}$ & $\begin{array}{l}17334 \\
16595 \\
17333 \\
17339 \\
17350\end{array}$ & $\begin{array}{r}12 \\
5 \\
6 \\
5 \\
8\end{array}$ & $\begin{array}{r}16.0 \\
3.0 \\
6.8 \\
4.9 \\
3.5\end{array}$ & $\begin{array}{l}2.24 \\
1.86 \\
2.00 \\
2.00 \\
2.00\end{array}$ & $\begin{array}{l}1 \\
2 \\
2 \\
2 \\
2\end{array}$ & $\begin{array}{l}14 \\
14 \\
14 \\
14 \\
14\end{array}$ & $\begin{array}{l}4934 \\
4963 \\
4974 \\
5354 \\
5896\end{array}$ & $\begin{array}{r}8 \\
7 \\
5 \\
18 \\
4\end{array}$ & $\begin{array}{l}21.4 \\
21.8 \\
22.2 \\
20.3 \\
18.0\end{array}$ & $\begin{array}{l}0.60 \\
0.60 \\
0.60 \\
0.62 \\
0.66\end{array}$ & $\begin{array}{l}4 \\
4 \\
4 \\
2 \\
2\end{array}$ \\
\hline $\begin{array}{l}12 \\
12 \\
12 \\
12 \\
12\end{array}$ & $\begin{array}{l}17372 \\
17380 \\
17408 \\
17538 \\
17824\end{array}$ & $\begin{array}{l}5 \\
4 \\
4 \\
8 \\
4\end{array}$ & $\begin{array}{l}3.3 \\
3.7 \\
3.0 \\
2.4 \\
4.7\end{array}$ & $\begin{array}{l}2.01 \\
2.01 \\
2.01 \\
2.04 \\
2.10\end{array}$ & $\begin{array}{l}2 \\
2 \\
2 \\
2 \\
2\end{array}$ & $\begin{array}{l}14 \\
14 \\
14 \\
14 \\
14\end{array}$ & $\begin{array}{l}6022 \\
6116 \\
6238 \\
6248 \\
6256\end{array}$ & $\begin{array}{l}6 \\
8 \\
6 \\
4 \\
6\end{array}$ & $\begin{array}{l}17.2 \\
18.8 \\
19.5 \\
19.2 \\
18.0\end{array}$ & $\begin{array}{l}0.66 \\
0.67 \\
0.68 \\
0.68 \\
0.68\end{array}$ & $\begin{array}{l}2 \\
2 \\
2 \\
2 \\
2\end{array}$ \\
\hline
\end{tabular}


Table 2B. (continued)

\begin{tabular}{|c|c|c|c|c|c|c|c|c|c|c|c|}
\hline $\mathrm{Well}^{1}$ & Depth $^{2}$ & $\underline{\mathrm{Dz}}^{3}$ & $\mathrm{POR}^{4}$ & $\underline{R}_{0}^{5}$ & $\underline{\mathrm{AG}^{6}}$ & $\mathrm{Well}^{1}$ & Depth $^{2}$ & $\underline{D z}^{3}$ & $\mathrm{POR}^{4}$ & $\underline{R}_{0}^{5}$ & $\underline{\mathrm{AG}}^{6}$ \\
\hline 14 & 6306 & 16 & 2.2 & 0.68 & 2 & 14 & 16752 & 6 & 1.9 & 1.89 & 2 \\
\hline 14 & 6542 & 8 & 17.9 & 0.70 & 2 & 14 & 16762 & 10 & 2.7 & 1.89 & 2 \\
\hline 14 & 6998 & 8 & 16.1 & 0.73 & 2 & 14 & 16778 & 6 & 2.6 & 1.89 & 2 \\
\hline 14 & 7360 & 8 & 13.9 & 0.76 & 2 & 14 & 17020 & 6 & 3.2 & 1.94 & 2 \\
\hline 14 & 7372 & 4 & 13.9 & 0.76 & 2 & 14 & 17036 & 5 & 3.2 & 1.94 & 2 \\
\hline 14 & 7542 & 10 & 13.1 & 0.77 & 2 & 14 & 17041 & 17 & 3.0 & 1.94 & 2 \\
\hline 14 & 7572 & 6 & 11.3 & 0.77 & 2 & 14 & 17062 & 8 & 8.1 & 1.95 & 2 \\
\hline 14 & 7644 & 20 & 14.0 & 0.78 & 2 & 14 & 17070 & 4 & 5.0 & 1.95 & 2 \\
\hline 14 & 7668 & 6 & 15.8 & 0.78 & 2 & 14 & 17882 & 12 & 3.7 & 2.11 & 1 \\
\hline 14 & 8554 & 6 & 6.7 & 0.85 & 2 & 14 & 18740 & 8 & 16.2 & 2.70 & 1 \\
\hline 14 & 8702 & 6 & 7.0 & 0.86 & 2 & 14 & 18753 & 7 & 17.9 & 2.71 & 1 \\
\hline 14 & 8712 & 16 & 7.2 & 0.86 & 2 & 14 & 18760 & 14 & 17.0 & 2.71 & 1 \\
\hline 14 & 8734 & 22 & 8.3 & 0.87 & 2 & 14 & 18778 & 14 & 15.5 & 2.72 & 1 \\
\hline 14 & 8760 & 10 & 8.9 & 0.87 & 2 & 15 & 7798 & 14 & 15.9 & 0.79 & 2 \\
\hline 14 & 8770 & 8 & 7.0 & 0.87 & 2 & 15 & 7812 & 10 & 18.5 & 0.79 & 2 \\
\hline 14 & 11044 & 6 & 4.2 & 1.08 & 2 & 15 & 7824 & 16 & 17.0 & 0.79 & 2 \\
\hline 14 & 11054 & 6 & 6.0 & 1.08 & 2 & 15 & 8452 & 10 & 17.1 & 0.84 & 2 \\
\hline 14 & 11062 & 12 & 4.0 & 1.09 & 2 & 15 & 8878 & 4 & 8.8 & 0.88 & 2 \\
\hline 14 & 12020 & 6 & 9.4 & 1.19 & 2 & 15 & 8900 & 6 & 8.0 & 0.88 & 2 \\
\hline 14 & 12026 & 8 & 7.2 & 1.19 & 2 & 15 & 8906 & 4 & 9.8 & 0.88 & 2 \\
\hline 14 & 12050 & 6 & 8.7 & 1.19 & 2 & 15 & 8912 & 8 & 7.3 & 0.88 & 2 \\
\hline 14 & 12070 & 10 & 7.0 & 1.20 & 2 & 15 & 8950 & 8 & 9.7 & 0.88 & 2 \\
\hline 14 & 12156 & 4 & 4.1 & 1.21 & 2 & 15 & 8958 & 16 & 8.1 & 0.88 & 2 \\
\hline 14 & 13722 & 6 & 4.0 & 1.41 & 2 & 15 & 9624 & 10 & 9.6 & 0.94 & 2 \\
\hline 14 & 13750 & 8 & 6.3 & 1.41 & 2 & 15 & 9638 & 12 & 6.8 & 0.94 & 2 \\
\hline 14 & 13770 & 4 & 4.9 & 1.41 & 2 & 15 & 9650 & 20 & 8.3 & 0.95 & 2 \\
\hline 14 & 13792 & 4 & 5.6 & 1.42 & 2 & 15 & 9670 & 6 & 6.5 & 0.95 & 2 \\
\hline 14 & 13810 & 6 & 5.5 & 1.42 & 2 & 15 & 9682 & 22 & 7.0 & 0.95 & 2 \\
\hline 14 & 13832 & 6 & 7.0 & 1.42 & 2 & 15 & 9704 & 18 & 6.1 & 0.95 & 2 \\
\hline 14 & 15586 & 8 & 5.2 & 1.69 & 2 & 15 & 9722 & 12 & 6.9 & 0.95 & 2 \\
\hline 14 & 15594 & 7 & 6.2 & 1.69 & 2 & 15 & 9734 & 16 & 5.2 & 0.95 & 2 \\
\hline 14 & 16640 & 8 & 3.9 & 1.87 & 2 & 15 & 9750 & 10 & 6.2 & 0.96 & 2 \\
\hline 14 & 16672 & 9 & 3.8 & 1.87 & 2 & 15 & 9760 & 10 & 6.8 & 0.96 & 2 \\
\hline 14 & 16694 & 6 & 4.3 & 1.88 & 2 & 15 & 9770 & 10 & 7.5 & 0.96 & 2 \\
\hline 14 & 16708 & 6 & 5.5 & 1.88 & 2 & 15 & 9836 & 10 & 6.2 & 0.96 & 2 \\
\hline
\end{tabular}


Table 2B. (continued)

\begin{tabular}{|c|c|c|c|c|c|c|c|c|c|c|c|}
\hline $\mathrm{Well}^{1}$ & $\underline{\text { Depth }}^{2}$ & $\underline{\mathrm{Dz}}^{3}$ & $\mathrm{POR}^{4}$ & $\underline{R}^{5}$ & $\underline{A G}^{6}$ & $\mathrm{Well}^{1}$ & Depth $^{2}$ & $\underline{\mathrm{Dz}}^{3}$ & $\mathrm{POR}^{4}$ & $\underline{\mathrm{R}}_{\theta}^{5}$ & $\underline{A G}^{6}$ \\
\hline 15 & 9846 & 16 & 7.0 & 0.96 & 2 & 16 & 9062 & 22 & 5.9 & 0.89 & 2 \\
\hline 15 & 9862 & 8 & 7.7 & 0.97 & 2 & 16 & 9090 & 22 & 6.0 & 0.90 & 2 \\
\hline 15 & 9870 & 12 & 5.5 & 0.97 & 2 & 16 & 9174 & 6 & 4.5 & 0.90 & 2 \\
\hline 15 & 10674 & 20 & 7.0 & 1.05 & 2 & 16 & 11286 & 12 & 9.2 & 1.11 & 2 \\
\hline 15 & 11241 & 5 & 3.9 & 1.10 & 2 & 16 & 11694 & 8 & 6.5 & 1.15 & 2 \\
\hline 15 & 11476 & 10 & 5.8 & 1.13 & 2 & 16 & 11718 & 8 & 6.5 & 1.16 & 2 \\
\hline 15 & 12160 & 14 & 6.0 & 1.21 & 2 & 16 & 12274 & 6 & 5.0 & 1.22 & 2 \\
\hline 15 & 12180 & 12 & 7.1 & 1.21 & 2 & 16 & 12502 & 4 & 7.1 & 1.25 & 2 \\
\hline 15 & 12566 & 8 & 6.3 & 1.26 & 2 & 16 & 17120 & 4 & 3.1 & 1.96 & 2 \\
\hline 15 & 15829 & 11 & 6.7 & 1.73 & 2 & 16 & 18296 & 9 & 4.5 & 2.19 & 2 \\
\hline 15 & 16448 & 14 & 5.7 & 1.83 & 2 & 17 & 8326 & 12 & 4.0 & 0.83 & 2 \\
\hline 15 & 16508 & 10 & 4.2 & 1.84 & 2 & 17 & 8558 & 6 & 7.0 & 0.85 & 2 \\
\hline 15 & 16518 & 16 & 6.2 & 1.85 & 2 & 17 & 8578 & 4 & 11.7 & 0.85 & 2 \\
\hline 15 & 16784 & 8 & 6.2 & 1.89 & 2 & 17 & 8584 & 6 & 15.7 & 0.85 & 2 \\
\hline 15 & 16834 & 18 & 5.5 & 1.90 & 2 & 17 & 8598 & 6 & 11.9 & 0.85 & 2 \\
\hline 15 & 16856 & 16 & 6.2 & 1.91 & 2 & 17 & 8608 & 8 & 13.0 & 0.85 & 2 \\
\hline 15 & 16944 & 4 & 6.0 & 1.92 & 2 & 17 & 8864 & 16 & 6.7 & 0.88 & 2 \\
\hline 15 & 16972 & 8 & 4.8 & 1.93 & 2 & 17 & 8880 & 6 & 8.5 & 0.88 & 2 \\
\hline 15 & 16984 & 6 & 3.8 & 1.93 & 2 & 17 & 8886 & 14 & 8.8 & 0.88 & 2 \\
\hline 15 & 17382 & 8 & 8.3 & 2.01 & 2 & 17 & 8900 & 8 & 9.9 & 0.88 & 2 \\
\hline 15 & 17686 & 4 & 9.2 & 2.07 & 2 & 17 & 9276 & 6 & 6.8 & 0.91 & 2 \\
\hline 15 & 18012 & 10 & 8.0 & 2.13 & 2 & 17 & 9282 & 14 & 7.7 & 0.91 & 2 \\
\hline 15 & 18046 & 10 & 5.4 & 2.14 & 2 & 17 & 9296 & 6 & 7.6 & 0.91 & 2 \\
\hline 15 & 18082 & 4 & 4.8 & 2.15 & 2 & 17 & 9316 & 12 & 7.0 & 0.92 & 2 \\
\hline 15 & 18090 & 8 & 5.5 & 2.15 & 2 & 17 & 9328 & 22 & 6.5 & 0.92 & 2 \\
\hline 15 & 18134 & 6 & 7.0 & 2.16 & 2 & 17 & 9350 & 12 & 6.8 & 0.92 & 2 \\
\hline 15 & 18206 & 14 & 7.9 & 2.17 & 2 & 17 & 9388 & 18 & 6.7 & 0.92 & 2 \\
\hline 15 & 18238 & 8 & 9.0 & 2.18 & 2 & 17 & 9406 & 14 & 7.9 & 0.92 & 2 \\
\hline 16 & 6268 & 4 & 15.4 & 0.68 & 2 & 17 & 9424 & 14 & 8.0 & 0.93 & 2 \\
\hline 16 & 6759 & 5 & 12.8 & 0.71 & 2 & 17 & 9438 & 10 & 6.9 & 0.93 & 2 \\
\hline 16 & 6799 & 5 & 15.1 & 0.72 & 2 & 17 & 9546 & 16 & 6.5 & 0.93 & 2 \\
\hline 16 & 6846 & 8 & 14.6 & 0.72 & 2 & 17 & 9562 & 10 & 5.8 & 0.94 & 2 \\
\hline 16 & 7296 & 6 & 12.8 & 0.75 & 2 & 17 & 9740 & 4 & 4.0 & 0.95 & 2 \\
\hline 16 & 8942 & 6 & 5.0 & 0.88 & 2 & 17 & 10934 & 8 & 3.4 & 1.07 & 2 \\
\hline 16 & 8948 & 6 & 8.7 & 0.88 & 2 & 17 & 11792 & 8 & 6.6 & 1.17 & 2 \\
\hline
\end{tabular}


Table 2B. (continued)

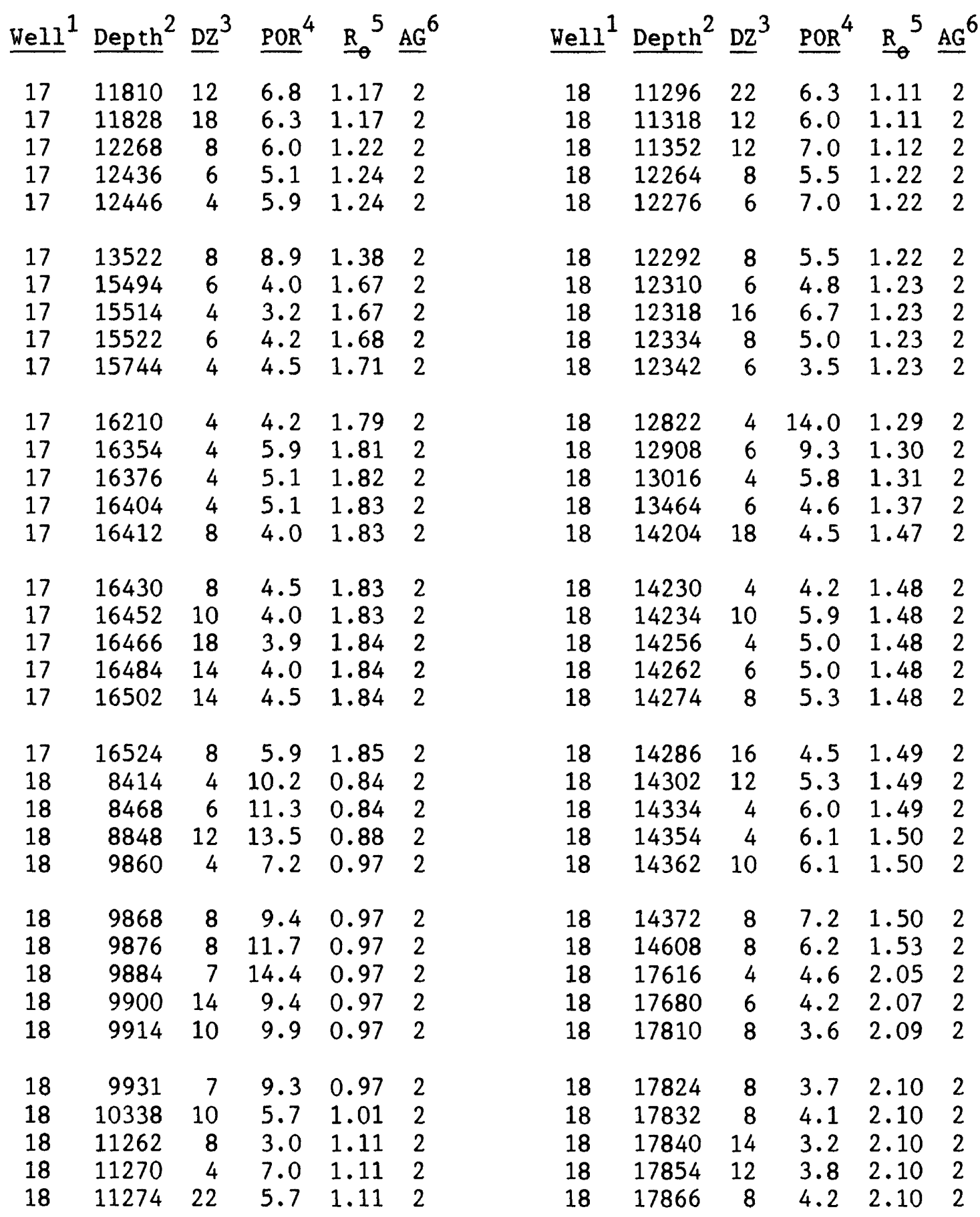


Table 2B. (continued)

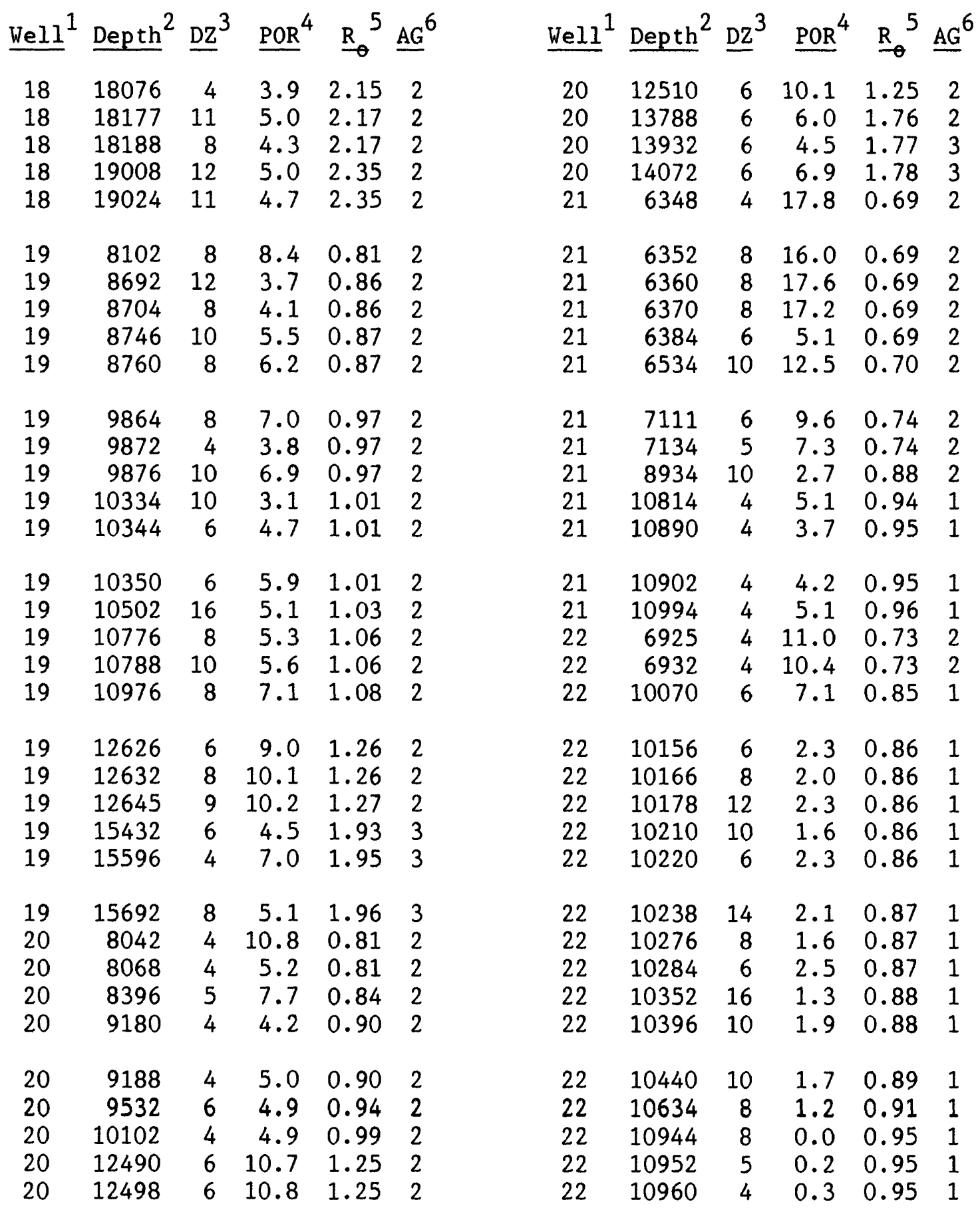


Table 2B. (continued)

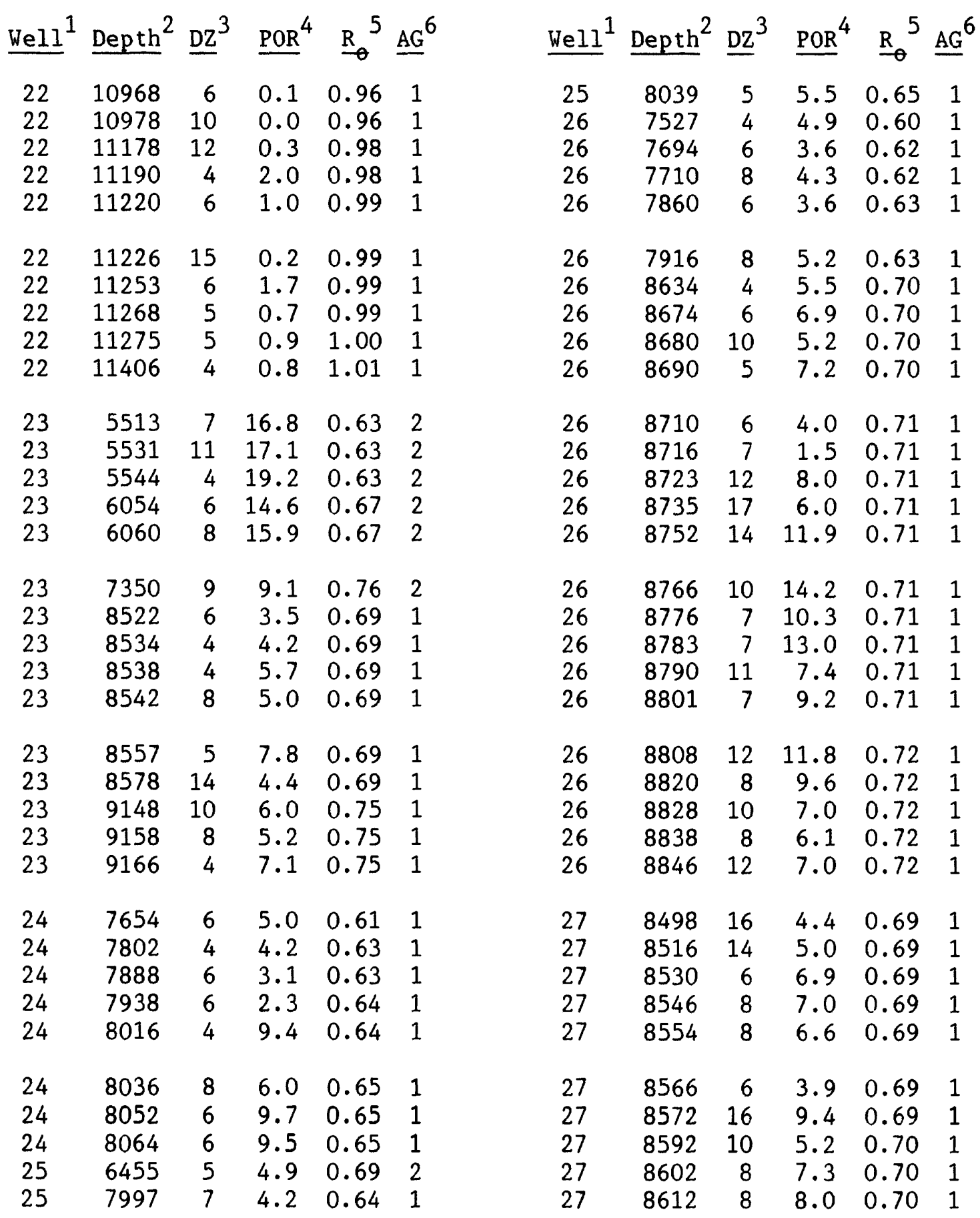


Table 2B. (continued)

\begin{tabular}{|c|c|c|c|c|c|c|c|c|c|c|c|}
\hline Well $^{1}$ & Depth $^{2}$ & $\underline{\mathrm{Dz}}^{3}$ & $\underline{P O R}^{4}$ & $\underline{R}^{5}$ & $\underline{A G}^{6}$ & $\mathrm{Well}^{1}$ & Depth $^{2}$ & $\mathrm{Dz}^{3}$ & $\mathrm{POR}^{4}$ & $\underline{\mathrm{R}}^{5}$ & $\underline{A G}^{6}$ \\
\hline 27 & 8622 & 12 & 7.0 & 0.70 & 1 & 29 & 8834 & 12 & 3.1 & 0.72 & 1 \\
\hline 28 & 9370 & 10 & 5.8 & 0.77 & 1 & 29 & 8848 & 22 & 10.6 & 0.72 & 1 \\
\hline 29 & 5528 & 8 & 19.3 & 0.63 & 2 & 29 & 8870 & 18 & 9.4 & 0.72 & 1 \\
\hline 29 & 5996 & 12 & 16.6 & 0.66 & 2 & 29 & 8888 & 8 & 13.0 & 0.72 & 1 \\
\hline 29 & 6010 & 4 & 15.7 & 0.66 & 2 & 29 & 8896 & 8 & 15.1 & 0.72 & 1 \\
\hline 29 & 6620 & 10 & 3.0 & 0.70 & 2 & 30 & 7058 & 4 & 8.4 & 0.74 & 2 \\
\hline 29 & 6630 & 8 & 5.7 & 0.71 & 2 & 30 & 7966 & 8 & 4.3 & 0.64 & 1 \\
\hline 29 & 6638 & 12 & 3.5 & 0.71 & 2 & 30 & 7974 & 6 & 3.3 & 0.64 & 1 \\
\hline 29 & 6704 & 8 & 2.3 & 0.71 & 2 & 30 & 8020 & 4 & 3.2 & 0.64 & 1 \\
\hline 29 & 6712 & 10 & 4.3 & 0.71 & 2 & 30 & 8116 & 8 & 3.1 & 0.65 & 1 \\
\hline 29 & 7039 & 5 & 7.0 & 0.73 & 2 & 30 & 8838 & 12 & 3.5 & 0.72 & 1 \\
\hline 29 & 7298 & 6 & 2.5 & 0.75 & 2 & 30 & 8860 & 4 & 10.0 & 0.72 & 1 \\
\hline 29 & 7542 & 6 & 4.7 & 0.60 & 1 & 30 & 8864 & 10 & 5.7 & 0.72 & 1 \\
\hline 29 & 7624 & 12 & 5.7 & 0.61 & 1 & 30 & 8874 & 6 & 7.0 & 0.72 & 1 \\
\hline 29 & 7648 & 12 & 3.2 & 0.61 & 1 & 30 & 8884 & 20 & 1.6 & 0.72 & 1 \\
\hline 29 & 7740 & 8 & 3.6 & 0.62 & 1 & 30 & 8912 & 4 & 4.6 & 0.73 & 1 \\
\hline 29 & 7752 & 4 & 4.3 & 0.62 & 1 & 30 & 8916 & 14 & 6.5 & 0.73 & 1 \\
\hline 29 & 7770 & 6 & 3.0 & 0.62 & 1 & 30 & 8946 & 10 & 13.8 & 0.73 & 1 \\
\hline 29 & 7778 & 16 & 3.2 & 0.62 & 1 & 30 & 8962 & 5 & 6.2 & 0.73 & 1 \\
\hline 29 & 7794 & 18 & 4.2 & 0.62 & 1 & 30 & 8967 & 9 & 10.1 & 0.73 & 1 \\
\hline 29 & 7812 & 36 & 3.5 & 0.63 & 1 & 30 & 8976 & 10 & 8.2 & 0.73 & 1 \\
\hline 29 & 7848 & 18 & 4.3 & 0.63 & 1 & 31 & 7666 & 4 & 6.8 & 0.61 & 1 \\
\hline 29 & 7866 & 16 & 4.5 & 0.63 & 1 & 31 & 7744 & 20 & 3.3 & 0.62 & 1 \\
\hline 29 & 7882 & 18 & 3.1 & 0.63 & 1 & 31 & 7764 & 22 & 4.2 & 0.62 & 1 \\
\hline 29 & 7900 & 12 & 2.7 & 0.63 & 1 & 31 & 7786 & 7 & 5.0 & 0.62 & 1 \\
\hline 29 & 7918 & 6 & 2.8 & 0.64 & 1 & 31 & 7793 & 5 & 6.0 & 0.62 & 1 \\
\hline 29 & 7936 & 22 & 3.5 & 0.64 & 1 & 31 & 7894 & 26 & 5.1 & 0.63 & 1 \\
\hline 29 & 7970 & 8 & 2.8 & 0.64 & 1 & 31 & 7928 & 22 & 5.3 & 0.64 & 1 \\
\hline 29 & 7985 & 7 & 1.9 & 0.64 & 1 & 31 & 8048 & 4 & 3.5 & 0.65 & 1 \\
\hline 29 & 8558 & 6 & 3.4 & 0.69 & 1 & 31 & 8165 & 9 & 3.4 & 0.66 & 1 \\
\hline 29 & 8604 & 8 & 1.4 & 0.70 & 1 & 31 & 8176 & 10 & 4.5 & 0.66 & 1 \\
\hline 29 & 8704 & 12 & 2.8 & 0.71 & 1 & 31 & 8968 & 16 & 7.1 & 0.73 & 1 \\
\hline 29 & 8716 & 6 & 5.5 & 0.71 & 1 & 31 & 8988 & 10 & 2.2 & 0.73 & 1 \\
\hline 29 & 8756 & 20 & 5.5 & 0.71 & 1 & 32 & 6326 & 7 & 16.2 & 0.68 & 2 \\
\hline 29 & 8814 & 16 & 7.5 & 0.72 & 1 & 32 & 6352 & 4 & 14.7 & 0.69 & 2 \\
\hline
\end{tabular}


Table 2B. (continued)

$\begin{array}{rrrrrrr}\text { Well }^{1} & \text { Depth }^{2} & \underline{\mathrm{DZ}}^{3} & \underline{\mathrm{POR}}^{4} & \stackrel{\mathrm{R}}{\mathrm{O}}^{5} & \mathrm{AG}^{6} \\ 32 & 6768 & 4 & 10.7 & 0.71 & 2 \\ 32 & 7184 & 4 & 3.5 & 0.58 & 1 \\ 32 & 7188 & 8 & 5.9 & 0.58 & 1 \\ 32 & 7882 & 4 & 5.8 & 0.63 & 1 \\ 32 & 7944 & 4 & 3.5 & 0.64 & 1 \\ 33 & 6193 & 5 & 14.3 & 0.68 & 2 \\ 33 & 10231 & 13 & 3.8 & 0.87 & 1 \\ 33 & 10246 & 8 & 4.0 & 0.87 & 1\end{array}$


TABLE 3A. LIST OF ANADARKO BASIN OIL AND (OR) GAS RESERVOIRS FROM VRICH HYDROCARBON-RESERVOIR POROSITY DATA VERE OBTAINED.

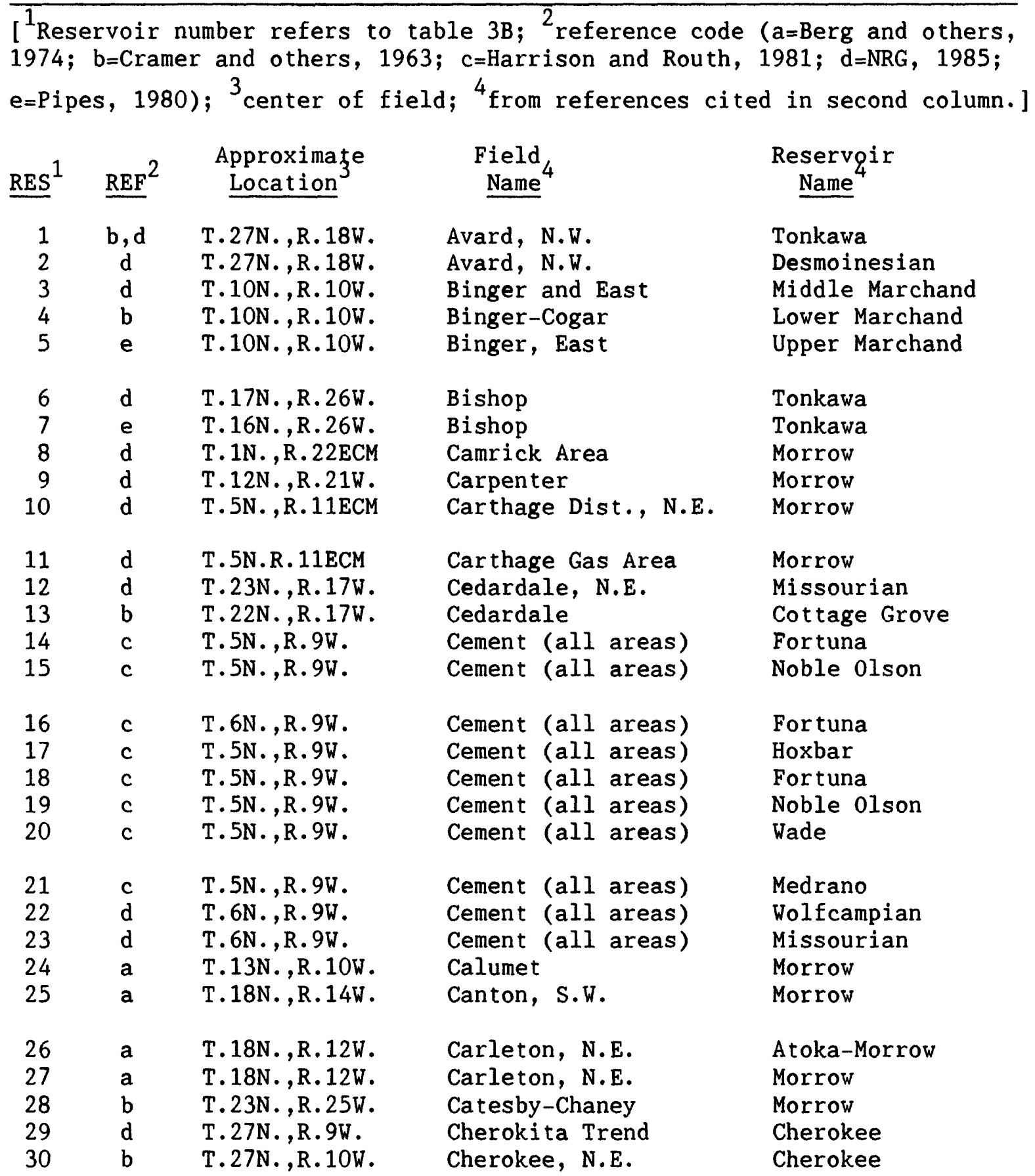


Table 3A. (continued)

\begin{tabular}{|c|c|c|c|c|}
\hline$\underline{\mathrm{RES}}^{1}$ & $\mathrm{REF}^{2}$ & $\begin{array}{l}\text { Approximaţe } \\
\text { Location }\end{array}$ & $\begin{array}{c}\text { Field } \\
\text { Name }\end{array}$ & $\begin{array}{c}\text { Reservqir } \\
\text { Name }\end{array}$ \\
\hline $\begin{array}{l}31 \\
32 \\
33 \\
34 \\
35\end{array}$ & $\begin{array}{c}d \\
b \\
d, e \\
d \\
b\end{array}$ & $\begin{array}{l}\text { T.23N., R. 13W. } \\
\text { T.21N., R.15W. } \\
\text { T.13N., R. 24W. } \\
\text { T.8N., R. 8W. } \\
\text { T.5N.,R. 3W. }\end{array}$ & $\begin{array}{l}\text { Cheyenne Valley } \\
\text { Cheyenne Valley } \\
\text { Cheyenne, West } \\
\text { Chickasha, N.W. } \\
\text { Criner-Payne }\end{array}$ & $\begin{array}{l}\text { Desmoinesian } \\
\text { Red Fork } \\
\text { Upper Morrow } \\
\text { Missourian } \\
\text { Bromide }\end{array}$ \\
\hline $\begin{array}{l}36 \\
37 \\
38 \\
39 \\
40\end{array}$ & $\begin{array}{l}a \\
d \\
d \\
d \\
d\end{array}$ & $\begin{array}{l}\text { T.7N., R. 3W. } \\
\text { T.10N., R. 21W. } \\
\text { T.2N., R. 23ECM } \\
\text { T.4N., R. 10ECM } \\
\text { T.5N., R. 23ECM }\end{array}$ & $\begin{array}{l}\text { Dribble, North } \\
\text { Elk City } \\
\text { Elmwood } \\
\text { Eva, N.W. } \\
\text { Forgan, South }\end{array}$ & $\begin{array}{l}\text { Red Fork } \\
\text { Missourian } \\
\text { Morrow } \\
\text { Cherokee } \\
\text { Morrow }\end{array}$ \\
\hline $\begin{array}{l}41 \\
42 \\
43 \\
44 \\
45\end{array}$ & $\begin{array}{l}d \\
d \\
a \\
b \\
d\end{array}$ & $\begin{array}{l}\text { T.21N., R. 24W. } \\
\text { T.20N., R. 24W. } \\
\text { T.13N., R.10W. } \\
\text { T.8N., R. 17W. } \\
\text { T.6N., R. 21ECM }\end{array}$ & $\begin{array}{l}\text { Gage, South } \\
\text { Gage, South } \\
\text { Geary } \\
\text { Gotebo Area, North } \\
\text { Greenough, West }\end{array}$ & $\begin{array}{l}\text { Morrow } \\
\text { Morrow } \\
\text { Morrow } \\
\text { Springer } \\
\text { Desmoinesian }\end{array}$ \\
\hline $\begin{array}{l}46 \\
47 \\
48 \\
49 \\
50\end{array}$ & $\begin{array}{l}d \\
d \\
a \\
d \\
e\end{array}$ & $\begin{array}{l}\text { T. 3N. , R. 17ECM } \\
\text { T.18N., R. 26W. } \\
\text { T.17N., R. 11W. } \\
\text { T. 24N., R. } 4 \mathrm{~W} . \\
\text { T. } 24 \text { N. , R. } 4 \mathrm{~W} .\end{array}$ & $\begin{array}{l}\text { Hardesty, North } \\
\text { Higgins, South } \\
\text { Hitchcock } \\
\text { Hunter, South } \\
\text { Hunter, South }\end{array}$ & $\begin{array}{l}\text { Morrow } \\
\text { Morrow } \\
\text { Atoka } \\
\text { Layton } \\
\text { Misener }\end{array}$ \\
\hline $\begin{array}{l}51 \\
52 \\
53 \\
54 \\
55\end{array}$ & $\begin{array}{l}d \\
b \\
b \\
b \\
b\end{array}$ & $\begin{array}{l}\text { T. 5N. , R. 9ECM } \\
\text { T. 5N., R. 9ECM } \\
\text { T.26N., R. } 25 \mathrm{~W} . \\
\text { T.26N., R. } 25 \mathrm{~W} . \\
\text { T.26N., R. } 25 \mathrm{~W} .\end{array}$ & $\begin{array}{l}\text { Keys Area } \\
\text { Keys } \\
\text { Laverne } \\
\text { Laverne } \\
\text { Laverne }\end{array}$ & $\begin{array}{l}\text { Morrow } \\
\text { Keys } \\
\text { Hoover } \\
\text { Tonkawa } \\
\text { Morrow }\end{array}$ \\
\hline $\begin{array}{l}56 \\
57 \\
58 \\
59 \\
60\end{array}$ & $\begin{array}{l}b \\
b \\
b \\
d \\
d\end{array}$ & $\begin{array}{l}\text { T.18N., R. 18W. } \\
\text { T.5N., R. 21ECM } \\
\text { T.5N.,R.21ECM } \\
\text { T.1N., R. 26ECM } \\
\text { T.1N., R. 26ECM }\end{array}$ & $\begin{array}{l}\text { Lenora } \\
\text { Light Gas Area } \\
\text { Light Gas Area } \\
\text { Logan, South } \\
\text { Logan, South }\end{array}$ & $\begin{array}{l}\text { Morrow } \\
\text { Upper Morrow } \\
\text { Basal Morrow } \\
\text { Morrow } \\
\text { Tonkawa }\end{array}$ \\
\hline $\begin{array}{l}61 \\
62 \\
63 \\
64 \\
65\end{array}$ & $\begin{array}{l}d \\
d \\
b \\
b \\
b, d\end{array}$ & $\begin{array}{l}\text { T. 28N., R. 21W. } \\
\text { T.28N., R. 21W. } \\
\text { T.24N., R. 24W. } \\
\text { T. 24N., R. 24W. } \\
\text { T.28N., R. 3W. }\end{array}$ & $\begin{array}{l}\text { Lovedale } \\
\text { Lovedale } \\
\text { Luther Hill } \\
\text { Luther Hill } \\
\text { Mayflower, N.W. }\end{array}$ & $\begin{array}{l}\text { Morrow } \\
\text { Tonkawa } \\
\text { Lower Tonkawa } \\
\text { Lower Morrow } \\
\text { Red Fork }\end{array}$ \\
\hline
\end{tabular}


Table 3A. (continued)

\begin{tabular}{|c|c|c|c|c|}
\hline $\mathrm{RES}^{1}$ & $\mathrm{REF}^{2}$ & $\begin{array}{l}\text { Approximaţe } \\
\text { Location }\end{array}$ & $\begin{array}{l}\text { Field } \\
\text { Name }\end{array}$ & $\begin{array}{c}\text { Reservoir } \\
\text { Name }\end{array}$ \\
\hline $\begin{array}{l}66 \\
67 \\
68 \\
69 \\
70\end{array}$ & $\begin{array}{l}e \\
b \\
d \\
a \\
a\end{array}$ & $\begin{array}{l}\text { T.8N., R. 7W. } \\
\text { T.27N.,R.24W. } \\
\text { T.5N.,R.15ECM } \\
\text { T.7N.,R.8W. } \\
\text { T.24N.,R.13W. }\end{array}$ & $\begin{array}{l}\text { Minco, S.W. } \\
\text { Mocane-Laverne } \\
\text { Mouser } \\
\text { Norge and Verden, N.W. } \\
\text { Oakdale, N.W. }\end{array}$ & $\begin{array}{l}\text { Springer } \\
\text { Morrow } \\
\text { Morrow } \\
\text { Marchand } \\
\text { Red Fork }\end{array}$ \\
\hline $\begin{array}{l}71 \\
72 \\
73 \\
74 \\
75\end{array}$ & $\begin{array}{l}d \\
a \\
b \\
d \\
b\end{array}$ & $\begin{array}{l}\text { T.17N., R. 14W. } \\
\text { T.18N.,R.14W. } \\
\text { T.15N., R. } 7 \mathrm{~W} . \\
\text { T.20N., R. } 11 \mathrm{~W} . \\
\text { T.19N., R. } 11 \mathrm{~W} .\end{array}$ & $\begin{array}{l}\text { Oakwood, North } \\
\text { Oakwood, N.W. } \\
\text { Okarche, North } \\
\text { Okeene, N.W. } \\
\text { Okeene, N.W. }\end{array}$ & $\begin{array}{l}\text { Morrow } \\
\text { Morrow } \\
\text { Manning } \\
\text { Red Fork } \\
\text { Red Fork }\end{array}$ \\
\hline $\begin{array}{l}76 \\
77 \\
78 \\
79 \\
80\end{array}$ & $\begin{array}{l}c \\
c \\
d \\
d \\
c\end{array}$ & $\begin{array}{l}\text { T.11N., R. 2W. } \\
\text { T.11N.,R.2W. } \\
\text { T. } 5 \mathrm{~N} ., \text { R. } 13 \mathrm{ECM} \\
\text { T.5N.,R.13ECM } \\
\text { T.4N.,R.13ECM }\end{array}$ & $\begin{array}{l}\text { Oklahoma City } \\
\text { Oklahoma City } \\
\text { Postle } \\
\text { Postle } \\
\text { Postle-Hough }\end{array}$ & $\begin{array}{l}\text { Prue } \\
\text { Wilcox } \\
\text { Morrow } \\
\text { Cherokee } \\
\text { Upper Cherokee }\end{array}$ \\
\hline $\begin{array}{l}81 \\
82 \\
83 \\
84 \\
85\end{array}$ & $\begin{array}{l}\mathrm{c} \\
\mathrm{c} \\
\mathrm{c} \\
\mathrm{d} \\
\mathrm{a}\end{array}$ & $\begin{array}{l}\text { T. 5N., R. 13ECM } \\
\text { T.4N.,R.14ECM } \\
\text { T.4N., R. 14ECM } \\
\text { T.16N., R. 16W. } \\
\text { T.13N.,R. } 26 \mathrm{~W} .\end{array}$ & $\begin{array}{l}\text { Postle-Hough } \\
\text { Postle-Hough } \\
\text { Postle-Hough } \\
\text { Putnam } \\
\text { Reydon, W. and N.W. }\end{array}$ & $\begin{array}{l}\text { Upper Morrow } \\
\text { Upper Morrow } \\
\text { Morrow } \\
\text { Desmoinesian } \\
\text { Upper Morrow }\end{array}$ \\
\hline $\begin{array}{l}86 \\
87 \\
88 \\
89 \\
90\end{array}$ & $\begin{array}{l}d \\
d \\
b \\
d \\
d\end{array}$ & $\begin{array}{l}\text { T. 25N., R. 4W. } \\
\text { T.25N.,R. } 4 \mathrm{~W} . \\
\text { T. } 25 \mathrm{~N} ., \text { R. } 4 \mathrm{~W} . \\
\text { T. } 5 \mathrm{~N} ., \text { R. } 12 \mathrm{ECM} \\
\text { T.25N., R. } 3 \mathrm{~W} .\end{array}$ & $\begin{array}{l}\text { Rich Valley } \\
\text { Rich Valley } \\
\text { Rich Valley Area } \\
\text { Richland, Central, N. } \\
\text { Saltfork, S.E. }\end{array}$ & $\begin{array}{l}\text { Mississippian } \\
\text { Simpson } \\
\text { Wilcox } \\
\text { Morrow } \\
\text { Skinner }\end{array}$ \\
\hline $\begin{array}{l}91 \\
92 \\
93 \\
94 \\
95\end{array}$ & $\begin{array}{l}b \\
d \\
d \\
a \\
d\end{array}$ & $\begin{array}{l}\text { T.20N., R. 16W. } \\
\text { T.8N.,R.20W. } \\
\text { T.21N., R. 21W. } \\
\text { T.21N., R. 21W. } \\
\text { T.20N.,R.8W. }\end{array}$ & $\begin{array}{l}\text { Seiling, N.E. } \\
\text { Sentinel, West } \\
\text { Sharon, West } \\
\text { Sharon, West } \\
\text { Sooner Trend }\end{array}$ & $\begin{array}{l}\text { Cottage Grove } \\
\text { Granite wash } \\
\text { Morrow } \\
\text { Sharon } \\
\text { Desmoinesian }\end{array}$ \\
\hline $\begin{array}{r}96 \\
97 \\
98 \\
99 \\
100\end{array}$ & $\begin{array}{l}d \\
d \\
d \\
a \\
d\end{array}$ & $\begin{array}{l}\text { T. 5N., R. 10ECM } \\
\text { T. } 23 \mathrm{~N} ., \text { R. } 22 \mathrm{~W} . \\
\text { T. } 28 \mathrm{~N} ., \text { R. } 8 \mathrm{~W} . \\
\text { T. } 8 \mathrm{~N} ., \text { R. } 4 \mathrm{~W} . \\
\text { T. } 14 \mathrm{~N},, \mathrm{R} .10 \mathrm{~W} .\end{array}$ & $\begin{array}{l}\text { Sturgis, East } \\
\text { Tangier } \\
\text { Wakita Trend } \\
\text { Washington, E. } \\
\text { Watonga-Chickasha }\end{array}$ & $\begin{array}{l}\text { Morrow } \\
\text { Morrow } \\
\text { Cherokee } \\
\text { Osborne } \\
\text { Morrow }\end{array}$ \\
\hline
\end{tabular}


Table 3A. (continued)

\begin{tabular}{|c|c|c|c|c|}
\hline $\operatorname{RES}^{1}$ & $\mathrm{REF}^{2}$ & $\begin{array}{l}\text { Approximaţe } \\
\text { Location }\end{array}$ & $\begin{array}{l}\text { Field } \\
\text { Name }\end{array}$ & $\begin{array}{c}\text { Reservgir } \\
\text { Name }\end{array}$ \\
\hline $\begin{array}{l}101 \\
102 \\
103 \\
104 \\
105\end{array}$ & $\begin{array}{c}d \\
d \\
b, d \\
d \\
d\end{array}$ & $\begin{array}{l}\text { T.14N., R. 10W. } \\
\text { T.14N., R. 10W. } \\
\text { T.25N., R. 16W. } \\
\text { T.22N., R.19W. } \\
\text { T.29N., R.17W. }\end{array}$ & $\begin{array}{l}\text { Watonga-Chickasha } \\
\text { Wa tonga-Chickasha } \\
\text { Waynoka, N.E. } \\
\text { Woodward, S.E. } \\
\text { Yellows tone }\end{array}$ & $\begin{array}{l}\text { Springer } \\
\text { Atoka } \\
\text { Cottage Grove } \\
\text { Morrow } \\
\text { Simpson }\end{array}$ \\
\hline
\end{tabular}


TABLE 3B. ANADARKO BASIN HYDROCARBON-RESERVOIR POROSITY DATA

$\left[{ }^{1}\right.$ Reservoir number from table $3 \mathrm{~A}$; 2 average reservoir porosity cited in references $(\%) ;{ }^{3} \mathrm{ft} ;{ }^{4}$ age code (1=Lower Paleozoic, 2=Pennsylvanian, $3=$ Permian); ${ }^{5}$ equivalent vitrinite reflectance (\%).]

\begin{tabular}{|c|c|c|c|c|c|c|c|c|c|}
\hline$\underline{R E S}^{1}$ & $\underline{\mathrm{POR}}^{2}$ & $\underline{D e p t h}^{3}$ & & $4 \underline{R}^{5}$ & $\underline{\mathrm{RES}}^{1}$ & $\underline{\mathrm{POR}}^{2}$ & Depth $^{3}$ & $\underline{\mathrm{AG}}$ & ${ }^{4} \underline{R}^{5}$ \\
\hline $\begin{array}{l}1 \\
2 \\
3 \\
4 \\
5\end{array}$ & $\begin{array}{r}14.0 \\
12.0 \\
8.5 \\
9.5 \\
8.5\end{array}$ & $\begin{array}{l}4800 \\
5361 \\
9817 \\
9840 \\
9930\end{array}$ & $\begin{array}{l}2 \\
2 \\
2 \\
2 \\
2\end{array}$ & $\begin{array}{l}0.59 \\
0.62 \\
0.96 \\
0.96 \\
0.97\end{array}$ & $\begin{array}{l}36 \\
37 \\
38 \\
39 \\
40\end{array}$ & $\begin{array}{l}11.0 \\
16.8 \\
16.0 \\
14.0 \\
13.0\end{array}$ & $\begin{array}{l}9608 \\
8800 \\
7651 \\
4022 \\
6430\end{array}$ & $\begin{array}{l}2 \\
2 \\
2 \\
2 \\
2\end{array}$ & $\begin{array}{l}0.94 \\
0.87 \\
0.78 \\
0.55 \\
0.69\end{array}$ \\
\hline $\begin{array}{r}6 \\
7 \\
8 \\
9 \\
10\end{array}$ & $\begin{array}{r}12.0 \\
12.0 \\
12.7 \\
9.0 \\
14.0\end{array}$ & $\begin{array}{r}7700 \\
7950 \\
6376 \\
17002 \\
4456\end{array}$ & $\begin{array}{l}2 \\
2 \\
2 \\
2 \\
2\end{array}$ & $\begin{array}{l}0.78 \\
0.80 \\
0.69 \\
1.93 \\
0.57\end{array}$ & $\begin{array}{l}41 \\
42 \\
43 \\
44 \\
45\end{array}$ & $\begin{array}{l}10.7 \\
12.0 \\
11.0 \\
13.5 \\
10.0\end{array}$ & $\begin{array}{r}5080 \\
9640 \\
10420 \\
5670 \\
4636\end{array}$ & $\begin{array}{l}2 \\
2 \\
2 \\
2 \\
2\end{array}$ & $\begin{array}{l}0.61 \\
0.95 \\
1.02 \\
0.64 \\
0.58\end{array}$ \\
\hline $\begin{array}{l}11 \\
12 \\
13 \\
14 \\
15\end{array}$ & $\begin{array}{l}15.0 \\
13.0 \\
15.0 \\
19.2 \\
19.2\end{array}$ & $\begin{array}{l}4496 \\
6133 \\
6360 \\
2000 \\
2035\end{array}$ & $\begin{array}{l}2 \\
2 \\
2 \\
3 \\
3\end{array}$ & $\begin{array}{l}0.57 \\
0.67 \\
0.69 \\
0.45 \\
0.45\end{array}$ & $\begin{array}{l}46 \\
47 \\
48 \\
49 \\
50\end{array}$ & $\begin{array}{r}16.0 \\
7.0 \\
15.0 \\
10.7 \\
10.7\end{array}$ & $\begin{array}{r}6269 \\
11920 \\
8082 \\
5455 \\
6150\end{array}$ & $\begin{array}{l}2 \\
2 \\
2 \\
2 \\
1\end{array}$ & $\begin{array}{l}0.68 \\
1.18 \\
0.81 \\
0.63 \\
0.50\end{array}$ \\
\hline $\begin{array}{l}16 \\
17 \\
18 \\
19 \\
20\end{array}$ & $\begin{array}{l}22.0 \\
17.1 \\
10.0 \\
20.2 \\
10.0\end{array}$ & $\begin{array}{l}2200 \\
2400 \\
2450 \\
3400 \\
3850\end{array}$ & $\begin{array}{l}3 \\
2 \\
3 \\
3 \\
2\end{array}$ & $\begin{array}{l}0.46 \\
0.47 \\
0.47 \\
0.52 \\
0.54\end{array}$ & $\begin{array}{l}51 \\
52 \\
53 \\
54 \\
55\end{array}$ & $\begin{array}{l}16.0 \\
16.0 \\
18.0 \\
18.0 \\
14.0\end{array}$ & $\begin{array}{l}4253 \\
4700 \\
4285 \\
5500 \\
7200\end{array}$ & $\begin{array}{l}2 \\
2 \\
2 \\
2 \\
2\end{array}$ & $\begin{array}{l}0.56 \\
0.58 \\
0.56 \\
0.63 \\
0.75\end{array}$ \\
\hline $\begin{array}{l}21 \\
22 \\
23 \\
24 \\
25\end{array}$ & $\begin{array}{l}16.5 \\
18.1 \\
14.5 \\
11.5 \\
10.0\end{array}$ & $\begin{array}{l}5826 \\
1900 \\
7056 \\
8769 \\
8850\end{array}$ & $\begin{array}{l}2 \\
3 \\
2 \\
2 \\
2\end{array}$ & $\begin{array}{l}0.65 \\
0.45 \\
0.74 \\
0.87 \\
0.88\end{array}$ & $\begin{array}{l}56 \\
57 \\
58 \\
59 \\
60\end{array}$ & $\begin{array}{l}10.0 \\
15.0 \\
13.0 \\
14.5 \\
14.0\end{array}$ & $\begin{array}{r}10390 \\
6150 \\
6450 \\
8256 \\
5967\end{array}$ & $\begin{array}{l}2 \\
2 \\
2 \\
2 \\
2\end{array}$ & $\begin{array}{l}1.02 \\
0.67 \\
0.69 \\
0.83 \\
0.66\end{array}$ \\
\hline $\begin{array}{l}26 \\
27 \\
28 \\
29 \\
30\end{array}$ & $\begin{array}{l}15.0 \\
10.0 \\
14.0 \\
17.0 \\
17.0\end{array}$ & $\begin{array}{l}8335 \\
8475 \\
8150 \\
4880 \\
5170\end{array}$ & $\begin{array}{l}2 \\
2 \\
2 \\
2 \\
2\end{array}$ & $\begin{array}{l}0.83 \\
0.84 \\
0.82 \\
0.59 \\
0.61\end{array}$ & $\begin{array}{l}61 \\
62 \\
63 \\
64 \\
65\end{array}$ & $\begin{array}{l}11.0 \\
10.0 \\
17.0 \\
14.0 \\
20.0\end{array}$ & $\begin{array}{l}6088 \\
5009 \\
5850 \\
7700 \\
4534\end{array}$ & $\begin{array}{l}2 \\
2 \\
2 \\
2 \\
2\end{array}$ & $\begin{array}{l}0.67 \\
0.60 \\
0.65 \\
0.78 \\
0.58\end{array}$ \\
\hline $\begin{array}{l}31 \\
32 \\
33 \\
34 \\
35\end{array}$ & $\begin{array}{l}12.0 \\
12.0 \\
14.7 \\
10.0 \\
15.0\end{array}$ & $\begin{array}{r}6307 \\
7100 \\
15090 \\
8884 \\
9715\end{array}$ & $\begin{array}{l}2 \\
2 \\
2 \\
2 \\
1\end{array}$ & $\begin{array}{l}0.68 \\
0.74 \\
1.61 \\
0.88 \\
0.81\end{array}$ & $\begin{array}{l}66 \\
67 \\
68 \\
69 \\
70\end{array}$ & $\begin{array}{l}18.0 \\
12.0 \\
17.5 \\
10.0 \\
10.5\end{array}$ & $\begin{array}{r}12470 \\
6260 \\
6380 \\
10270 \\
6460\end{array}$ & $\begin{array}{l}2 \\
2 \\
2 \\
2 \\
2\end{array}$ & $\begin{array}{l}1.24 \\
0.68 \\
0.69 \\
1.00 \\
0.69\end{array}$ \\
\hline
\end{tabular}


Table 3B. (continued)

\begin{tabular}{|c|c|c|c|c|}
\hline $\mathrm{RES}^{1}$ & $\mathrm{POR}^{2}$ & Depth $^{3}$ & $\underline{A G}^{4}$ & ${ }^{4} \mathrm{R}^{5}$ \\
\hline 71 & 13.0 & 9066 & 2 & 0.89 \\
\hline 72 & 13.0 & 9425 & 2 & 0.93 \\
\hline 73 & 9.5 & 7900 & 1 & 0.63 \\
\hline 74 & 11.0 & 7132 & 2 & 0.74 \\
\hline 75 & 11.0 & 7200 & 2 & 0.75 \\
\hline 76 & 13.6 & 6360 & 2 & 0.69 \\
\hline 77 & 18.0 & 5750 & 1 & 0.48 \\
\hline 78 & 15.7 & 6039 & 2 & 0.67 \\
\hline 79 & 20.0 & 5423 & 2 & 0.63 \\
\hline 80 & 20.0 & 5485 & 2 & 0.63 \\
\hline 81 & 15.4 & 6100 & 2 & 0.67 \\
\hline 82 & 16.0 & 6150 & 2 & 0.67 \\
\hline 83 & 15.7 & 6500 & 2 & 0.70 \\
\hline 84 & 7.5 & 8652 & 2 & 0.86 \\
\hline 85 & 13.0 & 14900 & 2 & 1.58 \\
\hline 86 & 16.9 & 5176 & 1 & 0.44 \\
\hline 87 & 11.5 & 5816 & 1 & 0.48 \\
\hline 88 & 11.5 & 5832 & 1 & 0.48 \\
\hline 89 & 17.0 & 5526 & 2 & 0.63 \\
\hline 90 & 14.4 & 4942 & 2 & 0.60 \\
\hline 91 & 13.5 & 6940 & 2 & 0.73 \\
\hline 92 & 17.0 & 5600 & 2 & 0.64 \\
\hline 93 & 14.0 & 9038 & 2 & 0.89 \\
\hline 94 & 13.0 & 9125 & 2 & 0.90 \\
\hline 95 & 13.0 & 5620 & 2 & 0.64 \\
\hline 96 & 16.0 & 4267 & 2 & 0.56 \\
\hline 97 & 8.0 & 8337 & 2 & 0.83 \\
\hline 98 & 13.5 & 4700 & 2 & 0.58 \\
\hline 99 & 12.5 & 8500 & 2 & 0.85 \\
\hline 100 & 10.5 & 8179 & 2 & 0.82 \\
\hline 101 & 18.0 & 11192 & 2 & 1.10 \\
\hline 102 & 15.0 & 9668 & 2 & 0.95 \\
\hline 103 & 15.0 & 5472 & 2 & 0.63 \\
\hline 104 & 13.6 & 8233 & 2 & 0.82 \\
\hline 105 & 13.7 & 5936 & 1 & 0.49 \\
\hline
\end{tabular}




\section{REFERENCES}

\section{General}

Cleveland, W.S., 1985, The elements of graphing data: Monterey, California, Wadsworth Advanced Books and Software, $323 \mathrm{p}$.

Schmoker, J.W., 1986, 0il generation in the Anadarko basin, Oklahoma and Texas: Modeling using Lopatin's method: Oklahoma Geological Survey Special Publication 86-3, 40 p.

\section{Box-Diagram Porosity Data Set (Tables 1A and 1B)}

Amato, R.V., and Bebout, J.W., eds., 1978, Geological and operational summary, COST No. GE-1 well, southeast Georgia Embayment area, South Atlantic OCS: U.S. Geological Survey Open-File Report 78-668, 122 p.

Anders, D.E., 1990, Thermal maturation in the Unita basin, Utah (abs.), in Carter, L.M.H., ed., USGS research on energy resources--1990 program and abstracts, sixth V.E. McKelvey forum on mineral and energy resources: U.S. Geological Survey Circular 1060, p. 2-3.

Barker, C.E., 1979, Vitrinite reflectance geothermometry in the Cerro Prieto geothermal system, Baja California, Mexico: Master's thesis, University of California at Riverside, Riverside, California, $126 \mathrm{p}$.

Bostick, N.H., 1979, Microscopic measurement of the level of catagenesis of solid organic matter in sedimentary rocks to aid exploration for petroleum and to determine former burial temperatures--a review, in Scholle, P.A., and Schluger, P.R., eds., Aspects of diagenesis: Society of Economic Paleontologists and Mineralogists Special Publication No. 26, p. 17-43.

Bostick, N.H., and Freeman, V.L., 1984, Tests of vitrinite reflectance and paleotemperature models at the Multiwell Experiment Site, Piceance Creek basin, Colorado, in Spencer, C.W., and Keighin, C.W., eds., Geologic studies in support of the U.S. Department of Energy Multiwell Experiment, Garfield County, Colorado: U.S. Geological Survey Open-File Report 84-757, p. 110-120.

Brown, D.M., McAlpine, K.D., and Yole, R.W., 1989, Sedimentology and sandstone diagenesis of Hibernia Formation in Hibernia oil field, Grand Banks of Newforndland: AAPG Bulletin, v. 73, no. 5, p. 557-575.

Dixon, S.A., Summers, D.M., and Surdam, R.C., 1989, Diagenesis and preservation of porosity in Norphlet Formation (Upper Jurassic), southern Alabama: AAPG Bulletin, v. 73, no. 6, p.707-728. 
Hansley, P.L., and Johnson, R.C., 1980, Mineralogy and diagenesis of low-permeability sandstones of Late Cretaceous age, Piceance Creek basin, northwestern Colorado: The Mountain Geologist, v.17, no. 4, p. 88-106.

Harris, N.B., 1989, Reservoir geology of Fangst Group (Middle Jurassic), Heidron field offshore mid-Norway: AAPG Bulletin, v. 73 , no. 11, p. 81-96.

Helmold, K.P., and van de Kamp, P.C., 1984, Diagenetic mineralogy and controls on albitization and laumontite formation in Paleogene arkoses, Santa Ynez Mountains, California, in McDonald, D.A., and Surdam, R.C., eds., Clastic diagenesis: AAPG Memoir 37, p. 239-276.

Honda, Hiromi, 1981, Diagenesis and reservoir quality of the Norphlet sandstone (Upper Jurassic), Hatters Pond area, Mobile County, Alabama: Master's thesis, University of Texas at Austin, Austin, Texas, 213 p.

Houseknecht, D.S., 1988, Intergranular pressure solution in four quartzose sandstones: Journal of Sedimentary Petrology, v. 58, p. 228-246.

Issler, D.R., 1984, Calculation of organic maturation levels for offshore eastern Canada--implications for general application of Lopatin's method: Canadian Journal of Earth Science, v. 21, p. 477-488.

James, S.W., 1989, Diagenetic history and reservoir characteristics of a deep Minnelusa reservoir, Hawk Point field, Powder River basin, Wyoming, in Coalson, E.B., ed., Petrogenesis and petrophysics of selected sandstone reservoirs of the Rocky Mountain region: Rocky Mountain Association of Geologists, Denver, p. 81-96.

Johnson, R.C., and Nuccio, V.F., 1986, Structural and thermal history of the Piceance Creek basin, western Colorado, in relation to hydrocarbon occurrence in the Mesaverde Group, in Spencer, C.W., and Mast, R.F., eds., Geology of tight gas reservoirs: AAPG Studies in Geology \#24, p. 165-205.

Laughrey, C.S., and Harper, J.A., 1986, Comparisons of Upper Devonian and Lower Silurian tight formations in Pennsylvania--geological and engineering characteristics, in Spencer, C.W., and Mast, R.F., eds., Geology of tight gas reservoirs: AAPG Studies in Geology \#24, p. 9-43.

Lyons, D.J., and van de Kamp, P.C., 1980, Subsurface geological and geophysical study of the Cerro Prieto geothermal field, Baja California, Mexico: Lawrence Berkeley Laboratory LBL-10540, 95 p.

Magoon, L.B., ed., 1986, Geologic studies of the Lower Cook Inlet Cost No. 1 well, Alaska outer continental shelf: U.S. Geological Survey Bulletin 1596, 99 p. 
McBride, E.F., Land, L.S., and Mack, L.E., 1987, Diagensis of eolian and fluvial feldspathic sandstones, Norphlet Formation (Upper Jurassic), Rankin County, Mississippi, and Mobile County, Alabama: AAPG Bulletin, v. 71, no. 9, p. 1019-1034.

Mudford, B.S., and Best, M.E., 1989, Venture gas field, offshore Nova Scotia: Case study of overpressuring in region of low sedimentation rate: AAPG Bulletin, V. 73, no. 11, p. 1383-1396.

Pitman, J.K., Fouch, T.D., and Goldhaber, M.B., 1982, Depositional setting and diagenetic evolution of some Tertiary unconventional reservoir rocks, Unita basin, Utah: AAPG Bulletin, v. 66, no. 10, p. 1581-1596.

Pitman, J.K., Franczyk, K.J., and Anders, K.E., 1988, Diagenesis and burial history of nonmarine Upper Cretaceous rocks in the central Uinta basin, Utah: U.S. Geological Survey Bulletin 1787-D, 24 p.

Schmidt, V., and McDonald, D.A,. 1979, The role of secondary porosity in the course of sandstone diagenesis, in Scholle, P.A., and Schluger, P.R., eds., Aspects of diagenesis: Society of Economic Paleontologists and Mineralogists Special Publication No. 26, p. 175-207.

Scholle, P.A., ed., 1977, Geological studies on the COST No. B-2 well, U.S. mid-Atlantic outer continental shelf area: U.S. Geological Survey Circular $750,71 \mathrm{p}$.

Scholle, P.A., ed., 1979, Geological studies of the COST GE-1 well, United States South Atlantic outer continental shelf area: U.S. Geological Survey Circular 800, 114 p.

Streib, D.L., 1981, Distribution of gas, organic carbon, and vitrinite reflectance in the eastern Devonian gas shales and their relationship to the geologic framework: U.S. Department of Energy Morgantown Energy Technology Center DOE/MC/08216-1276, 262 p.

Taylor, T.R., 1990, The influence of calcite dissolution on reservoir porosity in Miocene sandstones, Picaroon field, offshore Texas Gulf Coast: Journal of Sedimentary Petrology, v. 60, no. 3, p. 322-334.

Turner, R.F., ed., 1983a, Geological and operational summary, Norton Sound COST No. 1 well, Norton Sound, Alaska: U.S. Geological Survey Open-File Report 83-124, 164 p.

Turner, R.F., ed., 1983b, Geological and operational summary, Norton Sound COST No. 2 well, Norton Sound, Alaska: U.S. Geological Survey Open-File Report 83-557, 154 p.

Turner, R.F., ed., 1984a, Geological and operational summary, Naverin basin COST No. 1 well, Bering Sea, Alaska: U.S. Minerals Management Service OCS Report MMS 84-0031, 245 p. 
Turner, R.F., ed., 1984b, Geological and operational summary, St. George basin COST No. 1 well, Bering Sea, Alaska: U.S. Minerals Management Service OCS Report MMS 84-0016, 105 p.

Turner, R.F., ed., 1984c, Geological and operational summary, St. George basin COST No. 2 well, Bering Sea, Alaska: U.S. Minerals Management Service OCS Report MMS 84-0018, 100 p.

Turner, R.F., ed., 1987, Geological and operational summary, Kodiak Shelf Stratigraphic Test Wells, western Gulf of Alaska: U.S. Minerals Management Service 0CS Report MMS 87-0109, 341 p.

Turner, R.F., ed., 1988, Geological and operational summary, North Aleutian Shelf COST No. 1 well, Bering Sea, Alaska: U.S. Minerals Management Service OCS Report MMS 88-0089, 256 p.

van de Kamp, P.C., 1988, Stratigraphy and diagenetic alteration of Ellesmerian sequence siliciclastic rocks, North Slope, Alaska, in Gryc, George, ed., Geology and exploration of the National Petroleum Reserve in Alaska, 1974 to 1982: U.S. Geological Survey Professional Paper 1399, p. 833-854.

Wendlandt, R.F., and Bhuyan, Kamal, 1990, Estimation of mineralogy and lithology from geochemical log measurements: AAPG Bulletin, v. 74, no. 6, p. 837-856.

Wills, J.C., Bolm, J.G., Stewart, G.H., Turner, R.F., Lynch, M.B., Petering, G.W., Parker, J., and Schoof, B., 1978, Geological and operational summary, Atlantic Richfield Lower Cook Inlet, Alaska, COST well No. 1: U.S. Geological Survey Open-File Report 78-145, 46 p.

\section{Anadarko Basin Hydrocarbon-Reservoir Porosity Data Set (Tables 3A and 3B)}

Berg, 0.R., Koinm, D.N., and Richardson, D.E., eds., 1974, $0 i 1$ and gas fields of Oklahoma: Oklahoma City Geological Society Reference Report Supplement I, $54 \mathrm{p}$.

Cramer, R.D., Gatlin, Leroy, and Wessman H.G., eds., 1963, 0il and gas fields of Oklanoma: Oklahoma City Geological Society Reference Report Volume I, 200 p.

Harrison, W.E., and Routh, D.L., compilers, 1981, Reservoir and fluid characteristics of selected oil fields in 0klahoma: Oklahoma Geological Survey Special Publication 81-1, 317 p. 
NRG Associates, Inc., 1985, Significant oil and gas fields of the United States: The field/reservoir clusters of the United States: Available from Nehring Associates, Inc., P.0. Box 1655, Colorado Springs, C0 80901 .

Pipes, P.B., ed., 1980, 0il and gas fields of Oklahoma: Oklahoma City Geological Society Reference Report Supplement II, 30 p.

\section{Interpretive Papers Based on the Data of this Report}

Hester, T.C., 1992, Trends of sandstone porosity in the Anadarko basin, in Dyman, T.S., ed., Geologic controls and resource potential of natural gas in deep sedimentary basins: U.S. Geological Survey Open-File Report 92-524, p. 63-75.

Hester, T.C., [submitted], Porosity trends of Pennsylvanian sandstones with respect to thermal maturity and thermal regimes in the Anadarko basin, Oklahoma: U.S. Geological Survey Bulletin, 40 p.

Schmoker, J.W., 1992, Distribution of porosity in sedimentary rocks as a function of time-temperature exposure, in Dyman, T.S., ed., Geologic controls and resource potential of natural gas in deep sedimentary basins: U.S. Geological Survey Open-File Report 92-524, p. 29-62.

Schmoker, J.W., and Gautier, D.L., 1988, Sandstone porosity as a function of thermal maturity: Geology, v. 16, no. 11, p. 1007-1010.

Schmoker, J.W., and Gautier, D.L., 1989, Compaction of basin sediments: Modeling based on time-temperature history: Journal of Geophysical Research, v. 94(B), no. 6, p. 7379-7386.

Schmoker, J.W., and Hester, T.C., 1990, Regional trends of sandstone porosity versus vitrinite reflectance--a preliminary framework, in Nuccio, V.F., and Barker, C.E., eds., Applications of thermal maturity studies to energy exploration: Rocky Mountain Section-SEPM, Denver, p. 53-60.

Schmoker, J.W., and Higley, D.K., 1991, Porosity trends of the Lower Cretaceous J sandstone, Denver basin, Colorado: Journal of Sedimentary Petrology, v. 61, no. 6, p. 909-920.

Schmoker, J.W., Nuccio, V.F., and Pitman, J.K., 1992, Porosity trends in predominantly nonmarine sandstones of the Upper Cretaceous Mesaverde Group, Uinta and Piceance basins, Utah and Colorado, in Fouch, T.D., Nuccio, V.F., and Chidsey, T.C., eds., Hydrocarbon and mineral resources of the Uinta basin, Utah and Colorado: Utah Geological Association Guidebook 20, p. 111-121. 